\title{
Intramolecular Cyclobutadiene Cycloaddition/Cyclopropanation/Thermal Fragmentation: An Effective Strategy for the Asymmetric Synthesis of Pleocarpenene and Pleocarpenone
}

\author{
Michael J. Williams, Holly L. Deak, and Marc L. Snapper \\ Department of Chemistry, Eugene F. Merkert Chemistry Center, Boston College, \\ 2609 Beacon Street, Chestnut Hill, MA 02467-3860. \\ marc.snapper@bc.edu \\ Supporting Information
}

General Information

TABLE OF CONTENTS

Experimental Procedures

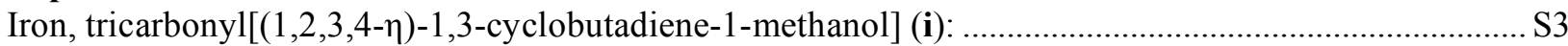

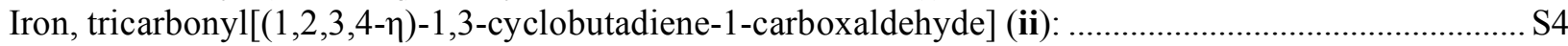

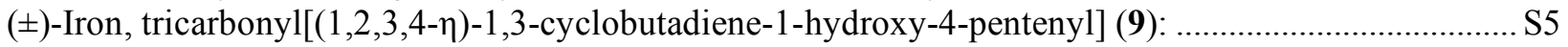

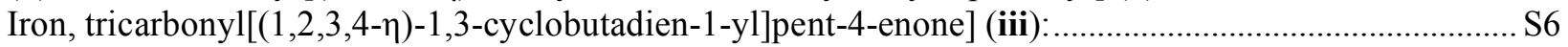

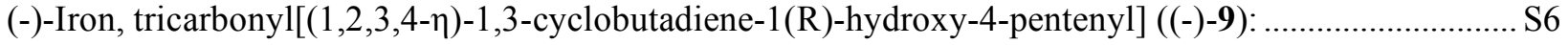

(-)-Iron, tricarbonyl [(1,2,3,4- $\eta)-1,3$-cyclobutadiene-1(R)-hydroxy-5-carbomethyoxy-4-(E)-pentenyl]

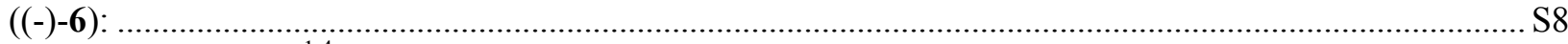

(-)-Tricyclo $\left[4 \cdot 3 \cdot 0.0^{1,4}\right]$ non-2-ene-5-carboxylic acid, 9-hydroxy-, methyl ester, $(1 S, 4 S, 5 S, 6 S, 9 R)((-)-5 \boldsymbol{\beta})$ :

(+)-(Tricyclo $\left[4.3 \cdot 0.0^{1,4}\right]$ non-2-ene-5-carboxylic acid, 9-hydroxy-, methyl ester, $(1 R, 4 R, 5 R, 6 R, 9 R)$

$((+)-5 \boldsymbol{\alpha})$ :

Compound (S)-(-)-MTPA-5及.

S14

Compound (R)-(-)-MTPA-5 $\boldsymbol{\beta}$

S15

Compound ((-)-13及)

Compound $((+)-\mathbf{1 3 \alpha})$

Compound $((-)-\mathbf{1 4 \beta})$

Compound $((+)-14 \alpha)$

Compound ((-)-4).

$\mathrm{S} 21$

Compound ((+)-4)

(-)-(1S,3a R,4R,5Z,7S,8E)-7-(2-hydroxypropan-2-yl)-1-methyl-4-((triisopropylsilyloxy)methyl)-

1,2,3,3a,4,7-hexahydroazulen-1-ol ((-)-3)

(+)-(1S,3a $R, 4 R, 7 S, 8 \mathrm{a} S)-7-(2-h y d r o x y p r o p a n-2-y l)-1-m e t h y l-4-(($ triisopropylsilyloxy)methyl)decahydroazulen-1-ol $((+)-15)$

(+)-(1S,3a R,4R,7S,8aS)-4-(hydroxymethyl)-7-(2-hydroxypropan-2-yl)-1-methyldecahydroazulen-1-ol

$((+)-\mathbf{i v})$

(+)-((1S,3a $R, 4 R, 7 S, 8 \mathrm{a} S)-1$-hydroxy-7-(2-hydroxypropan-2-yl)-1-methyldecahydroazulen-4-yl)methyl

4-methylbenzenesulfonate $((+)-\mathbf{v})$

(+)-5-Azulenemethanol, decahydro-3-hydroxy- $\alpha, \alpha, 3$-trimethyl-8-methylene-, $(3 S, 3 \mathrm{a} S, 5 S, 8 \mathrm{a} S)$ - $(9 \mathrm{Cl})$ (ent-

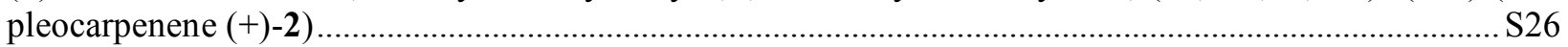

(-)-4(1H)-Azulenone, octahydro-1-hydroxy-7-(1-hydroxy-1-methylethyl)-1-methyl-(1S,3a $R, 7 S, 8 \mathrm{a} S)(9 \mathrm{Cl})$

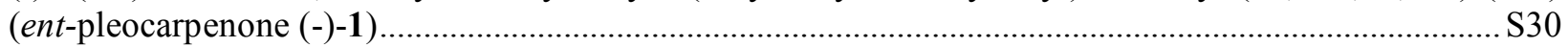

$(+)-4(1 \mathrm{H})$-Azulenone, $\quad$ octahydro-1-hydroxy-7-(1-hydroxy-1-methylethyl)-1-methyl-(1R,3a $S, 7 R, 8 \mathrm{a} R)$

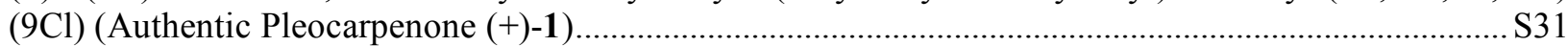

NMR \& IR spectra

S33-S85 


\section{General Information:}

Proton nuclear magnetic resonance spectra $\left({ }^{1} \mathbf{H}-\mathbf{N M R}\right)$ were measured on either a Gemini-400 instrument (400 MHz) or a Gemini-500 instrument (500 MHz). Chemical shifts are reported in ppm downfield from tetramethylsilane with the solvent reference as the internal standard $\left(\mathrm{CHCl}_{3}: \delta 7.26\right.$ ppm; $\left.\mathrm{CD}_{3} \mathrm{OD}: \delta 4.84 \mathrm{ppm}\right)$. Data is reported as follows: chemical shift, multiplicity ( $\mathrm{s}=$ singlet, $\mathrm{d}=$ doublet, $\mathrm{t}=$ triplet, $\mathrm{q}=$ quartet, $\mathrm{br}=$ broad, $\mathrm{m}=$ multiplet $)$, coupling constants $(\mathrm{Hz})$, and integration. ${ }^{13} \mathbf{C}-\mathbf{N M R}$ spectra were recorded on either a Gemini-400 instrument (100 MHz), or a Gemini-500 instrument (125 MHz) with complete proton decoupling. Chemical shifts are reported in ppm downfield from tetramethylsilane with the solvent as the internal reference $\left(\mathrm{CDCl}_{3}: \delta 77.23 \mathrm{ppm} ; \mathrm{CD}_{3} \mathrm{OD}: \delta 49.15 \mathrm{ppm}\right)$. Infrared spectra (IR) were reported in wave numbers $\left(\mathrm{cm}^{-1}\right)$. Bands are characterized as broad (br), strong (s), medium (m), or weak (w). Elemental analyses (Anal) were performed by Robertson Microlit Laboratories, Inc., Madison, NJ and are reported in percent atomic abundance. High resolution mass spectral analyses (HRMS) were performed by the Mass Spectrometry Laboratory, University of Illinois at Urbana-Champaign or at Boston College. Melting points (mp) were reported uncorrected.

Enantiomer ratios were determined by chiral GC analysis [Chiraldex GTA column (30 m x $0.25 \mathrm{~mm}$ ) and Betadex 120 column $(30 \mathrm{~m} \mathrm{x} 0.25 \mathrm{~mm}$ ], and by chiral HPLC analysis [Chiral pak AS column (250 mm x $4.6 \mathrm{~mm})$ and Chiral pak OD column (250 mm x $4.6 \mathrm{~mm})]$. All details associated with each separation are included with each compound. Optical rotations were measured on a polarimeter using the sodium D line $(\gamma=589)$ at the corrected temperature of 20 ${ }^{\circ} \mathrm{C}$ and are reported as follows: $[\alpha]^{\text {temp }}{ }_{\mathrm{D}}$, (concentration $(c=\mathrm{g} / 100 \mathrm{~mL})$, and solvent).

Starting materials and reagents were purchased from commercial suppliers and used without further purification except the following: Dichloromethane, DMF, hexane, toluene, diethyl ether, THF, and benzene were used from a solvent purification system. Hexanes and $\mathrm{Et}_{2} \mathrm{O}$ used in chromatography were distilled before use. Molecular sieves were dried in a $250{ }^{\circ} \mathrm{C}$ oven overnight before use. [Please note potential hazards: Iron carbonyls complexes can be a source of carbon monoxide. Diriron nonacarbonyl is a flammable solid].

All oxygen- or moisture-sensitive reactions were carried out under $\mathrm{N}_{2}$ or $\mathrm{Ar}$ atmosphere in oven-dried $\left(140{ }^{\circ} \mathrm{C}, \geq 4 \mathrm{~h}\right)$ or flame-dried glassware. Air- or moisture-sensitive liquids were transferred by syringe or cannula and were introduced into the reaction flasks through rubber 
septa or through a stopcock under $\mathrm{N}_{2}$ positive pressure. Degassing refers to a flow of dry $\mathrm{N}_{2(\mathrm{~g})}$ bubbling through reaction solvent for $15 \mathrm{~min}$. Unless otherwise stated, reactions were stirred with a Teflon covered stir bar. Concentration refers to the removal of solvent using a rotary evaporator followed by use of a vacuum pump at approximately 1 torr. Silica gel column chromatography refers to flash chromatography ${ }^{1}$ and was performed using $60 \AA$ (230-400 Mesh ASTM) silica gel. Percent (\%) Ag refers to silver nitrate treated silica gel and is measured as $\mathrm{wt} / \mathrm{wt}, \mathrm{AgNO}_{3} / \mathrm{SiO}_{2}{ }^{2}$ Thin layer chromatography was performed on glass back $60 \AA(250 \mu \mathrm{m}$ thickness) silica gel plates.

Experimental Procedures:

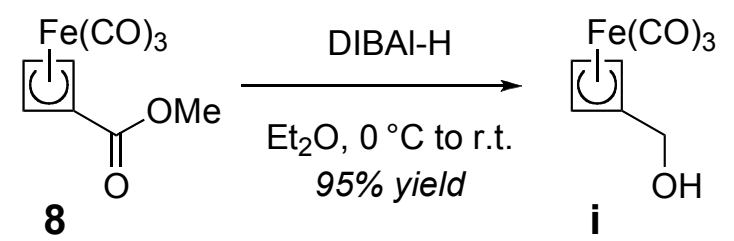

Iron, tricarbonyl[(1,2,3,4- $\eta$ )-1,3-cyclobutadiene-1-methanol] (i): To a solution of iron ester $\mathbf{8}^{3}(8.17 \mathrm{~g}, 32.7 \mathrm{mmol})$ stirring in $\operatorname{dry~}_{2} \mathrm{O}(65 \mathrm{~mL}, 0.5 \mathrm{M})$ at $0{ }^{\circ} \mathrm{C}$ was added neat diisobutylaluminum hydride (12.8 mL, $71.9 \mathrm{mmol}, 2.2$ equiv) dropwise. Upon complete addition, the solution was warmed to room temperature and stirred $(45 \mathrm{~min})$ until the reaction was judged complete by TLC analysis (3:1 hexanes: $\mathrm{Et}_{2} \mathrm{O}$ ) [Note: The solution went from a yellow-orange color to a clear and blackish solution upon addition of the diisobutylaluminum hydride]. The reaction was cooled to $0{ }^{\circ} \mathrm{C}$, diluted with $\mathrm{Et}_{2} \mathrm{O}(200 \mathrm{~mL})$, and carefully [note: out gassing] quenched with the addition of an aqueous saturated solution of sodium potassium tartrate (150 $\mathrm{mL}$ ). Upon addition, the solution became a thick, light tan gel that eventually turned biphasic with stirring $(2 \mathrm{~h})$. The solution was transferred to a separatory funnel with $\mathrm{Et}_{2} \mathrm{O}(50 \mathrm{~mL})$ and the organic phase was removed. The aqueous phase was extracted with $\mathrm{Et}_{2} \mathrm{O}(3 \mathrm{x} 50 \mathrm{~mL})$. The combined organic extracts were washed with brine $(50 \mathrm{~mL})$, dried over $\mathrm{MgSO}_{4}$, filtered, and concentrated. The resulting light red oil was passed through a plug of silica gel $(\sim 5 \mathrm{~cm})$ with $\mathrm{Et}_{2} \mathrm{O}$, and the eluant was collected and concentrated. The red oil was then treated with a solution of $\mathrm{Et}_{2} \mathrm{O}$ :hexanes $(10: 90,100 \mathrm{~mL})$ and concentrated until the volume was $\sim 25 \mathrm{~mL}$ at which point

\footnotetext{
${ }^{1}$ Stille, W.C.; Kahn, M.; Mitra, A. J. Org. Chem. 1978, 43, 2923-2925.

2 Rabel, F. Chrom. News Vol. 4, No. 1, 1995; EM Separations Technology, Gibbstown, NJ.

${ }^{3}$ Limanto, J.; Snapper, M. L. J. Am. Chem. Soc. 2000, 122, 8071-8072.
} 
a yellow precipitate formed. Additional hexanes $(50 \mathrm{~mL})$ was added to the flask and concentrated until the volume was $\sim 25 \mathrm{~mL}$. The suspension was then filtered through a Büchner funnel. The resulting filtrate formed a precipitate, and this was again filtered through the same Büchner filter to yield compound $\mathbf{i}(6.33 \mathrm{~g}, 28.5 \mathrm{mmol}, 87 \%$ yield $)$ as a flocculent yellow solid (mp $41-43{ }^{\circ} \mathrm{C}$ ). The filtrate was concentrated and further purified by gradient silica gel chromatography (20:80 $\mathrm{Et}_{2} \mathrm{O}$ :hexanes with a gradient to 40:60 $\mathrm{Et}_{2} \mathrm{O}$ :hexanes) yielding a second lot of compound $\mathbf{i}(0.56 \mathrm{~g}, 2.5 \mathrm{mmol}, 8 \%)$ as a yellow solid ( $\left.\mathbf{m p ~} 41-43{ }^{\circ} \mathrm{C}\right)$. ${ }^{1} \mathbf{H}-\mathbf{N M R}(400$ $\left.\mathrm{MHz}, \mathrm{CDCl}_{3}\right): \delta 4.15$ (s, 2H), $4.12(\mathrm{~s}, 1 \mathrm{H}), 4.00(\mathrm{~d}, J=6.2 \mathrm{~Hz}, 2 \mathrm{H}), 1.46(\mathrm{t}, J=6.2 \mathrm{~Hz}, 1 \mathrm{H})$. ${ }^{13}$ C-NMR (100 MHz, $\left.\mathrm{CDCl}_{3}\right): \delta 214.0,84.3,64.0,62.7,58.7$. IR (NaCl, thin film): 3371 (br), 2929 (w), 2874 (w), 2048 (s), 1955 (s), 1639 (m), 1449 (w), 1297 (w), 1074 (m), 998 (m), 614 (m), $589(\mathrm{~m}) \mathrm{cm}^{-1}$. Anal: Calc'd for $\mathrm{C}_{8} \mathrm{H}_{6} \mathrm{FeO}_{4}: \mathrm{C}, 43.29 ; \mathrm{H}, 2.72$. Found: C, 43.51; H, 2.86 .

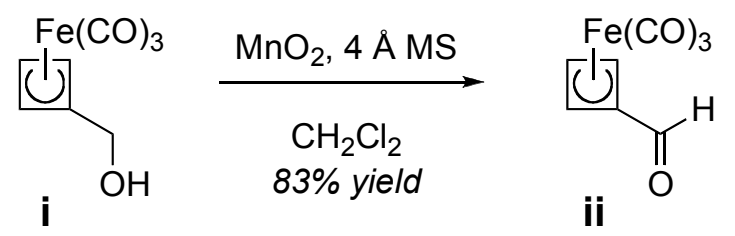

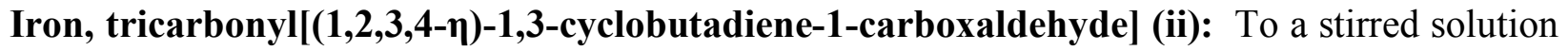
of iron alcohol $\mathbf{i}(3.00 \mathrm{~g}, 13.5 \mathrm{mmol})$ and powdered $4 \AA$ molecular sieves $(0.6 \mathrm{~g}, 20 \mathrm{wt} \%)$ in $\mathrm{CH}_{2} \mathrm{Cl}_{2}(27 \mathrm{~mL}, 0.5 \mathrm{M})$ was added $\mathrm{MnO}_{2}(11.7 \mathrm{~g}, 135 \mathrm{mmol}, 10$ equiv). The solution was allowed to stir $(10 \mathrm{~h})$ at which point the reaction was judged complete by TLC analysis (2:1 hexanes: $\left.\mathrm{Et}_{2} \mathrm{O}\right)$. The solution was filtered through Celite, washed with $\mathrm{CH}_{2} \mathrm{Cl}_{2}(\sim 200 \mathrm{~mL})$, and the filtrate concentrated. The resulting red oil was purified by gradient silica gel chromatography (10:90 $\mathrm{Et}_{2} \mathrm{O}$ :hexanes with a gradient to $30: 70 \mathrm{Et}_{2} \mathrm{O}$ :hexanes) to yield the desired aldehyde ii $(2.45 \mathrm{~g}, 11.1 \mathrm{mmol}, 83 \%$ yield) as a red oil that solidified to a yellow-red solid upon standing in the freezer $\left(\sim-20^{\circ} \mathrm{C}\right) .{ }^{1} \mathrm{H}-\mathrm{NMR}\left(400 \mathrm{MHz}, \mathrm{CDCl}_{3}\right): \delta 9.20(\mathrm{~s}, 1 \mathrm{H}), 4.65(\mathrm{~s}, 2 \mathrm{H}), 5.52(\mathrm{~s}, 1 \mathrm{H})$. ${ }^{13}$ C-NMR (100 MHz, $\left.\mathrm{CDCl}_{3}\right): \delta 211.1,187.5,71.8,69.8$, 65.3. IR (NaCl, thin film): $3106(\mathrm{w})$, 3852 (w), 2787 (w), 2728 (w), 2057 (s), 1973 (s), 1667 (s), 1432 (w), 1377 (w), 1302 (w), 1121 (w), $1058(w), 928(w), 828(w), 609$ (m), $584(\mathrm{~m}) \mathrm{cm}^{-1}$. Anal: Calc'd for $\mathrm{C}_{8} \mathrm{H}_{6} \mathrm{FeO}_{4}$ : C, 43.29; H, 1.83. Found: C, 43.64; H, 1.79 . 


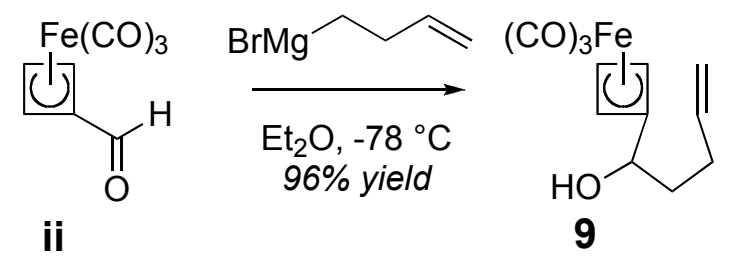

( \pm )-Iron, tricarbonyl[(1,2,3,4- $)$-1,3-cyclobutadiene-1-hydroxy-4-pentenyl] (9): [Note: The 3-butenyl magnesium bromide reagent was generated by the following procedure. To a flamedried two-neck $500 \mathrm{~mL}$ round bottom flask fitted with a septum and reflux condenser under a nitrogen atmosphere was added powdered magnesium $(6.1 \mathrm{~g}, 250 \mathrm{mmol}, 2.5$ equiv). Dry diethyl ether $(200 \mathrm{~mL}, 0.5 \mathrm{M})$ was added to the flask, and 4-bromobutene $(10.2 \mathrm{~mL}, 100 \mathrm{mmol}, 1.0$ equiv) was added to the reactor at rate of $10 \mathrm{~mL} / \mathrm{hr}$ with a syringe pump. The solution refluxed gently during addition and was allowed to stir at room temperature $(24 \mathrm{~h})$ at which time was titrated and found to be $0.41 \mathrm{M}(82 \%$ yield $)$.]

Compound ii (4.77 g, $21.7 \mathrm{mmol}, 1.0$ equiv) was added to a flame-dried $500 \mathrm{~mL}$ round bottom flask. Dry diethyl ether $(109 \mathrm{~mL}, 0.2 \mathrm{M})$ was added to the flask by syringe and the solution was cooled to $-78{ }^{\circ} \mathrm{C}$ (dry ice/acetone bath). Freshly made 3-butenyl magnesium bromide (63.4 mL, 0.41 M solution, $26.0 \mathrm{mmol}, 1.2$ equiv) (vide supra) was added dropwise to the stirred solution over a period of $\sim 10 \mathrm{~min}$. The reaction was allowed to stir at $-78{ }^{\circ} \mathrm{C}$, monitored by TLC analysis (4:1 hexanes:EtOAc), and was judged complete after $1 \mathrm{~h}$. The reaction was quenched at $-78{ }^{\circ} \mathrm{C}$ by dropwise addition of saturated aqueous $\mathrm{NH}_{4} \mathrm{Cl}(20 \mathrm{~mL})$, removed from the $-78{ }^{\circ} \mathrm{C}$ bath, and allowed to warm to room temperature. Additional saturated aqueous $\mathrm{NH}_{4} \mathrm{Cl}(80 \mathrm{~mL})$ was added to the biphasic solution, the layers were separated, and the aqueous layer was extracted with $\mathrm{Et}_{2} \mathrm{O}(3 \times 50 \mathrm{~mL})$. The combined organic extracts were washed with brine $(50 \mathrm{~mL})$, dried over $\mathrm{MgSO}_{4}$, filtered, and concentrated. The resulting red oil was purified by gradient silica gel chromatography (5:95 $\mathrm{Et}_{2} \mathrm{O}$ :hexanes with a gradient to 30:70 $\mathrm{Et}_{2} \mathrm{O}$ :hexanes) to afford compound 9 (5.77 g, $20.9 \mathrm{mmol}, 96 \%$ yield) as a reddish oil. ${ }^{1} \mathbf{H}-\mathbf{N M R}$ $\left(400 \mathrm{MHz}, \mathrm{CDCl}_{3}\right): \delta 5.82(\mathrm{ddt}, J=16.9,10.2,6.6 \mathrm{~Hz}, 1 \mathrm{H}), 5.07(\mathrm{dq}, J=17.2,1.6 \mathrm{~Hz}, 1 \mathrm{H}), 5.00$ $(\mathrm{dq}, J=10.2,1.7 \mathrm{~Hz}, 1 \mathrm{H}), 4.15(\mathrm{~s}, 1 \mathrm{H}), 4.13-4.03(\mathrm{~m}, 3 \mathrm{H}), 2.30-2.13(\mathrm{~m}, 2 \mathrm{H}), 1.64-1.59$ (m, 2H), 1.59 (d, $J=5.2 \mathrm{~Hz}, 1 \mathrm{H}) .{ }^{13} \mathbf{C}-\mathbf{N M R}\left(100 \mathrm{MHz}, \mathrm{CDCl}_{3}\right): \delta 214.2,137.7,115.5,89.8,67.1$, 62.9, 62.6, 62.2, 36.7, 30.2. IR (NaCl, thin film): 3409 (br m), 3077 (w), 2981 (w), 2931 (w), 2847 (w), 2042 (s), 1967 (br s), 1640 (w), 1443 (w), 1305 (w), 1062 (w), 995 (w), 915 (m), 613 (s), 588 (s) $\mathrm{cm}^{-1}$. Anal: Calc'd for $\mathrm{C}_{12} \mathrm{H}_{12} \mathrm{FeO}_{4}$ : C, 52.21; H, 4.38. Found: C, 52.39; H, 4.02. 


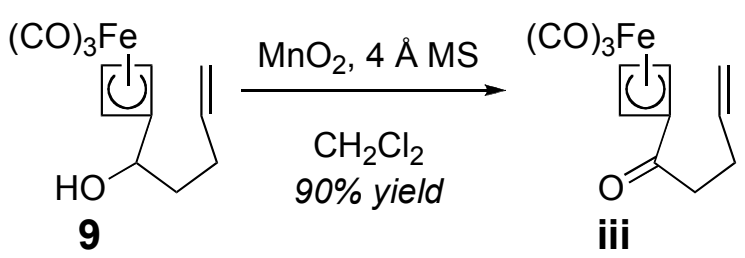

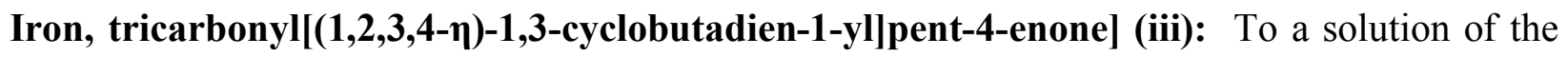
alcohol 9 (2.00 g, $7.24 \mathrm{mmol})$ stirring in dry $\mathrm{CH}_{2} \mathrm{Cl}_{2}(14.5 \mathrm{~mL}, 0.5 \mathrm{M})$ was added $4 \AA$ molecular sieves $(0.40 \mathrm{~g}, 20 \mathrm{wt} \%)$ and allowed to stir for $20 \mathrm{~min}$. After the allotted time, $\mathrm{MnO}_{2}(6.29 \mathrm{~g}$, $72.4 \mathrm{mmol}, 10.0$ equiv) was added to the reaction mixture and the black heterogeneous mixture was allowed to stir until the reaction was determined complete $(12 \mathrm{~h})$ by TLC analysis $(2: 1$ hexanes:Et $\left.{ }_{2} \mathrm{O}\right)$. The reaction was filtered through Celite with $\mathrm{CH}_{2} \mathrm{Cl}_{2}(\sim 200 \mathrm{~mL})$. The filtrate was concentrated, and the resulting red oil was purified by gradient silica gel chromatography (10:90 $\mathrm{Et}_{2} \mathrm{O}$ :hexanes with a gradient to $30: 70 \mathrm{Et}_{2} \mathrm{O}$ :hexanes) to afford the iron ketone iii (1.79 $\mathrm{g}$, $6.53 \mathrm{mmol}, 90 \%$ yield) as a red oil. ${ }^{1} \mathbf{H}-\mathbf{N M R}\left(400 \mathrm{MHz}, \mathrm{CDCl}_{3}\right): \delta 5.82-5.72(\mathrm{~m}, 1 \mathrm{H}), 5.03(\mathrm{~d}$, $J=18.5 \mathrm{~Hz}, 1 \mathrm{H}), 5.00(\mathrm{~d}, J=11.0 \mathrm{~Hz}, 1 \mathrm{H}), 4.55(\mathrm{~s}, 2 \mathrm{H}), 4.42(\mathrm{~s}, 1 \mathrm{H}), 2.33-2.26(\mathrm{~m}, 4 \mathrm{H}) .{ }^{13} \mathrm{C}-$ NMR (100 MHz, $\left.\mathrm{CDCl}_{3}\right): \delta 211.8,199.0,136.7,115.7,70.2,69.6,64.9,37.3,28.6$. IR $(\mathrm{NaCl}$, thin film): 3087 (w), 2978 (w), 2924 (w), 2057 (s), 1982 (br s), 1668 (s), 1434 (s), 1308 (w), 1128 (m), 1086 (w), 994 (w), 961 (w), 919 (m), 831 (w), 609 (s), 588 (s) cm ${ }^{-1}$. Anal: Calc'd for $\mathrm{C}_{12} \mathrm{H}_{10} \mathrm{FeO}_{4}$ : C, 52.59; H, 3.68. Found: C, 52.66; H, 3.53.

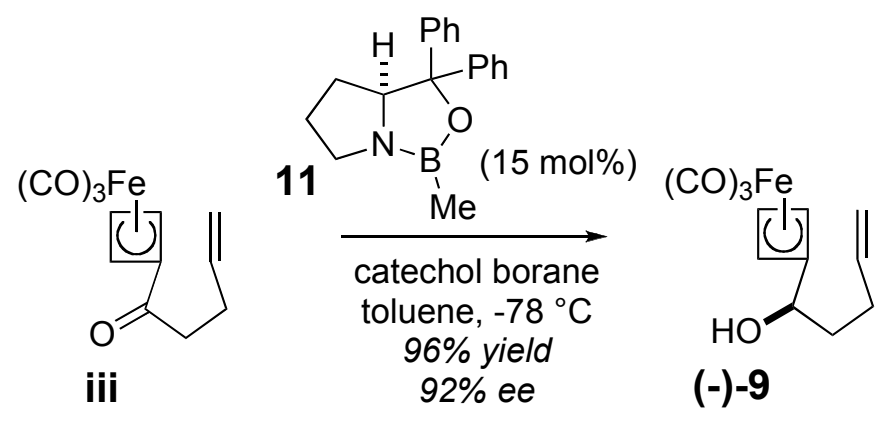

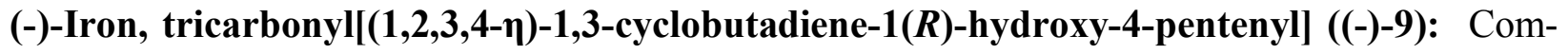
pound iii $(6.00 \mathrm{~g}, 21.9 \mathrm{mmol})$ was dried in dry toluene $(20 \mathrm{~mL}, 1.1 \mathrm{M})$ over $3 \AA$ molecular sieves $^{4}(6.0 \mathrm{~g}, 100 \mathrm{wt} \%)$ under a nitrogen atmosphere $(\sim 2 \mathrm{~h})$. The solution was then transferred using dry toluene $(64.3 \mathrm{~mL})$ to a dry $500 \mathrm{~mL}$ Schlenk flask fitted with a septum under a nitrogen atmosphere. A solution of $(S)$-B-Me-CBS catalyst (11) $(3.29 \mathrm{~mL}, 1 \mathrm{M}$ in toluene, $3.29 \mathrm{mmol}$,

\footnotetext{
${ }^{4}$ Jones, T. K.; Mohan, J. J.; Xavier, L. C.; Blacklock, T. J.; Mathre, D. J.; Sohar, P.; Turner Jones, E. T.; Reamer, R. A.; Roverts, F. E.; Grabowski, E. J. J. J. Org. Chem. 1991, 56, 763-769.
} 
0.15 equiv) was added to the reaction (final concentration $0.25 \mathrm{M}$ ), which was then cooled to $-78{ }^{\circ} \mathrm{C}$ and allowed to stir ( $\left.30 \mathrm{~min}\right)$. Catechol borane $(3.51 \mathrm{~mL}, 32.9 \mathrm{mmol}, 1.5 \mathrm{equiv})$ was added dropwise $(\sim 10 \mathrm{~min})$ to the flask. After the addition was complete, the reaction mixture was allowed to stir at $-78^{\circ} \mathrm{C}$ at which point $(12 \mathrm{~h})$ the reaction was judged complete by TLC analysis $\left(2: 1 \mathrm{Et}_{2} \mathrm{O}\right.$ :hexanes). The reaction was quenched at $-78{ }^{\circ} \mathrm{C}$ by the addition of $\mathrm{Et}_{2} \mathrm{O}$ $(150 \mathrm{~mL})$ and cautious, dropwise addition of $\mathrm{NaOH}(1 \mathrm{M}, 75 \mathrm{~mL}$ over $\sim 20 \mathrm{~min})$. The solution was removed from the $-78{ }^{\circ} \mathrm{C}$ bath and allowed to stir and warm to room temperature $(\sim 1 \mathrm{~h})$ with stirring. The resulting biphasic black solution was transferred with $\mathrm{Et}_{2} \mathrm{O}(50 \mathrm{~mL})$, extracted with $\mathrm{NaOH}(1 \mathrm{M}, 5 \times 100 \mathrm{~mL})$, water $(2 \times 50 \mathrm{~mL})$, brine $(50 \mathrm{~mL})$, dried over $\mathrm{MgSO}_{4}$, filtered, and concentrated to yield a yellow-brown oil. The oil was purified by gradient silica gel chromatography (10:90 $\mathrm{Et}_{2} \mathrm{O}$ :hexanes with a gradient to $30: 70 \mathrm{Et}_{2} \mathrm{O}$ :hexanes) to yield compound 9 (5.81 g, $21.1 \mathrm{mmol}, 96 \%$ yield, $92 \%$ ee $)$ as a yellow oil. $[\alpha]^{20}:-9.2^{\circ}\left(c=2.5, \mathrm{CHCl}_{3}\right)$. All spectra matched the racemic alcohol 9 vide supra.

(Chiral HPLC separation conditions: Chiral pak AS column (250 mm x $4.6 \mathrm{~mm}), 1 \mathrm{~mL} / \mathrm{min}, 99: 1$ (hexanes:IPA), $254 \mathrm{~nm}$ )

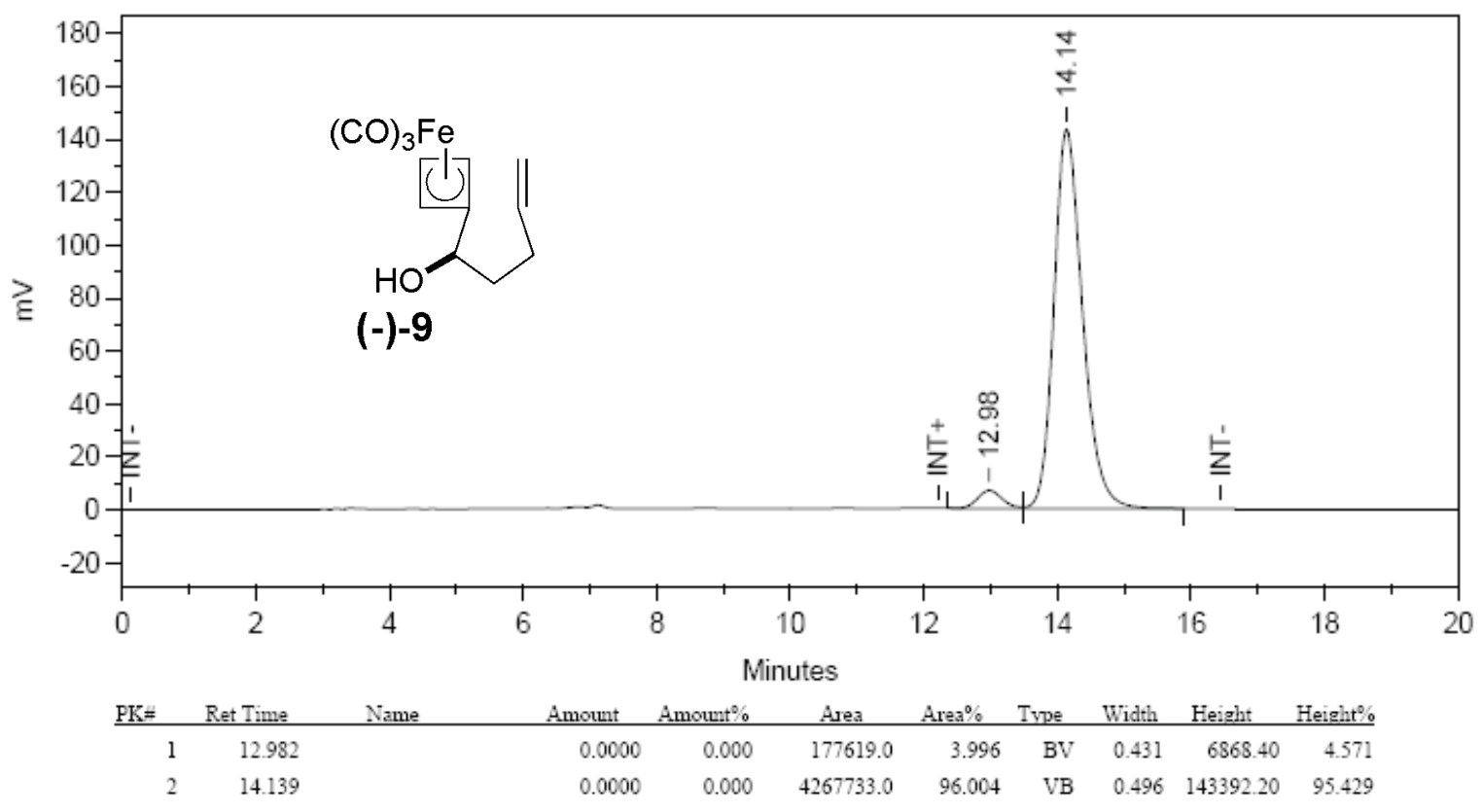

Total Area $=4445352.0$, Total Amount $=0.0$, Total Height $=150260.6$ 


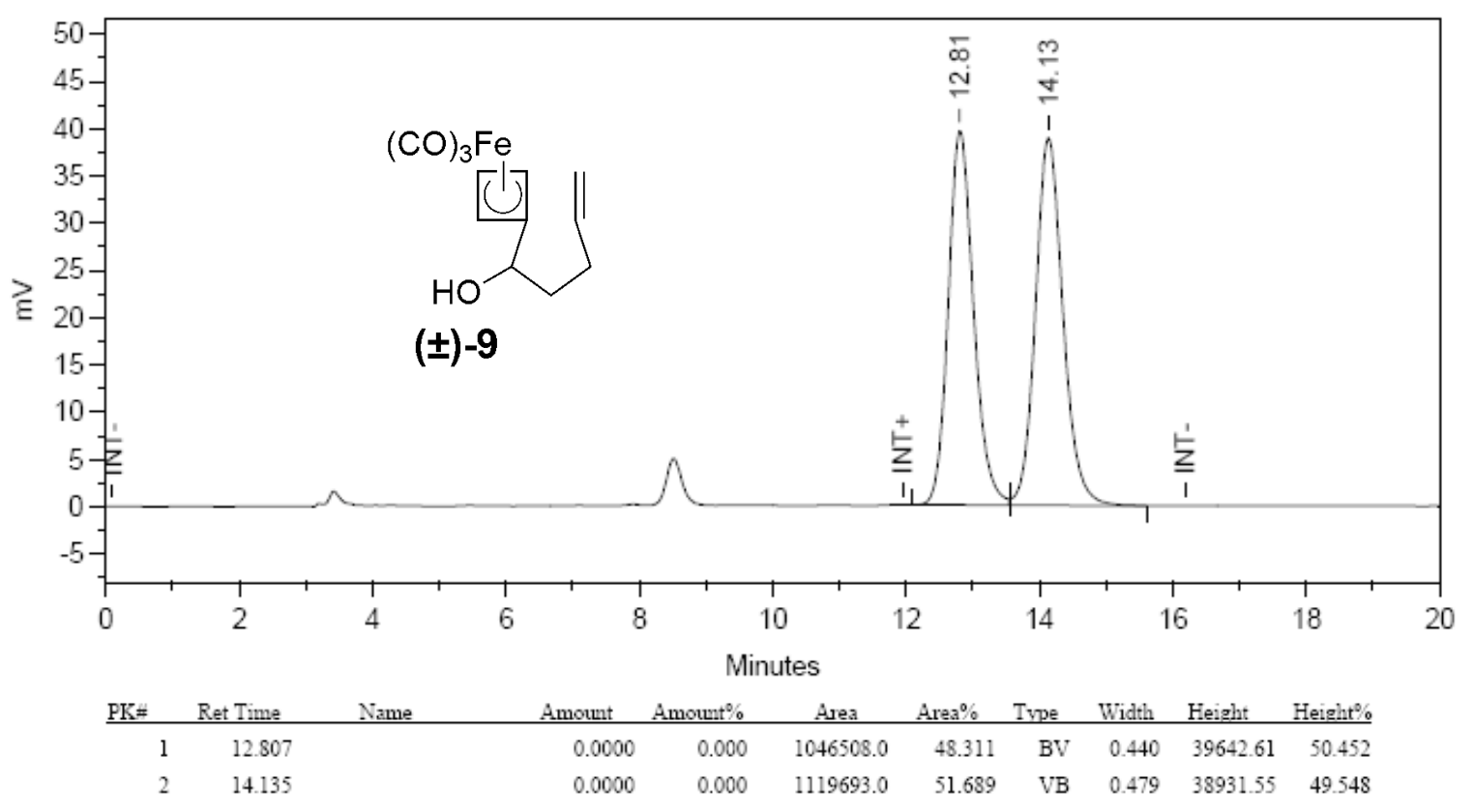

Total Area $=2166201.0$, Total Amourt $=0.0$, Total Height $=78574.16$

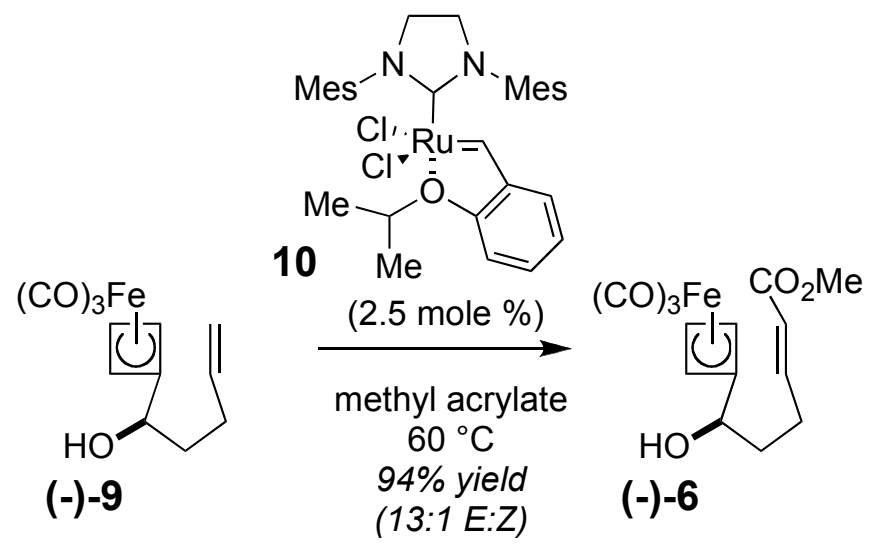

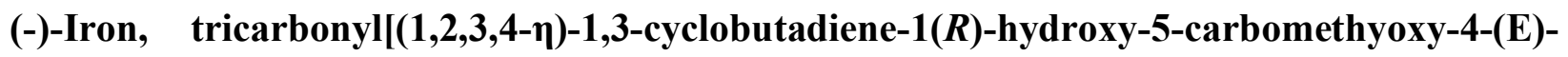
pentenyl] ((-)-6): In a $250 \mathrm{~mL}$ 2-neck round bottom flask was placed compound (-)-9 (5.45 g, $19.7 \mathrm{mmol})$, methyl acrylate $\left(17.0 \mathrm{~mL}, 197 \mathrm{mmol}, 10.0\right.$ equiv), and Hoveyda-Grubbs $2^{\text {nd }}$ generation catalyst $\mathbf{1 0}$ (308 mg, $0.491 \mathrm{mmol}, 0.025$ equiv). A reflux condenser was fitted to the neck, and the system was purged with $\mathrm{N}_{2}$ at which time the other neck was fitted with a septum. The reaction was then immersed into a $60{ }^{\circ} \mathrm{C}$ oil bath and stirred for $45 \mathrm{~min}$ [note: bubbling]. After the $45 \mathrm{~min}$ the reaction was checked by TLC analysis (30:70 EtOAc:hexanes) and judged complete. The reaction was removed from the bath and diluted with 10:90 EtOAc:hexanes (40 $\mathrm{mL})$. Silica gel (15.0 g, 300 wt\%) was added to the solution. The reaction was allowed to stir at room temperature (30 $\mathrm{min}$ ), and transferred to the top of a silica gel column (pre-wetted with 
10:90 EtOAc:hexanes). The silica gel column was flushed with 10:90 EtOAc:hexanes for several column volumes at which time the solvent ratio was gradually changed to 30:70 EtOAc:hexanes to afford compound (-)-6 (6.18 g, $18.5 \mathrm{mmol}, 94 \%$ yield with 13:1 E:Z ratio determined by NMR) as a red oil. $[\alpha]^{20}{ }_{\mathrm{D}}:-1.0^{\circ}\left(c=2.5, \mathrm{CHCl}_{3}\right) .{ }^{1} \mathbf{H}-\mathbf{N M R}\left(400 \mathrm{MHz}, \mathrm{CDCl}_{3}\right): \delta$ $6.96(\mathrm{dt}, J=15.6,7.2 \mathrm{~Hz}, 1 \mathrm{H}), 5.85(\mathrm{dt}, J=16.6,1.6 \mathrm{~Hz}, 1 \mathrm{H}), 4.16(\mathrm{~s}, 1 \mathrm{H}), 4.11(\mathrm{~d}, J=9.2 \mathrm{~Hz}$, $1 \mathrm{H}), 4.05(\mathrm{td}, J=8.0,5.2 \mathrm{~Hz}, 1 \mathrm{H}), 4.03(\mathrm{~d}, J=9.2 \mathrm{~Hz}, 1 \mathrm{H}), 3.72(\mathrm{~s}, 3 \mathrm{H}), 2.42$ (dqd, $J=13.6$, 6.8, 1.6 Hz, 1H), 2.33 (dqd, $J=15.6,6.8,1.2 \mathrm{~Hz}, 1 \mathrm{H}), 1.67$ (ddd, $J=11.6,4.8,2.8 \mathrm{~Hz}, 2 \mathrm{H}), 1.54$ $(\mathrm{d}, J=5.2 \mathrm{~Hz}, 1 \mathrm{H}) .{ }^{13} \mathrm{C}-\mathbf{N M R}\left(100 \mathrm{MHz}, \mathrm{CDCl}_{3}\right): \delta 214.3,167.1,148.3,121.8,89.2,66.8$, 62.8, 62.1, 51.7, 35.7, 28.6. IR (NaCl, thin film): 3446 (br), 2952 (w), 2046 (s), 1997 (s), 1721 (s), 1656 (m), 1438 (m), 1280 (m), 1206 (m), 1040 (m), 980 (w), 614 (s), 591 (s) cm . Anal: Calc'd for $\mathrm{C}_{14} \mathrm{H}_{14} \mathrm{FeO}_{6}$ : C, 50.33; H, 4.22. Found: C, 50.09; H, 4.44.

Chiral HPLC separation conditions: Chiral pak OD column (250 mm x $4.6 \mathrm{~mm}), 1 \mathrm{~mL} / \mathrm{min}$, 95:5 (hexanes:IPA), $254 \mathrm{~nm}$.

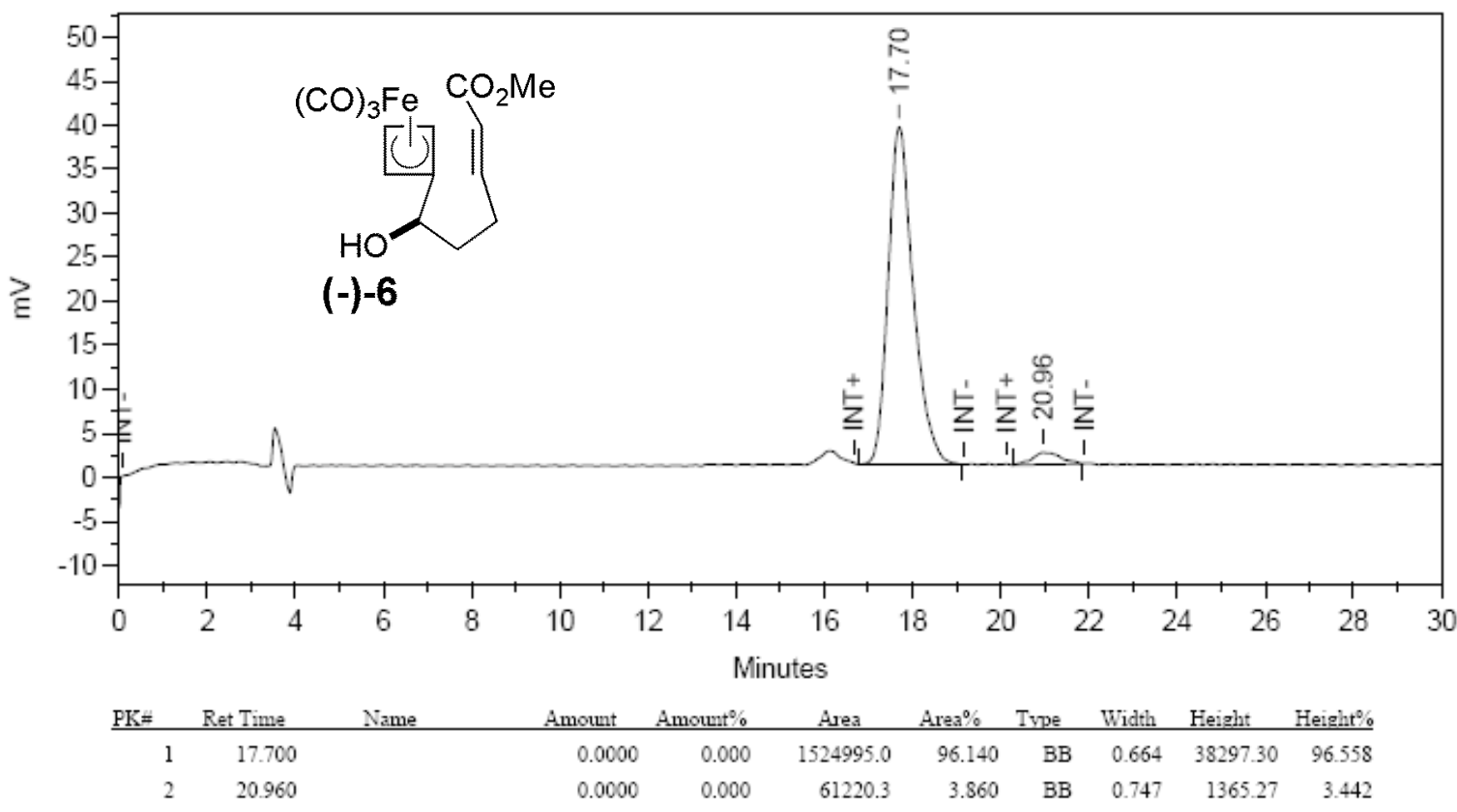

Total Area $=1586216.0$, Total Amount $=0.0$, Total Height $=39662.56$ 


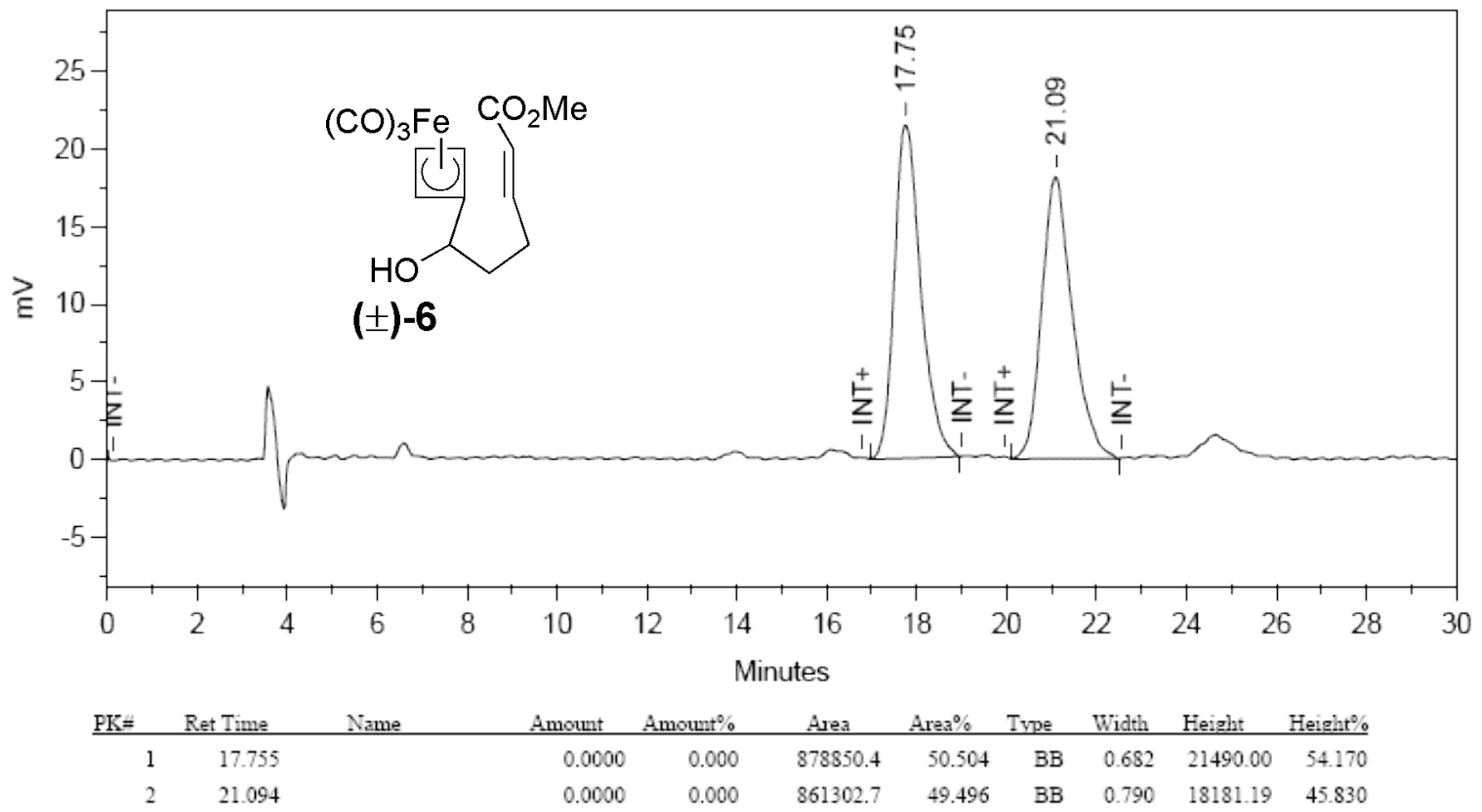

Total Area $=1740153.0$, Total Amourt $=0.0$, Total Height $=39671.19$

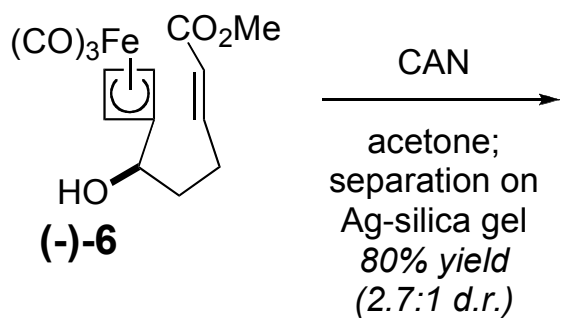

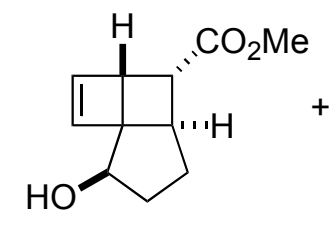

$(+)-5 \alpha$

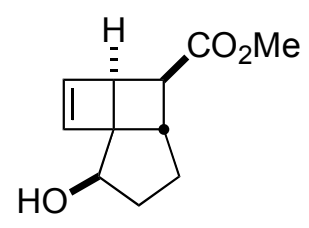

$(-)-5 \beta$

To a stirred solution of iron enone (-)-6 (6.18 g, $18.5 \mathrm{mmol})$ in acetone $(925 \mathrm{~mL}, 0.02 \mathrm{M})$ was added cerric ammonium nitrate $(30.4 \mathrm{~g}, 55.5 \mathrm{mmol}, 3.0$ equiv) [note: bubbling and solution becomes a deep red color]. After $30 \mathrm{~min}$, the reaction was judged complete by TLC analysis (2:1 $\mathrm{Et}_{2} \mathrm{O}$ :hexanes) and quenched by addition of saturated aqueous $\mathrm{NaHCO}_{3}(250 \mathrm{~mL})$ [note: a cloudy orange precipitate forms]. The solution was allowed to stir $(10 \mathrm{~min})$, stopped, and the precipitate was allowed to settle $(1 \mathrm{hr})$. The acetone was decanted from the precipitate, and the salt was then washed with acetone $(2 \times 200 \mathrm{~mL})$ and $\mathrm{CH}_{2} \mathrm{Cl}_{2}(3 \times 100 \mathrm{~mL})$ by decantation. The organic extracts were combined and concentrated until an orange aqueous layer remained. The aqueous layer was then extracted with $\mathrm{Et}_{2} \mathrm{O}(3 \times 50 \mathrm{~mL})$. The organic extracts were combined, washed with brine $(100 \mathrm{~mL})$, dried over $\mathrm{MgSO}_{4}$, filtered, and concentrated to yield a yelloworange oil. The oil was purified by Ag-impregnated gradient silica gel chromatography (10\% $\mathrm{Ag}, 9: 1 \mathrm{Et}_{2} \mathrm{O}$ :hexanes with a gradient to $100 \% \mathrm{Et}_{2} \mathrm{O}$ then $100 \% \mathrm{EtOAc}$ ) to yield two separate 
diastereomers (-)-5 $\boldsymbol{\beta}(2.10 \mathrm{~g}, 10.8 \mathrm{mmol})$ and $(+)-5 \boldsymbol{\alpha}(0.76 \mathrm{~g}, 3.9 \mathrm{mmol})$ in a $2.7: 1 \mathrm{ratio}$ in $80 \%$ combined yield.

\section{(-) - Tricyclo $\left[4.3 .0 .0^{1,4}\right]$ non-2-ene-5-carboxylic acid, 9-hydroxy-, methyl}

ester, $\left(\mathbf{1 S}, \mathbf{4 S}, \mathbf{5 S}, \mathbf{6 S , 9 R )}((-)-5 \beta)\right.$ : Clear oil; $[\alpha]_{\mathrm{D}}^{20}:-210^{\circ}\left(c=2.0, \mathrm{CHCl}_{3}\right)$;

${ }^{1}$ H-NMR $\left(400 \mathrm{MHz}, \mathrm{CDCl}_{3}\right): \delta 6.49(\mathrm{dd}, J=2.4,1.6 \mathrm{~Hz}, 1 \mathrm{H}), 6.22(\mathrm{dd}, J=$

2.8, $0.4 \mathrm{~Hz}, 1 \mathrm{H}), 4.02(\mathrm{dd}, J=3.6,1.6 \mathrm{~Hz}, 1 \mathrm{H}), 3.65(\mathrm{~s}, 3 \mathrm{H}), 3.07(\mathrm{dd}, J=$

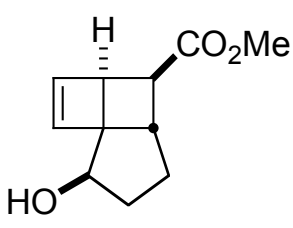

$(-)-5 \beta$

2.8, $0.4 \mathrm{~Hz}, 1 \mathrm{H}), 2.73(\mathrm{dd}, J=14.4,6.0 \mathrm{~Hz}, 2 \mathrm{H}), 2.16$ (tt, $J=12.8,8.0 \mathrm{~Hz}, 1 \mathrm{H}), 1.91(\mathrm{~m}, 2 \mathrm{H})$, $1.72(\mathrm{~m}, 1 \mathrm{H}), 1.59$ (br s, $1 \mathrm{H}){ }^{13} \mathbf{C}-\mathbf{N M R}\left(100 \mathrm{MHz}, \mathrm{CDCl}_{3}\right): \delta 173.9,139.8,136.9,74.3,62.6$, 51.7, 44.0, 41.9, 41.5, 34.3, 29.1. IR (NaCl, thin film): 3415 (br), 3091 (w), 3032 (w), 2949 (s), 1726 (s), 1539 (w), 1438 (m), 1245 (m), 1203 (m), 1174 (m), 1099 (m), 1015 (m), 969 (m), $798(\mathrm{w}), 743(\mathrm{~m}) \mathrm{cm}^{-1}$.

(Chiral GC separation conditions: Chiraldex GTA column (30 m x $0.25 \mathrm{~mm}$ ), 15 psi head pressure, $115^{\circ} \mathrm{C}$ isothermal, FID detector)

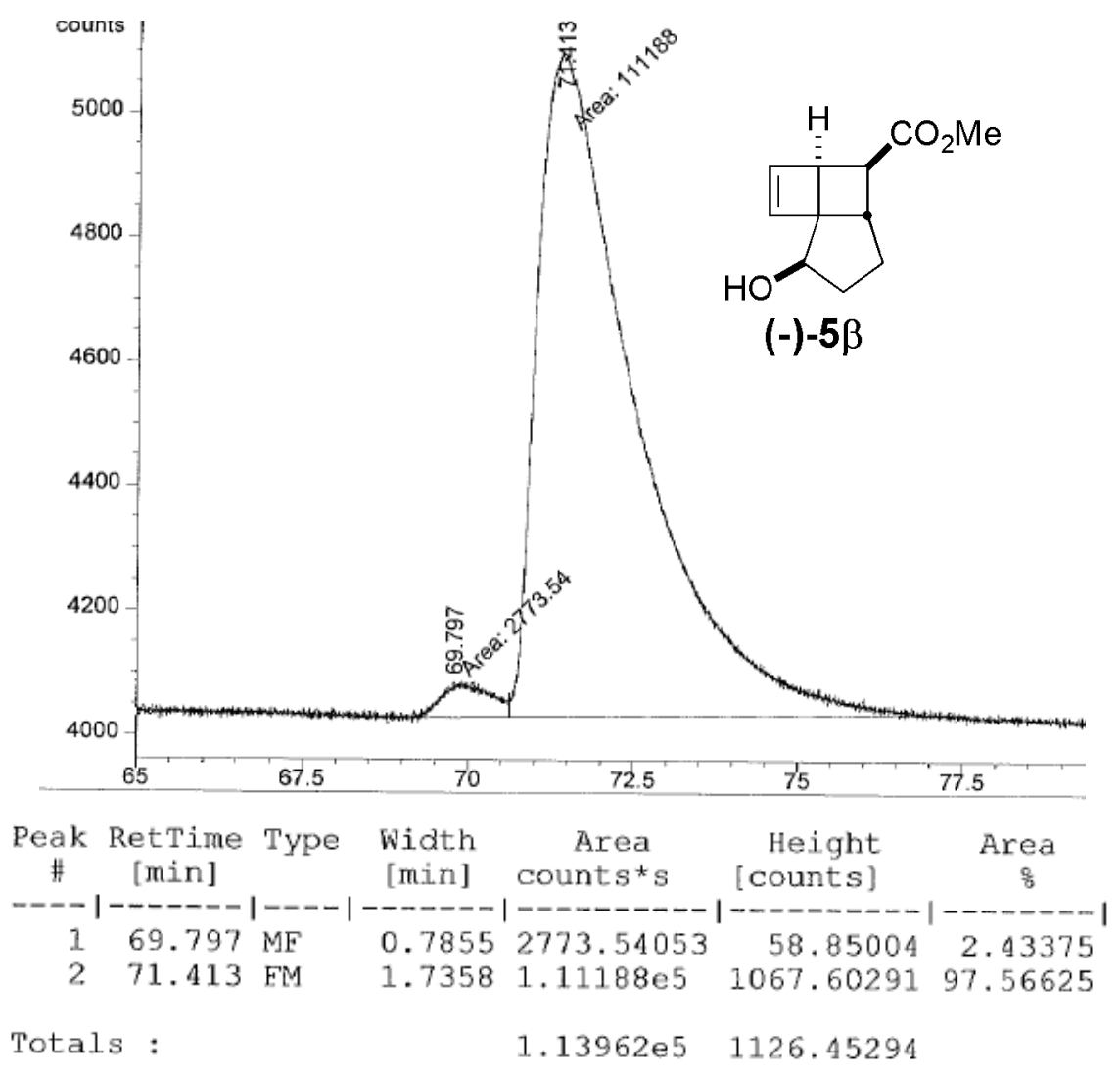




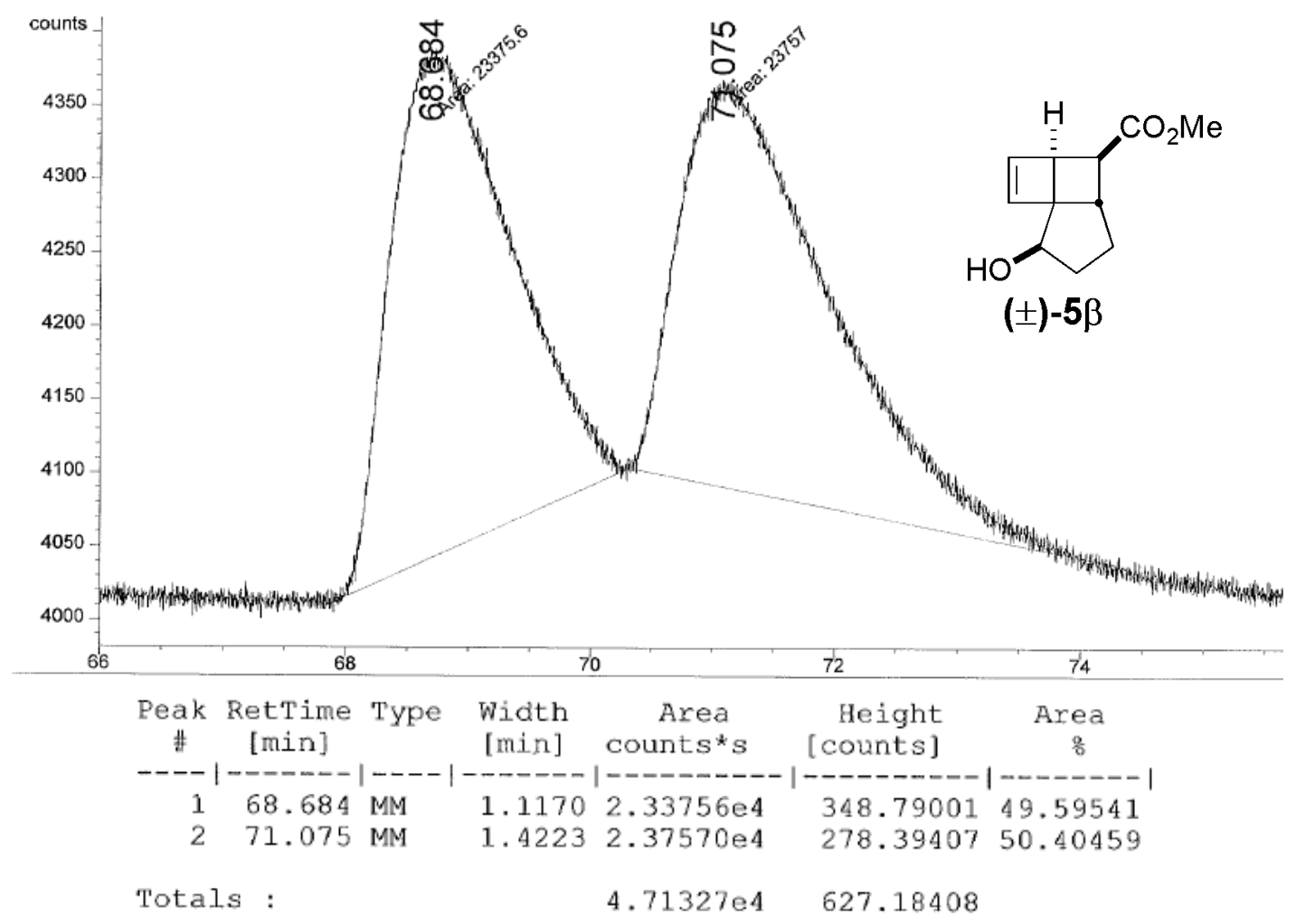

(+) - (Tricyclo[4.3.0.0 $\left.{ }^{1,4}\right]$ non-2-ene-5-carboxylic acid, 9-hydroxy-, methyl ester, $(1 \boldsymbol{R}, 4 \boldsymbol{R}, 5 \boldsymbol{R}, 6 \boldsymbol{R}, 9 \boldsymbol{R})((+)-5 \alpha)$ : Clear oil; $[\alpha]^{20}{ }_{\mathrm{D}}:+180^{\circ}\left(c=2.0, \mathrm{CHCl}_{3}\right)$; ${ }^{1}$ H-NMR $\left(400 \mathrm{MHz}, \mathrm{CDCl}_{3}\right): \delta 6.29(\mathrm{dd}, J=2.1,1.1 \mathrm{~Hz}, 1 \mathrm{H}), 6.22(\mathrm{~d}, J=$ $2.4 \mathrm{~Hz}, 1 \mathrm{H}), 4.27(\mathrm{dd}, J=9.6,6.3 \mathrm{~Hz}, 1 \mathrm{H}), 3.66(\mathrm{~s}, 3 \mathrm{H}), 3.42(\mathrm{~d}, J=8.7 \mathrm{~Hz}, \quad(+)-5 \alpha$ $1 \mathrm{H}), 2.79(\mathrm{dd}, J=8.7,4.8 \mathrm{~Hz}, 1 \mathrm{H}), 2.11(\mathrm{dt}, J=10.5,6.0 \mathrm{~Hz}, 1 \mathrm{H}), 1.86(\mathrm{dd}, J=15.3,7.8 \mathrm{~Hz}$, 1H) $1.80-1.67$ (m, 2H), 1.43 (br s, 1H). ${ }^{13} \mathbf{C}-\mathbf{N M R}\left(100 \mathrm{MHz}, \mathrm{CDCl}_{3}\right): \delta$ 173.8, 139.5, 138.0, 73.1, 62.0, 51.8, 42.5, 41.1, 40.9, 33.1, 27.7. IR ( NaCl, thin film): 3426 (br), $3091(\mathrm{w}), 3024$ (w), 2949 (s), 2861 (m), 1726 (s), 1433 (m), 1245 (s), 1195 (m), 1174 (m), 1103 (m), 1078 (m), $1019(\mathrm{~m}), 935(\mathrm{w}), 743(\mathrm{~m}) \mathrm{cm}^{-1}$. 
(Chiral GC separation conditions: Betadex 120 column (30 m x $0.25 \mathrm{~mm}$ ), 25 psi head pressure, $140{ }^{\circ} \mathrm{C}$ isothermal, FID detector)
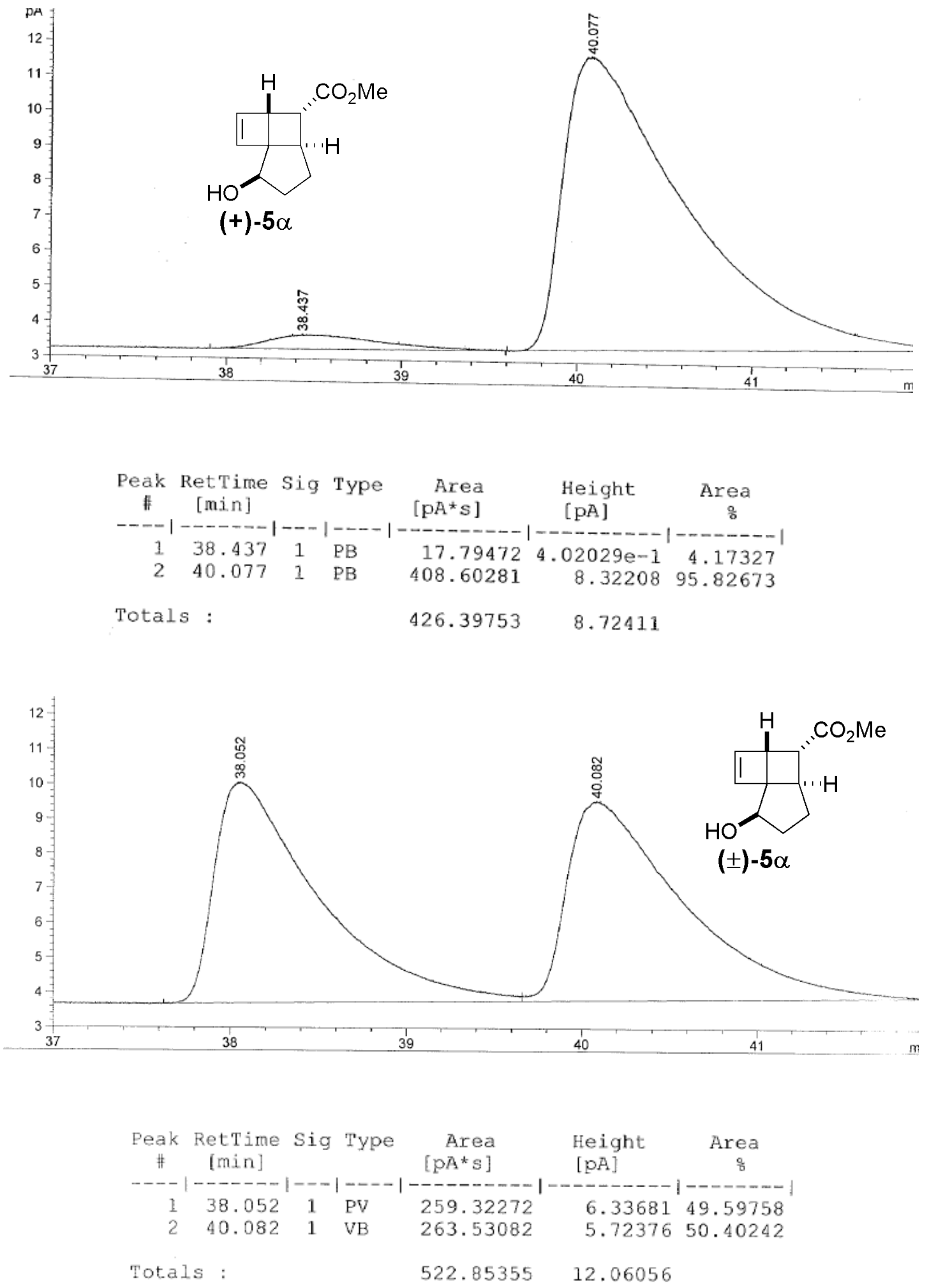


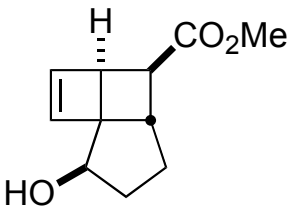

$(-)-5 \beta$

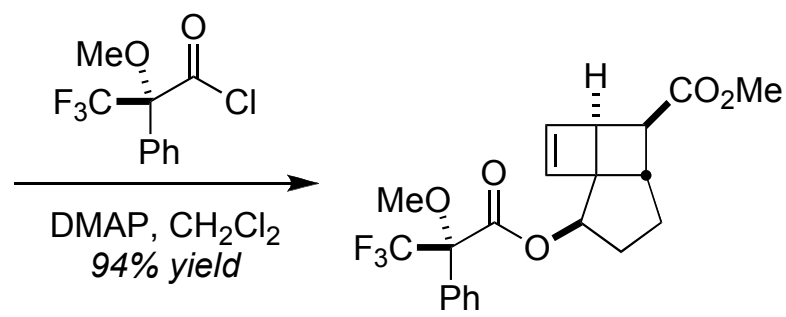

(S)-(-)-MTPA-5 $\beta$

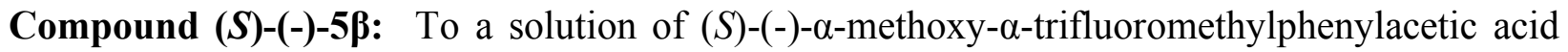
(51 mg, $0.22 \mathrm{mmol}, 2.0$ equiv) and dimethylformamide (17 $\mu \mathrm{L}, 0.22 \mathrm{mmol}, 2.0$ equiv) in dry hexanes $(2 \mathrm{~mL})$ was added freshly distilled oxalyl chloride $(29 \mu \mathrm{L}, 0.33 \mathrm{mmol}, 3.0$ equiv) and allowed to stir at room temperature under nitrogen $(2 \mathrm{~h})$. The hexanes layer of the biphasic mixture was removed, placed into another flask, and concentrated. The concentrate was dissolved in $\mathrm{CH}_{2} \mathrm{Cl}_{2}(0.5 \mathrm{~mL})$ and a solution of cyclobutene (-)-5及 (24 mg, $0.12 \mathrm{mmol}, 1.0$ equiv) and 4-dimethylaminopyridine ( $80 \mathrm{mg}, 0.65 \mathrm{mmol}, 5.3$ equiv) in $\mathrm{CH}_{2} \mathrm{Cl}_{2}(1.0 \mathrm{~mL}+0.5 \mathrm{~mL}$ wash) was added by syringe. The solution was allowed to stir overnight and judged complete by TLC analysis (1:1 hexanes:Et $\left.{ }_{2} \mathrm{O}\right)$. The reaction was diluted with $\mathrm{CH}_{2} \mathrm{Cl}_{2}(3 \mathrm{~mL})$ and washed with saturated $\mathrm{NaHCO}_{3}(2 \mathrm{~mL})$, water $(1 \mathrm{~mL})$, and brine $(2 \mathrm{~mL})$. The organic layer was dried over $\mathrm{MgSO}_{4}$, filtered, and concentrated. The product was purified by gradient silica gel chromatography (10:90 $\mathrm{Et}_{2} \mathrm{O}$ :hexanes with a gradient to 50:50 $\mathrm{Et}_{2} \mathrm{O}$ :hexanes) to afford (S)-(-)-MTPA-5 $\boldsymbol{\beta}$ (48 mg, $0.12 \mathrm{mmol}, 94 \%$ yield) as a clear, colorless oil. $[\alpha]^{20}{ }_{\mathrm{D}}:-130^{\circ}\left(c=2.0, \mathrm{CHCl}_{3}\right){ }^{1} \mathbf{H}-\mathbf{N M R}$ $\left(400 \mathrm{MHz}, \mathrm{CDCl}_{3}\right): \delta$ 7.53-7.51 (m, 2H), 7.42-7.39 (m, 3H), $6.18(\mathrm{dd}, J=2.5,0.5 \mathrm{~Hz}, 1 \mathrm{H}), 6.14$ $(\mathrm{dd}, J=2.4,1.5 \mathrm{~Hz}, 1 \mathrm{H}), 5.28-5.27(\mathrm{~m}, 1 \mathrm{H}), 3.65(\mathrm{~s}, 3 \mathrm{H}), 3.52(\mathrm{q}, J=1.1 \mathrm{~Hz}, 3 \mathrm{H}), 3.20(\mathrm{tdd}, J=$ 8.3, 1.4, 0.6 Hz, 1H), 2.77 (dd, $J=8.4,4.9 \mathrm{~Hz}, 1 \mathrm{H}), 2.72(\mathrm{dd}, J=7.7,4.7 \mathrm{~Hz}, 1 \mathrm{H}), 2.13-2.08$ (m, 2H), 2.09-1.99 (m, 1H), 1.83-1.77 (m, 1H). ${ }^{13}$ C-NMR (100 MHz, $\left.\mathrm{CDCl}_{3}\right): \delta$ 173.5, 166.0, $139.5,136.8,132.4,129.8,128.6,127.5,125.0,122.1,79.3,60.2,55.4,51.8,44.0,41.9,41.3$, 32.1, 29.3. IR (NaCl, thin film): 2948 (w), 1734 (s), 1439 (w), 1263 (m), 1242 (m), 1171 (s), 1009 (m), 867 (w), $717(\mathrm{~m}) \mathrm{cm}^{-1}$. ESI-HRMS (m/z): Calc'd for $\mathrm{C}_{21} \mathrm{H}_{21} \mathrm{~F}_{3} \mathrm{NaO}_{5}(\mathrm{M}+\mathrm{Na})$ : 433.1239; Found: 433.1230. 


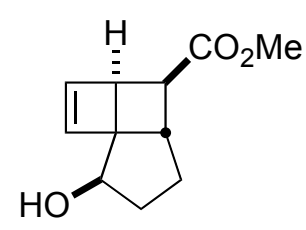

$(-)-5 \beta$

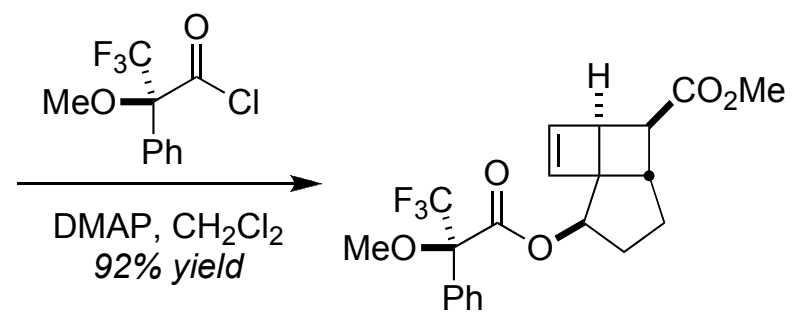

$(R)-(-)-M T P A-5 \beta$

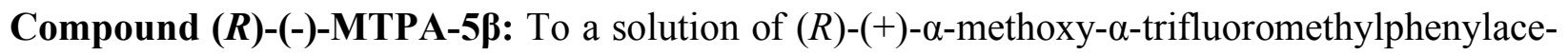
tic acid (57 mg, $0.24 \mathrm{mmol}, 1.6$ equiv) and dimethylformamide (18 $\mu \mathrm{L}, 0.24 \mathrm{mmol}, 1.6$ equiv) in dry hexanes $(2 \mathrm{~mL})$ was added freshly distilled oxalyl chloride $(30 \mu \mathrm{L}, 0.35 \mathrm{mmol}, 2.3$ equiv) and allowed to stir at room temperature under nitrogen $(2 \mathrm{~h})$. The hexanes layer of the biphasic mixture was removed, placed into another flask, and concentrated. The concentrate was dissolved in $\mathrm{CH}_{2} \mathrm{Cl}_{2}(0.5 \mathrm{~mL})$ and a solution of cyclobutene (-)-5及 (29 mg, $0.15 \mathrm{mmol}, 1.0$ equiv) and 4-dimethylaminopyridine ( $89 \mathrm{mg}, 0.73 \mathrm{mmol}, 4.9$ equiv) in $\mathrm{CH}_{2} \mathrm{Cl}_{2}(1.0 \mathrm{~mL}+0.5 \mathrm{~mL}$ wash) was added by syringe. The solution was allowed to stir overnight and judged complete by TLC analysis (1:1 hexanes:Et $\left.{ }_{2} \mathrm{O}\right)$. The reaction was diluted with $\mathrm{CH}_{2} \mathrm{Cl}_{2}(3 \mathrm{~mL})$ and washed with saturated $\mathrm{NaHCO}_{3}(2 \mathrm{~mL})$, water $(1 \mathrm{~mL})$, and brine $(2 \mathrm{~mL})$. The organic layer was dried over $\mathrm{MgSO}_{4}$, filtered, and concentrated. The product was purified by gradient silica gel chromato-

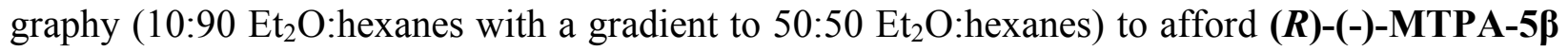
(56 mg, $0.14 \mathrm{mmol}, 92 \%$ yield) as a crystalline white solid $\left(\mathbf{m p} 86-88^{\circ} \mathrm{C}\right) .[\alpha]^{20}{ }_{\mathrm{D}}:-80^{\circ}(c=2.0$, $\left.\mathrm{CHCl}_{3}\right){ }^{1} \mathbf{H}-\mathbf{N M R}\left(400 \mathrm{MHz}, \mathrm{CDCl}_{3}\right): \delta$ 7.53-7.50 (m, 2H), 7.42-7.38 (m, 3H), 6.25 (dd, J=2.5, $1.5 \mathrm{~Hz}, 1 \mathrm{H}), 6.21(\mathrm{dd}, J=2.5,0.5 \mathrm{~Hz}, 1 \mathrm{H}), 5.23(\mathrm{t}, J=2.1 \mathrm{~Hz}, 1 \mathrm{H}), 3.66(\mathrm{~s}, 3 \mathrm{H}), 3.54(\mathrm{q}, J=1.2$ $\mathrm{Hz}, 3 \mathrm{H}), 3.21(\mathrm{tdd}, J=8.3,1.4,0.5 \mathrm{~Hz}, 1 \mathrm{H}), 2.78(\mathrm{dd}, J=8.2,4.8 \mathrm{~Hz}, 1 \mathrm{H}), 2.74(\mathrm{dd}, J=7.7,4.8$ $\mathrm{Hz}, 1 \mathrm{H}), 2.08-2.03(\mathrm{~m}, 2 \mathrm{H}), 1.99-1.89(\mathrm{~m}, 1 \mathrm{H}), 1.75(\mathrm{dt}, J=12.7,3.5 \mathrm{~Hz}, 1 \mathrm{H}) .{ }^{13} \mathbf{C}-\mathbf{N M R}(100$ $\left.\mathrm{MHz}, \mathrm{CDCl}_{3}\right): \delta 173.5,166.0,139.4,137.0,132.4,129.8,128.6,127.4,124.9,122.0,79.4,60.1$, 55.5, 51.8, 44.0, 41.9, 41.3, 31.9, 29.1. IR (NaCl, thin film): 2948 (w), 1736 (s), 1440 (w), 1269 (m), 1243 (m), 1169 (s), 1122 (m), 1018 (m), 867 (w), 713 (m) cm . ESI-HRMS (m/z): Calc'd for $\mathrm{C}_{21} \mathrm{H}_{21} \mathrm{~F}_{3} \mathrm{NaO}_{5}(\mathrm{M}+\mathrm{Na})$ : 433.1239; Found: 433.1241 
Mosher analysis $^{5}$ for determination of absolute stereochemistry ${ }^{6}$ for compounds (S)-(-)-MTPA$5 \beta \&(R)-(-)-M T P A-5 \beta:$
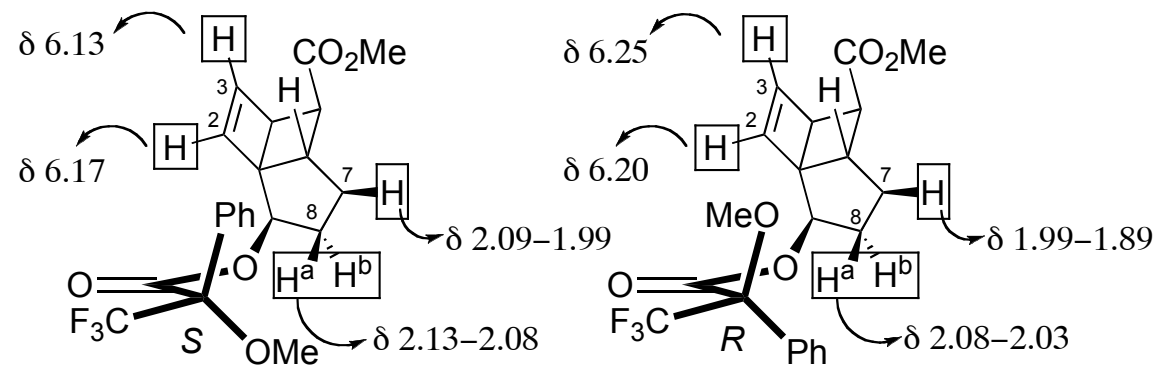

Compound (S)-(-)-MTPA-5 $\beta$

Compound (R)-(-)-MTPA-5 $\beta$

$\begin{array}{cr}\text { Protons } & \Delta \delta_{\mathrm{H}}(\delta(S)-\delta \\ \mathrm{H}(2) & -0.03 \\ \mathrm{H}(3) & -0.12 \\ \mathrm{H}(7) & +0.10 \\ \mathrm{H}^{\mathrm{a}}(8)+\mathrm{H}^{\mathrm{b}}(8) & +0.05\end{array}$

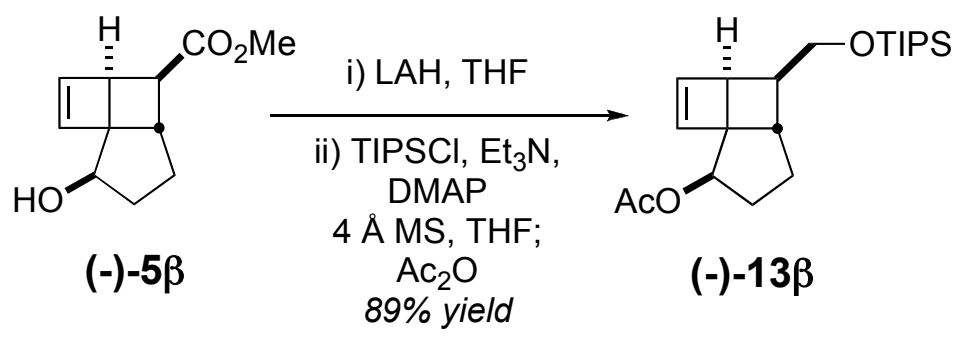

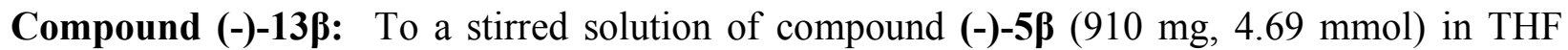
$(42.7 \mathrm{~mL}, 0.11 \mathrm{M})$ cooled to $0{ }^{\circ} \mathrm{C}$, was added a solution of $\mathrm{LAH}(4.2 \mathrm{~mL}, 1 \mathrm{M}$ in $\mathrm{Et} 2 \mathrm{O}, 4.2$ mmol, 0.9 equiv, final concentration $0.1 \mathrm{M}$ ). Once the addition was complete, the solution was allowed to warm to room temperature. The reaction was judged complete after $3 \mathrm{~h}$ by TLC analysis $\left(2: 1 \mathrm{Et}_{2} \mathrm{O}\right.$ :hexanes) and was again cooled to $0{ }^{\circ} \mathrm{C}$. The reaction was quenched by the addition of water $(160 \mu \mathrm{L}), 15 \% \mathrm{NaOH}_{(\mathrm{aq})}(160 \mu \mathrm{L})$, and additional water $(480 \mu \mathrm{L})$ [Note: care must be taken when quenching this reaction due to out gassing]. The reaction was filtered through Celite using THF $(100 \mathrm{~mL})$. The reaction was concentrated to a volume of $\sim 25 \mathrm{~mL}$ and was subsequently filtered through a plug of silica gel $(\sim 1 \mathrm{~cm})$ into a $100 \mathrm{~mL}$ round bottom flask.

5 (a) Rieser, M. J.; Yu-hua, H.; Rupprecht, J. K.; Kozlowski, J. F.; Wood, K. V.; McLaughlin, J. L.; Hanson, R. R.; Zhuang, Z.; Hoye, T. R. J. Am. Chem. Soc. 1992, 114, 10203-10213. (b) Ohtani, I.; Kusumi, T.; Hashman, Y.; Kakisawa, H. J. Am. Chem. Soc. 1991, 113, 4092-4096.

6 It was determined that the stereochemistry obtained from the reduction of compound iii to compound (-)-9 provided the $(R)$ anitpode. 
The filtrate was concentrated to yield a white solid [Note: care must be taken when concentrating as the solution bumps vigorously] which was dried under vacuum $(1 \mathrm{~mm} \mathrm{Hg})$. The solid was used directly in the following reaction.

To the diol was added THF $(9.4 \mathrm{~mL}, 0.5 \mathrm{M})$ and $4 \AA \mathrm{MS}(180 \mathrm{mg}, 20 \mathrm{wt} \%)$, and the solution was allowed to stir at room temperature $(30 \mathrm{~min})$. After the allotted time, triethylamine (2.29 mL, $16.4 \mathrm{mmol}, 3.5$ equiv), 4-dimethylaminopyridine (573 mg, $4.69 \mathrm{mmol}, 1.0$ equiv), and triisopropylsilyl chloride $(1.21 \mathrm{~mL}, 5.63 \mathrm{mmol}, 1.2$ equiv) were added to the reactor. The solution was allowed to stir at room temperature $(24 \mathrm{~h})$ at which point the reaction was judged complete by TLC analysis (2:1 Et ${ }_{2} \mathrm{O}$ :hexanes). To the reactor was then added acetic anhydride ( $887 \mu \mathrm{L}, 9.38 \mathrm{mmol}, 2.0$ equiv) and stirred until the reaction was judged complete ( $1 \mathrm{~h}$ ) by TLC analysis. The reaction was quenched upon the addition of saturated aqueous $\mathrm{NH}_{4} \mathrm{Cl}(50 \mathrm{~mL})$ and $\mathrm{Et}_{2} \mathrm{O}(25 \mathrm{~mL})$. The organic layer was separated, the aqueous layer was extracted with $\mathrm{Et}_{2} \mathrm{O}(3 \mathrm{x}$ $25 \mathrm{~mL})$. The combined organic extracts were washed with water $(25 \mathrm{~mL})$ and brine $(25 \mathrm{~mL})$, dried over $\mathrm{MgSO}_{4}$, filtered, and concentrated. The reaction mixture was purified by gradient silica gel chromatography (2:98 $\mathrm{Et}_{2} \mathrm{O}$ :hexanes with a gradient to 5:95 $\mathrm{Et}_{2} \mathrm{O}$ :hexanes) to afford compound (-)-13ß as a colorless oil (1.525 g, $4.18 \mathrm{mmol}, 89 \%$ yield) which upon standing forms a crystalline white solid $\left(\mathbf{m p} 44.5-46.5^{\circ} \mathrm{C}\right) .[\alpha]^{20}{ }_{\mathrm{D}}:-71^{\circ}\left(c=2.5, \mathrm{CHCl}_{3}\right){ }^{1} \mathbf{H}-\mathbf{N M R}(500 \mathrm{MHz}$, $\left.\mathrm{CDCl}_{3}\right): \delta 6.28(\mathrm{dd}, J=2.5,1.3 \mathrm{~Hz}, 1 \mathrm{H}), 6.20(\mathrm{~d}, J=2.5 \mathrm{~Hz}, 1 \mathrm{H}), 4.98(\mathrm{~d}, J=3.5 \mathrm{~Hz}, 1 \mathrm{H}), 3.68$ (dd, $J=10.0,8.4 \mathrm{~Hz}, 1 \mathrm{H}), 3.58$ (dd, $J=9.9,6.7 \mathrm{~Hz}, 1 \mathrm{H}), 2.95$ (d, $J=8.1 \mathrm{~Hz}, 1 \mathrm{H}), 2.00-1.91(\mathrm{~m}$, 8H), 1.71-1.63 (m, 1H), 1.08-1.02 (m, 21H). ${ }^{13} \mathbf{C}-\mathbf{N M R}\left(125 \mathrm{MHz}, \mathrm{CDCl}_{3}\right)$ : $\delta 171.4,139.8$, $137.1,74.8,64.9,58.9,41.79,41.78,38.8,29.9,28.0,21.3,18.2,12.3$. IR (NaCl, thin film): 2940 (s), 2865 (s), 1736 (s), 1466 (w), 1372(w), 1240 (s), 1121 (m), 1089 (m), 1020 (w), 882 (w), 757 (w), 682 (w). Anal: Calc'd for $\mathrm{C}_{21} \mathrm{H}_{36} \mathrm{O}_{3} \mathrm{Si}: \mathrm{C}, 69.18$; H, 9.95. Found: C, 68.95; H, 10.05 .

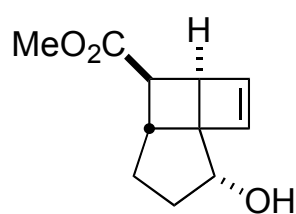

$(+)-5 \alpha$

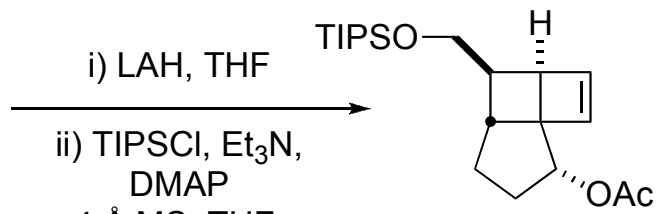

$(+)-13 \alpha$

Compound (+)-13a: To a stirred solution of compound (+)-5a (229 mg, $1.18 \mathrm{mmol})$ in THF $(10.2 \mathrm{~mL}, 0.11 \mathrm{M})$ cooled to $0{ }^{\circ} \mathrm{C}$, was added a solution of $\mathrm{LAH}\left(1.06 \mathrm{~mL}, 1 \mathrm{M}\right.$ in $\mathrm{Et}_{2} \mathrm{O}, 1.06$ 
mmol, 0.9 equiv, final concentration $0.1 \mathrm{M}$ ). Once the addition was complete, the solution was allowed to warm to room temperature. After $3 \mathrm{~h}$, the reaction was judged complete by TLC analysis $\left(2: 1 \mathrm{Et}_{2} \mathrm{O}\right.$ :hexanes). The reaction was again cooled to $0{ }^{\circ} \mathrm{C}$ and quenched by addition of water $(40 \mu \mathrm{L}), 15 \% \mathrm{NaOH}_{(\mathrm{aq})}(40 \mu \mathrm{L})$, and additional water $(120 \mu \mathrm{L})$ [Note: care must be taken when quenching this reaction due to out gassing]. The reaction was filtered through Celite with THF $(50 \mathrm{~mL})$ and the filtrate concentrated. The reaction mixture was then filtered through a plug $(\sim 1 \mathrm{~cm})$ of silica gel with EtOAc into a $25 \mathrm{~mL}$ round bottom flask and again concentrated to yield a colorless oil which was used directly in the following reaction.

To a solution of the diol in THF (2.4 mL, $0.5 \mathrm{M})$ was added $4 \AA \mathrm{MS}$ (46 mg, $20 \mathrm{wt} \%$ ), and allowed to stir at room temperature $(30 \mathrm{~min})$. After the allotted time, triethylamine $(576 \mu \mathrm{L}$, $4.13 \mathrm{mmol}, 3.5$ equiv), 4-dimethylaminopyridine (144 mg, $1.18 \mathrm{mmol}, 1.0$ equiv), and triisopropylsilyl chloride ( $304 \mu \mathrm{L}, 1.42 \mathrm{mmol}, 1.2$ equiv) were added to the reactor. The solution was allowed to stir at room temperature $(24 \mathrm{~h})$ when the reaction was judged complete by TLC analysis $\left(2: 1 \mathrm{Et}_{2} \mathrm{O}\right.$ :hexanes). To the reactor was then added acetic anhydride $(223 \mu \mathrm{L}, 2.36$ mmol, 2.0 equiv) and stirred until the reaction was judged complete $(1 \mathrm{~h})$ by TLC analysis. The reaction was quenched with the addition of a saturated aqueous solution of $\mathrm{NH}_{4} \mathrm{Cl}(25 \mathrm{~mL})$ and $\mathrm{Et}_{2} \mathrm{O}(15 \mathrm{~mL})$. The aqueous layer was extracted with $\mathrm{Et}_{2} \mathrm{O}(3 \times 15 \mathrm{~mL})$. The combined organic extracts were combined, washed with water $(15 \mathrm{~mL})$ and brine $(15 \mathrm{~mL})$, dried over $\mathrm{MgSO}_{4}$, filtered, and concentrated. The reaction mixture was purified by gradient silica gel chromatography $\left(2: 98 \mathrm{Et}_{2} \mathrm{O}\right.$ :hexanes with a gradient to 5:95 $\mathrm{Et}_{2} \mathrm{O}$ :hexanes) to afford compound $(+)-\mathbf{1 3 \alpha}$ (375 mg, $1.03 \mathrm{mmol}, 87 \%$ yield) as a colorless oil. $[\alpha]^{20}{ }_{\mathrm{D}}:+110^{\circ}\left(c=2.5, \mathrm{CHCl}_{3}\right){ }^{1} \mathbf{H}-\mathbf{N M R}$ $\left(400 \mathrm{MHz}, \mathrm{CDCl}_{3}\right): \delta 6.21(\mathrm{dd}, J=2.5,1.2 \mathrm{~Hz}, 1 \mathrm{H}), 6.15(\mathrm{dd}, J=2.5,0.5 \mathrm{~Hz}, 1 \mathrm{H}), 5.14(\mathrm{dd}, J=$ 9.7, $6.3 \mathrm{~Hz}, 1 \mathrm{H}), 3.68(\mathrm{dd}, J=10.0,8.8 \mathrm{~Hz}, 1 \mathrm{H}), 3.56(\mathrm{dd}, J=9.9,7.2 \mathrm{~Hz}, 1 \mathrm{H}), 3.21$ (dd, $J=8.0$, $0.5 \mathrm{~Hz}, 1 \mathrm{H}), 2.17-2.08(\mathrm{~m}, 2 \mathrm{H}), 2.01(\mathrm{~s}, 3 \mathrm{H}), 1.94(\mathrm{dd}, J=7.2,4.8 \mathrm{~Hz}, 1 \mathrm{H}), 1.89-1.78(\mathrm{~m}, 2 \mathrm{H})$, 1.72-1.63 (m, 1H), 1.08-1.00 (m, 21H). ${ }^{13}$ C-NMR (100 MHz, $\left.\mathrm{CDCl}_{3}\right): \delta 170.7,139.4,136.8$, 77.8, 64.9, 60.0, 44.2, 41.1, 39.6, 32.3, 29.7, 21.6, 18.4, 12.9. IR (NaCl, thin film): 3093 (w), 3029 (w), 2937 (s), 2861 (s), 1735 (s), 1465 (m), 1373 (m), 1291 (w), 1245 (s), 1093 (s), 920 $(\mathrm{w}), 883(\mathrm{~m}), 816(\mathrm{w}), 756(\mathrm{~m}), 683(\mathrm{~m}) \mathrm{cm}^{-1}$. Anal: Calc'd for $\mathrm{C}_{21} \mathrm{H}_{36} \mathrm{O}_{3} \mathrm{Si}: \mathrm{C}, 69.18$; H, 9.95. Found: C, 68.99; H, 10.16. 


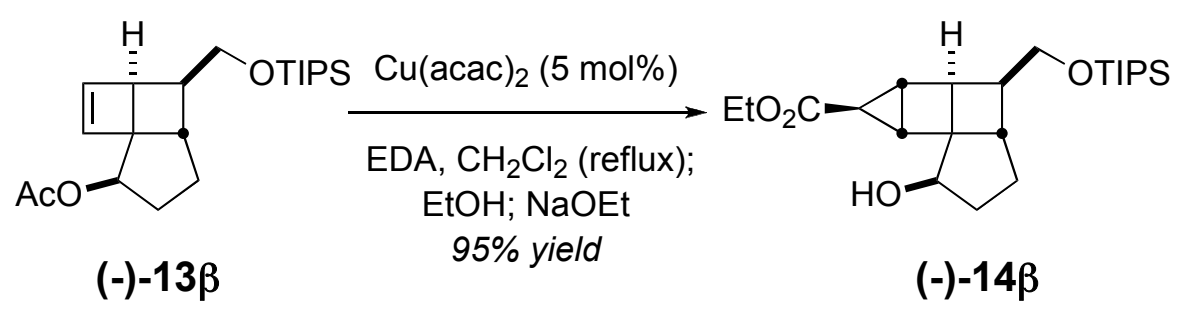

Compound (-)-14ß: [Note equipment for this reaction is as follows: A $100 \mathrm{~mL}$ single neck round bottom flask was fitted with a Claisen adaptor where one port was fitted with a reflux condenser and and the second port was fitted with a Teflon septum where the ethyl diazoacetate was added by syringe] To a refluxing solution of compound (-)-13ß (1.213 g, $3.327 \mathrm{mmol})$ and $\mathrm{Cu}(\mathrm{acac})_{2}$ (44 mg, $0.17 \mathrm{mmol}, 0.05$ equiv) in $\mathrm{CH}_{2} \mathrm{Cl}_{2}(15 \mathrm{~mL}, 0.2 \mathrm{M})$ was added ethyl diazoacetate $(1.58 \mathrm{~mL}, 15.0 \mathrm{mmol}, 5.0$ equiv) with a syringe pump $(0.35 \mathrm{~mL} / \mathrm{h}, 1$ equiv/h). Once addition was complete $(5 \mathrm{~h})$, the solution was allowed stir and reflux for an additional $30 \mathrm{~min}$ at which time the reaction was judged complete by TLC analysis ( $4: 1$ hexanes: $\left.\mathrm{Et}_{2} \mathrm{O}\right)$. The reaction was removed from the heat, allowed to cool (30 $\mathrm{min})$, and then dry ethanol (15 $\mathrm{mL}, 0.1 \mathrm{M})$ was added. The solution was allowed to stir $(15 \mathrm{~min})$, and $\operatorname{NaOEt}_{(s)}(1.02 \mathrm{~g}, 15.0 \mathrm{mmol}, 5.0$ equiv $)$ was added to the reactor and allowed to stir for $1.5 \mathrm{~h}$ after which time the reaction was judged complete by TLC analysis. The reaction was quenched by the addition of saturated aqueous solution of $\mathrm{NH}_{4} \mathrm{Cl}(50 \mathrm{~mL})$, water $(25 \mathrm{~mL})$, and $\mathrm{Et}_{2} \mathrm{O}(25 \mathrm{~mL})$. The organic layer was removed and the aqueous layer was extracted with $\mathrm{Et}_{2} \mathrm{O}(3 \times 25 \mathrm{~mL})$. The organic layers were combined, washed with water $(3 \times 50 \mathrm{~mL})$, brine $(50 \mathrm{~mL})$, dried over $\mathrm{MgSO}_{4}$, filtered, and concentrated to yield a yellow-brown oil. The reaction was purified by gradient silica gel chromatography (5:95 $\mathrm{Et}_{2} \mathrm{O}: \mathrm{CH}_{2} \mathrm{Cl}_{2}$ with a gradient to $\left.10: 90 \mathrm{Et}_{2} \mathrm{O}: \mathrm{CH}_{2} \mathrm{Cl}_{2}\right)$ to yield cyclopropane (-)-14及 $(1.26 \mathrm{~g}, 3.08$ mmol, 93\% yield) as a light yellow oil. $[\alpha]^{20}{ }_{\mathrm{D}}:-55^{\circ}\left(c=2.5, \mathrm{CHCl}_{3}\right) .{ }^{1} \mathbf{H}-\mathbf{N M R}(400 \mathrm{MHz}$, $\left.\mathrm{CDCl}_{3}\right): \delta 4.12(\mathrm{q}, J=7.2 \mathrm{~Hz}, 2 \mathrm{H}), 3.97,(\mathrm{dd}, J=10.0,8.0 \mathrm{~Hz}, 1 \mathrm{H}), 3.87(\mathrm{dd}, J=10.0,6.5 \mathrm{~Hz}$, $1 \mathrm{H}), 3.76(\mathrm{~d}, J=3.3 \mathrm{~Hz}, 1 \mathrm{H}), 2.34(\mathrm{dd}, J=8.1,4.7 \mathrm{~Hz}, 1 \mathrm{H}), 2.22(\mathrm{~s}, 2 \mathrm{H}), 2.18-2.13(\mathrm{~m}, 2 \mathrm{H})$, 2.09-1.99 (m, 1H), 1.85-1.73 (m, 2H), 1.68 (br s, 1H), 1.51 (dd, $J=12.8 .6 .7 \mathrm{~Hz}, 1 \mathrm{H}), 1.26(\mathrm{t}, J$ $=7.1 \mathrm{~Hz}, 3 \mathrm{H}), 1.11-1.00(\mathrm{~m}, 22 \mathrm{H}) .{ }^{13} \mathbf{C}-\mathbf{N M R}\left(100 \mathrm{MHz}, \mathrm{CDCl}_{3}\right): \delta$ 172.7, 71.7, 64.6, 60.5, 56.3, 41.4, 40.4, 39.6, 33.6, 29.7, 27.3, 26.3, 23.1, 18.3, 14.5, 12.3. IR (NaCl, thin film): 3429 (m), 2940 (s), 2865 (s), 1730 (s), 1466 (m), 1385 (m), 1271 (m), 1178 (m), 1096 (m), 882 (m), 682 (w). Anal: Calc'd for $\mathrm{C}_{23} \mathrm{H}_{40} \mathrm{O}_{4} \mathrm{Si}$ : C, 67.60; H, 9.87. Found: C, 67.33; H, 9.59. 


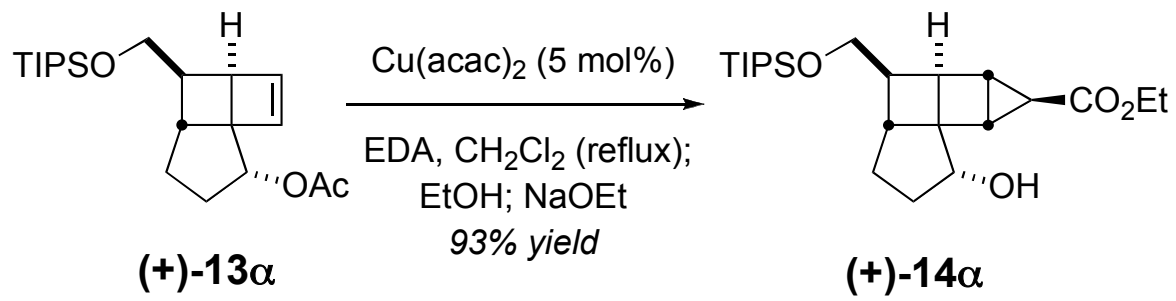

Compound (+)-14a: [Note the equipment for this reaction is as follows: A $25 \mathrm{~mL}$ single neck round bottom flask was fitted with a Claisen adaptor where one port was fitted with a reflux condenser, the second port was fitted with a septum, and ethyl diazoacetate was added with a syringe through the septum] To a refluxing solution of compound $(+)-13 \alpha(316 \mathrm{mg}, 0.867 \mathrm{mmol})$ and $\mathrm{Cu}(\mathrm{acac})_{2}\left(11 \mathrm{mg}, 0.043 \mathrm{mmol}, 0.05\right.$ equiv) in $\mathrm{CH}_{2} \mathrm{Cl}_{2}(4.3 \mathrm{~mL}, 0.20 \mathrm{M})$ was added ethyl diazoacetate $(823 \mu \mathrm{L}, 7.83 \mathrm{mmol}, 9.0$ equiv) with a syringe pump $(0.091 \mathrm{~mL} / \mathrm{h}=1 \mathrm{equiv} / \mathrm{h})$. Once addition was complete $(9 \mathrm{~h})$, the solution was allowed stir and reflux an additional $30 \mathrm{~min}$ at which time the reaction was judged complete by TLC analysis ( $4: 1$ hexanes: $\left.\mathrm{Et}_{2} \mathrm{O}\right)$. The reaction was removed from the heat, allowed to cool $(30 \mathrm{~min})$, and then dry ethanol $(4.3 \mathrm{~mL}$, $0.1 \mathrm{M})$ was added. The solution was allowed to $\operatorname{stir}(15 \mathrm{~min})$ at room temperature, and $\mathrm{NaOEt}_{(s)}$ ( $295 \mathrm{mg}, 3.34 \mathrm{mmol}, 5.0$ equiv) was added to the reactor and allowed to stir for $1.5 \mathrm{~h}$ after which time the reaction was judged complete by TLC analysis ( $4: 1$ hexanes: $\left.\mathrm{Et}_{2} \mathrm{O}\right)$. The reaction was quenched with saturated aqueous $\mathrm{NH}_{4} \mathrm{Cl}(25 \mathrm{~mL})$, water $(25 \mathrm{~mL})$, and $\mathrm{Et}_{2} \mathrm{O}(15 \mathrm{~mL})$. The aqueous layer was extracted with $\mathrm{Et}_{2} \mathrm{O}(3 \mathrm{x} 15 \mathrm{~mL})$. The combined organic extracts were washed with water ( $3 \times 15 \mathrm{~mL})$ and brine $(25 \mathrm{~mL})$, dried over $\mathrm{MgSO}_{4}$, filtered, and concentrated to yield a yellow-brown oil. The reaction was purified by gradient silica gel chromatography (5:95 $\mathrm{Et}_{2} \mathrm{O}: \mathrm{CH}_{2} \mathrm{Cl}_{2}$ with a gradient to $\left.10: 90 \mathrm{Et}_{2} \mathrm{O}: \mathrm{CH}_{2} \mathrm{Cl}_{2}\right)$ to yield cyclopropane (+)-14a (337 mg, 0.824 mmol, 95\% yield) as a colorless oil. $[\alpha]^{20}{ }_{\mathrm{D}}:+38^{\circ}\left(c=2.5, \mathrm{CHCl}_{3}\right) .{ }^{1} \mathbf{H}-\mathbf{N M R}(400$ $\left.\mathrm{MHz}, \mathrm{CDCl}_{3}\right): \delta 4.11(\mathrm{q}, J=4.1 \mathrm{~Hz}, 2 \mathrm{H}), 3.97(\mathrm{dd}, J=9.8,7.1 \mathrm{~Hz}, 1 \mathrm{H}), 3.93-3.90(\mathrm{~m}, 1 \mathrm{H}), 3.87$ $(\mathrm{dd}, J=9.9,7.0 \mathrm{~Hz}, 1 \mathrm{H}), 2.55(\mathrm{dd}, J=7.3,2.7 \mathrm{~Hz}, 1 \mathrm{H}), 2.29-2.18(\mathrm{~m}, 2 \mathrm{H}), 2.15(\mathrm{~d}, J=4.0 \mathrm{~Hz}$, 1H), 2.00-1.94 (m, 1H), 1.77-1.60 (m, 2H), 1.48 (dd, $J=12.6,6.2 \mathrm{~Hz}, 1 \mathrm{H}), 1.27-1.23(\mathrm{~m}, 4 \mathrm{H})$, 1.11-1.03 (m, 21H). ${ }^{13} \mathrm{C}-\mathrm{NMR}\left(100 \mathrm{MHz}, \mathrm{CDCl}_{3}\right): \delta 173.2,75.4,64.8,60.5,54.3,42.5,41.1$, 37.2, 34.4, 29.2, 28.0, 24.4, 22.4, 18.4, 14.6, 12.4. IR ( NaCl, thin film): 3469 (br), 2942 (s), 2859 (s), 1725 (s), 1704 (s), 1463 (m), 1381 (m), 1284 (m), 1117 (s), 1088 (s), 884 (m), 774 (w), $680(\mathrm{~m}) \mathrm{cm}^{-1}$. Anal: Calc'd for $\mathrm{C}_{23} \mathrm{H}_{40} \mathrm{O}_{4} \mathrm{Si}$ : C, 67.60; H, 9.87. Found: C, 67.58; H, 9.86. 


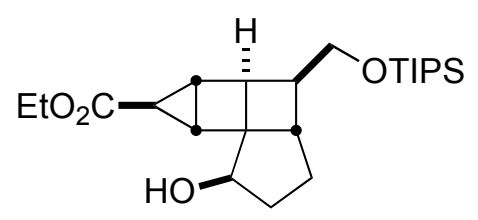

$(-)-14 \beta$

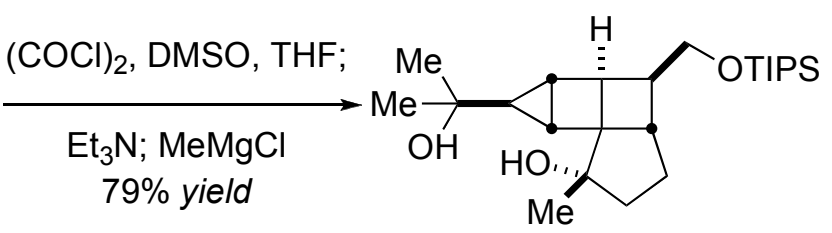

$(-)-4$

Compound (-)-4: To a solution of oxalyl chloride (203 $\mu \mathrm{L}, 2.32 \mathrm{mmol}, 1.80$ equiv) in THF $(6.5 \mathrm{~mL})$ at $-61{ }^{\circ} \mathrm{C}$ was added dimethylsulfoxide $(183 \mu \mathrm{L}, 2.58 \mathrm{mmol}, 2.0$ equiv) dropwise. The solution was allowed to stir (20 min). After the allotted time, a solution of (-)-14ß (526 mg, 1.29 mmol, 1.0 equiv) in THF $(6.5 \mathrm{~mL}$, final concentration $0.1 \mathrm{M})$ was added dropwise to the reactor ( $\sim 10 \mathrm{~min})$. This mixture was again allowed to stir $(30 \mathrm{~min})$. To this mixture was then added (distilled from $\left.\mathrm{CaH}_{2}\right)$ triethylamine $(719 \mu \mathrm{L}, 5.16 \mathrm{mmol}, 4.0$ equiv), this solution was allowed to stir at $-61{ }^{\circ} \mathrm{C}(10 \mathrm{~min})$, and then the cooling bath was removed and allowed to stir at room

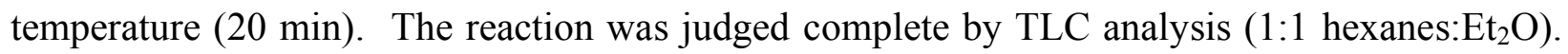
The reactor was then cooled to $-78{ }^{\circ} \mathrm{C}$, diluted with THF $(7.5 \mathrm{~mL}$, final concentration $0.05 \mathrm{M})$, and treated with $\mathrm{MeMgCl}$ (5.4 mL, 2.4 M in THF, $12.9 \mathrm{mmol}, 10.0$ equiv). The solution was allowed to stir at $-78{ }^{\circ} \mathrm{C}(2 \mathrm{~h})$ and then allowed to warm and stir at room temperature $(12 \mathrm{~h})$ at which time the reaction was judged complete by TLC analysis (1:1 Et $\mathrm{E}_{2} \mathrm{O}$ :hexanes). The reaction was cooled in an ice bath and quenched by a slow dropwise addition of saturated aqueous solution of $\mathrm{NH}_{4} \mathrm{Cl}(25 \mathrm{~mL})$ [NOTE: care must be taken when quenching this reaction due to out gassing], water $(10 \mathrm{~mL})$, and EtOAc $(15 \mathrm{~mL})$. The organic layer was removed and the aqueous layer was extracted with EtOAc $(3 \times 25 \mathrm{~mL})$. The combined organic extracts were washed with water $(25 \mathrm{~mL})$ and brine $(25 \mathrm{~mL})$, dried over $\mathrm{MgSO}_{4}$, filtered, and concentrated. The light yellow oil was purified by gradient silica gel chromatography $\left(20: 80\right.$ EtOAc: $\mathrm{CH}_{2} \mathrm{Cl}_{2}$ with a gradient to $100 \%$ EtOAc) to provide compound (-)-4 (418 mg, $1.02 \mathrm{mmol}, 79 \%$ yield) as a clear faint yellow oil. $[\alpha]^{20}{ }_{\mathrm{D}}:-40^{\circ}\left(c=2.5, \mathrm{CHCl}_{3}\right)$. ${ }^{1} \mathbf{H}-\mathbf{N M R}\left(400 \mathrm{MHz}, \mathrm{CDCl}_{3}\right): \delta 3.97$ (dd, $J=9.9$, $7.9 \mathrm{~Hz}, 1 \mathrm{H}), 3.88(\mathrm{dd}, J=9.9,7.4 \mathrm{~Hz}, 1 \mathrm{H}), 2.44(\mathrm{dd}, J=7.3,2.7 \mathrm{~Hz}, 1 \mathrm{H}), 2.27$ (dq, $J=7.4,5.6$ $\mathrm{Hz}, 1 \mathrm{H}), 2.16-2.13(\mathrm{~m}, 1 \mathrm{H}), 1.91-1.82(\mathrm{~m}, 1 \mathrm{H}), 1.76-1.67(\mathrm{~m}, 2 \mathrm{H}), 1.64(\mathrm{dd}, J=4.3,1.0 \mathrm{~Hz}$, $1 \mathrm{H}), 1.50$ (ddd, $J=4.2,2.8,1.3 \mathrm{~Hz}, 1 \mathrm{H}), 1.45-1.39(\mathrm{~m}, 2 \mathrm{H}), 1.25$ (br s, $1 \mathrm{H}), 1.23(\mathrm{~s}, 3 \mathrm{H}), 1.21(\mathrm{~s}$, 3H), 1.14-1.01 (m, 25H). ${ }^{13}$ C-NMR (100 MHz, $\left.\mathrm{CD}_{3} \mathrm{OD}\right): \delta$ 78.6, 70.4, 66.4, 57.1, 44.2, 43.7, 40.9, 38.5, 34.3, 29.7, 29.6, 29.2, 22.5, 21.8, 18.8, 15.5, 13.5. IR ( $\mathrm{NaCl}$, thin film): 3349 (m), 2943 (s), 2858 (s), 1463 (w), 1384 (w), 1099 (m), 874 (w), 777 (w), 686 (w). Anal: Calcd for $\mathrm{C}_{24} \mathrm{H}_{44} \mathrm{O}_{3} \mathrm{Si}: \mathrm{C}, 70.53 ; \mathrm{H}, 10.85$. Found: C, 70.27; H, 10.46 . 


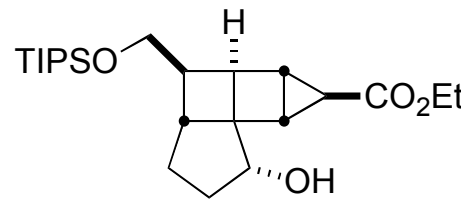

$(+)-14 \alpha$

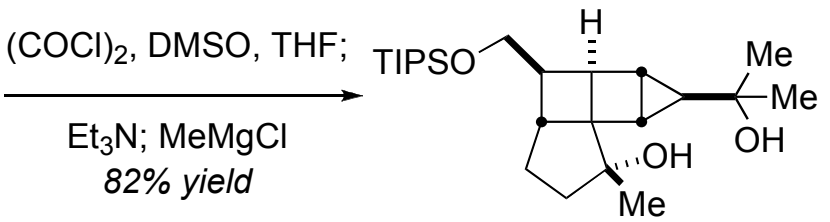

$(+)-4$

Compound (+)-4: To a solution of oxalyl chloride (98 $\mu \mathrm{L}, 1.12 \mathrm{mmol}, 1.80$ equiv) in THF $(3.1 \mathrm{~mL})$ at $-61{ }^{\circ} \mathrm{C}$ (Chloroform/dry ice) under a nitrogen atmosphere was added dimethylsulfoxide ( $89 \mu \mathrm{L}, 1.3 \mathrm{mmol}, 2.0$ equiv) dropwise. The solution was allowed to stir at $-61^{\circ} \mathrm{C}(20 \mathrm{~min})$. After the allotted time, a solution of $\mathbf{1 4 \beta}$ ( $255 \mathrm{mg}, 0.624 \mathrm{mmol}, 1.0$ equiv) in THF ( $3.1 \mathrm{~mL}$ for a final concentration of $0.1 \mathrm{M})$ was added dropwise to the reactor $(\sim 10 \mathrm{~min})$. This mixture was again allowed to stir at $-61{ }^{\circ} \mathrm{C}(30 \mathrm{~min})$. To this mixture was then added (freshly distilled from $\mathrm{CaH}_{2}$ ) triethylamine ( $348 \mu \mathrm{L}, 2.50 \mathrm{mmol}, 4.0$ equiv), this solution was allowed to stir at $-61{ }^{\circ} \mathrm{C}$ (10 $\mathrm{min}$ ), and then the cooling bath was removed and allowed to stir at room temperature (20 min). The reaction was judged complete by TLC analysis ( $1: 1$ hexanes:Et $\left.t_{2} \mathrm{O}\right)$. The reactor was then cooled to $-78^{\circ} \mathrm{C}$, diluted with THF $(3.6 \mathrm{~mL}, 0.05 \mathrm{M})$, and treated with $\mathrm{MeMgCl}(2.6 \mathrm{~mL}$, 2.4 $\mathrm{M}$ in THF, $6.2 \mathrm{mmol}, 10.0$ equiv). The solution was allowed to stir at $-78{ }^{\circ} \mathrm{C}(2 \mathrm{~h})$ and then allowed to warm and stir at room temperature $(12 \mathrm{~h})$ at which time the reaction was judged complete by TLC analysis (1:1 Et $\mathrm{Et}_{2} \mathrm{O}$ hexanes). The reaction was cooled in an ice bath and quenched by the slow dropwise addition of saturated aqueous $\mathrm{NH}_{4} \mathrm{Cl}(15 \mathrm{~mL})$ [NOTE: care must be taken when quenching this reaction due to out gassing], water $(5 \mathrm{~mL})$, and EtOAc $(10 \mathrm{~mL})$. The organic layer was removed and the aqueous layer was extracted with EtOAc ( $3 \times 15 \mathrm{~mL})$. The combined organic extracts were washed with water $(25 \mathrm{~mL})$ and brine $(25 \mathrm{~mL})$, dried over $\mathrm{MgSO}_{4}$, filtered, and concentrated. The light yellow oil was purified by gradient silica gel chromatography (20:80 EtOAc: $\mathrm{CH}_{2} \mathrm{Cl}_{2}$ with a gradient to $100 \% \mathrm{EtOAc}$ ) to yield compound $(+)-4 \quad(210 \mathrm{mg}, 0.513 \mathrm{mmol}, 82 \%$ yield $)$ as a colorless oil which matched both chromatographically and spectroscopically to compound (-)-4 (vide supra). $[\alpha]^{20}{ }_{\mathrm{D}}:+40^{\circ}(c=2.5$, $\left.\mathrm{CHCl}_{3}\right)$. 


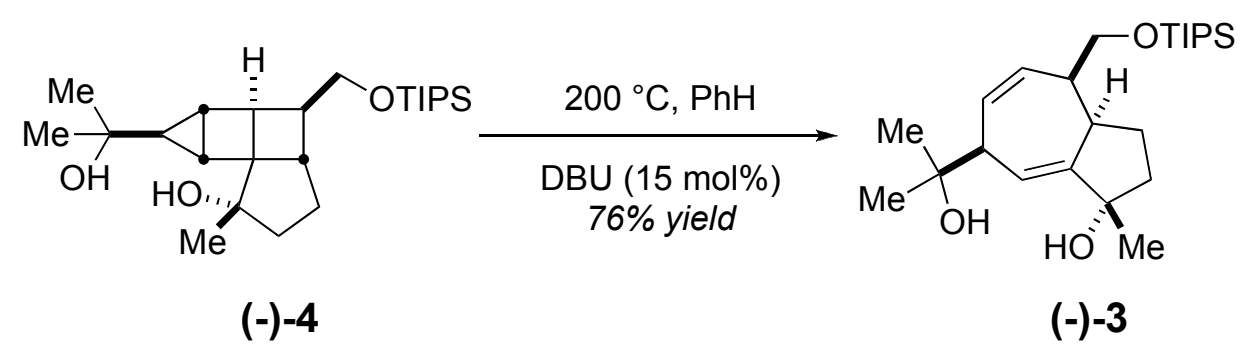

(-)-(1S,3aR,4R,5Z,7S,8E)-7-(2-hydroxypropan-2-yl)-1-methyl-4-((triisopropylsilyloxy)methyl)-1,2,3,3a,4,7-hexahydroazulen-1-ol ((-)-3): A degassed solution of compound (-)-4 (352 mg, $0.861 \mathrm{mmol}), \mathrm{DBU}(19 \mu \mathrm{L}, 0.13 \mathrm{mmol}, 0.15$ equiv), and benzene (43 $\mathrm{mL}, 0.02 \mathrm{M})$ in a $350 \mathrm{~mL}$ glass heavy-wall vessel was placed in a silicon oil bath and heated to $200{ }^{\circ} \mathrm{C}$. The reaction was monitored by NMR and stopped upon complete consumption of starting material $(2.25 \mathrm{~h})$. The reaction was transferred to a separatory funnel with EtOAc $(40 \mathrm{~mL})$ and washed with saturated aqueous solution of $\mathrm{NH}_{4} \mathrm{Cl}(20 \mathrm{~mL})$ and brine $(20 \mathrm{~mL})$. The organic layer was dried over $\mathrm{MgSO}_{4}$, filtered, and concentrated. The resulting yellow oil was purified by basic alumina chromatography (predried at $200{ }^{\circ} \mathrm{C}$ overnight). The substrate was loaded with EtOAc and the column was flushed with EtOAc until a yellow-brown material eluted. At this time, the solvent was switched to 1:99 MeOH:EtOAc with a gradient to 5:95 MeOH:EtOAc to yield compound (-)-3 (266 mg, $0.651 \mathrm{mmol}, 76 \%$ yield) as a white crystalline solid ( $\left.\mathbf{m p} 101-103{ }^{\circ} \mathrm{C}\right) . \quad[\alpha]^{20}$ : $-50^{\circ}\left(c=2.5, \mathrm{CHCl}_{3}\right) .{ }^{1} \mathbf{H}-\mathbf{N M R}\left(400 \mathrm{MHz} \mathrm{CDCl}_{3}\right): \delta 5.89(\mathrm{dt}, J=3.0,1.5 \mathrm{~Hz}, 1 \mathrm{H}), 5.80(\mathrm{ddd}$, $J=12.2,7.1,2.3 \mathrm{~Hz}, 1 \mathrm{H}), 5.64$ (dddd, 12.1, 3.9, 1.5, $0.7 \mathrm{~Hz}, 1 \mathrm{H}), 3.77$ (dd, $J=9.8,7.2 \mathrm{~Hz}, 1 \mathrm{H})$, $3.54(\mathrm{dd}, J=9.8,6.4 \mathrm{~Hz}, 1 \mathrm{H}), 3.31-3.24(\mathrm{~m}, 1 \mathrm{H}), 3.16-3.13(\mathrm{~m}, 1 \mathrm{H}), 2.68-2.61(\mathrm{~m}, 1 \mathrm{H}), 1.92-$ $1.84(\mathrm{~m}, 1 \mathrm{H}), 1.83-1.80(\mathrm{~m}, 1 \mathrm{H}), 1.76-1.67$ (m, 2H), 1.54 (br s, 1H), 1.48 (br s, 1H), $1.38(\mathrm{~s}, 3 \mathrm{H})$, $1.26(\mathrm{~s}, 3 \mathrm{H}), 1.23$ (s, 3H), 1.09-1.01 (m, $21 \mathrm{H}) .{ }^{13} \mathbf{C}-\mathbf{N M R}\left(100 \mathrm{MHz}, \mathrm{CDCl}_{3}\right): \delta$ 152.0, 132.6, $129.1,120.9,80.2,73.6,65.5,51.1,44.4,43.3,41.6,28.2,27.4,27.3,26.5,18.4,12.3$. IR (NaCl, thin film): 3365 (br), 3023 (w), 2940 (s), 2865 (s), 1651 (w), 1464 (m), 1371 (m), 1247 (w), $1175(\mathrm{~m}), 1080$ (s), 883 (m), 775 (m), $679(\mathrm{~m}) \mathrm{cm}^{-1}$. ESI-HRMS (m/z): Calcd for $\mathrm{C}_{24} \mathrm{H}_{44} \mathrm{NaO}_{3} \mathrm{Si}(\mathrm{M}+\mathrm{Na})$ : 431.2957; Found: 431.2952. 


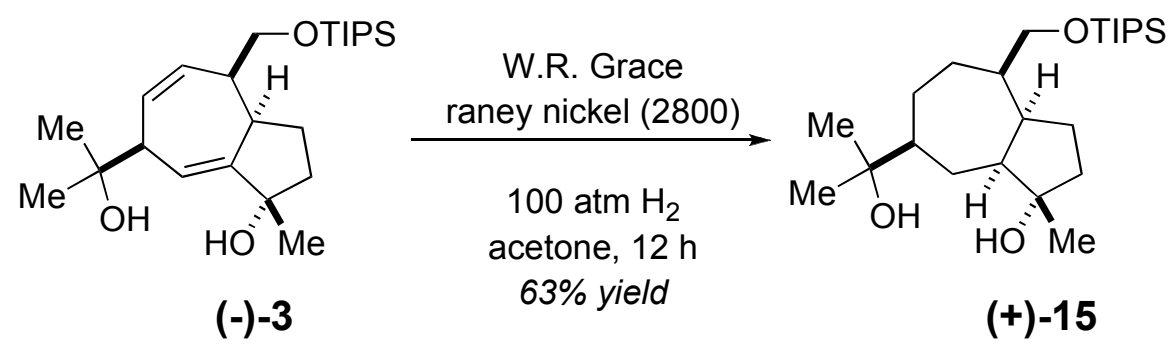

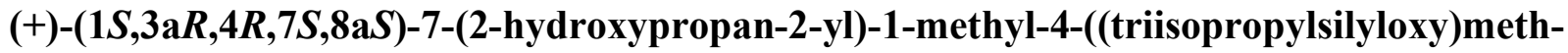

yl)decahydroazulen-1-ol ((+)-15): In a $25 \mathrm{~mL}$ flask was placed $5 \mathrm{~cm}^{3}$ of wet (water) W.R. Grace Raney Nickel 2800 (measured from a $1 \mathrm{~cm}^{3}$ spoon). The catalyst was washed with acetone $(5 \times 10 \mathrm{~mL})$ and the solvent was decanted after each wash. Under a blanket of $\mathrm{N}_{2}$, the acetone was removed until the solvent level reached the top of the catalyst without exposing the catalyst. Immediately, a solution of compound (-)-3 (266 mg, $0.651 \mathrm{mmol})$ in acetone (13 mL, $0.05 \mathrm{M}$ ) was added to the catalyst. The flask was them sealed into a Parr pressure bomb. The atmosphere was purged with $\mathrm{H}_{2}$ (3 x's to $200 \mathrm{psi}$ ) and finally pressurized to the desired pressure (100 atm). The reaction was allowed to stir overnight $(12 \mathrm{~h})$. After the allotted time, the pressure was released from the bomb, the substrate was filtered through Celite atop of silica gel with MeOH:EtOAc (10:90, 50 mL), and concentrated. The resulting clear oil was purified by gradient silica gel chromatography (20:80 EtOAc:hexanes with a gradient to 50:50 EtOAc:hexanes) to yield compound (+)-15 (170 mg, $0.412 \mathrm{mmol}, 63 \%$ yield) as a white solid $\left(\mathbf{m p ~} 116-118^{\circ} \mathrm{C}\right) \cdot[\alpha]^{20}{ }_{\mathrm{D}}:+34^{\circ}\left(c=2.5, \mathrm{CHCl}_{3}\right) .{ }^{1} \mathbf{H}-\mathbf{N M R}\left(400 \mathrm{MHz}, \mathrm{CD}_{3} \mathrm{OD}\right): \delta 3.53(\mathrm{dd}, J=$ 9.5, $6.9 \mathrm{~Hz}, 1 \mathrm{H}), 3.44(\mathrm{dd}, J=9.3,8.3 \mathrm{~Hz}, 1 \mathrm{H}), 2.97-2.91(\mathrm{~m}, 1 \mathrm{H}), 1.99-1.94(\mathrm{~m}, 1 \mathrm{H}), 1.76$ (dd, $J=13.2,2.7 \mathrm{~Hz}, 1 \mathrm{H}), 1.73-1.48(\mathrm{~m}, 7 \mathrm{H}), 1.41-1.34(\mathrm{~m}, 1 \mathrm{H}), 1.31-1.28(\mathrm{~m}, 4 \mathrm{H}), 1.16(\mathrm{~s}, 3 \mathrm{H})$, 1.13-1.07 (m, 24H), $0.92(\mathrm{dt}, J=12.9,8.9 \mathrm{~Hz}, 1 \mathrm{H}) .{ }^{13} \mathbf{C}-\mathbf{N M R}\left(100 \mathrm{MHz}, \mathrm{CDCl}_{3}\right): \delta 82.7,74.3$, $67.9,57.7,47.7,41.4,40.5,38.1,28.3,27.8,26.7,25.6,25.5,22.2,21.1,18.4,12.4$. IR $(\mathrm{NaCl}$, thin film): 3301 (s), 2940 (s), 2870 (s), 1464 (m), 1376 (m), 1254 (w), 1113 (m), 915 (m), 880 (m), 798 (m). Anal: Calcd for $\mathrm{C}_{24} \mathrm{H}_{48} \mathrm{O}_{3} \mathrm{Si}$ : C, 69.84; H, 11.72. Found: C, 69.91; H, 11.67. 


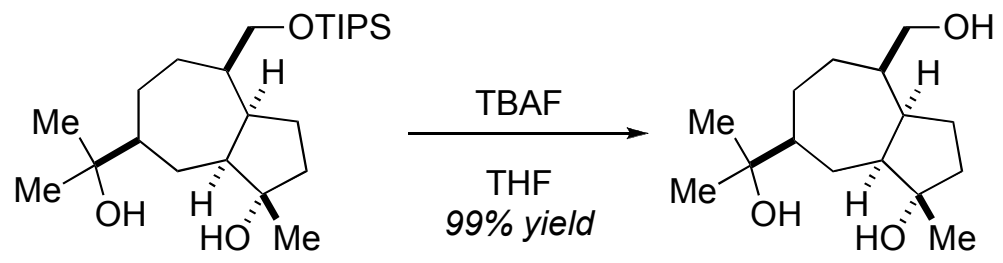

(+)-15

(+)-iv

(+)-(1S,3a $R, 4 R, 7 S, 8 \mathrm{a} S)-4-($ hydroxymethyl)-7-(2-hydroxypropan-2-yl)-1-methyldecahydro-

azulen-1-ol ((+)-iv): To a solution of compound (+)-15 (168 mg, $0.407 \mathrm{mmol})$ in dry THF (2.9 $\mathrm{mL}, 0.1 \mathrm{M})$ was added a solution of tetrabutyammonium fluoride $(1.22 \mathrm{~mL}, 1.0 \mathrm{M}$ in THF, 1.22 mmol, 3.0 equiv). The solution was allowed to stir until the reaction was judged complete (12 h) by TLC analysis. The reaction was quenched by addition of saturated aqueous $\mathrm{NH}_{4} \mathrm{Cl}(25 \mathrm{~mL})$ and EtOAc $(25 \mathrm{~mL})$. The organic layer was removed and the aqueous layer was extracted with EtOAc $(3 \times 20 \mathrm{~mL})$. The combined organic extracts were washed with brine $(20 \mathrm{~mL})$, dried over $\mathrm{MgSO}_{4}$, filtered, and concentrated. The reaction mixture was purified by silica gel chromatography (10:90 MeOH:EtOAc) to yield the desired compound (+)-iv (103 mg, $0.402 \mathrm{mmol}, 99 \%$ yield) as a white solid $\left(\mathbf{m p} 148-149{ }^{\circ} \mathrm{C}\right) .[\alpha]^{20}{ }_{\mathrm{D}}+52^{\circ}(c=1.0, \mathrm{MeOH}) .{ }^{\mathbf{1}} \mathbf{H}-\mathbf{N M R}(500 \mathrm{MHz}$, $\left.\mathrm{CD}_{3} \mathrm{OD}\right): \delta 3.37(\mathrm{dd}, J=10.5,6.9 \mathrm{~Hz}, 1 \mathrm{H}), 3.26(\mathrm{dd}, J=10.5,8.1 \mathrm{~Hz}, 1 \mathrm{H}), 2.93-2.87(\mathrm{~m}, 1 \mathrm{H})$, 1.95-1.89 (m, 1H), $1.76(\mathrm{dd}, J=12.9,2.9 \mathrm{~Hz}, 1 \mathrm{H}), 1.73-1.53(\mathrm{~m}, 8 \mathrm{H}), 1.44(\mathrm{dd}, J=13.7,7.0$, 1H), 1.37 (dddd, $J=12.9,11.3,6.4,2.0 \mathrm{~Hz}, 1 \mathrm{H}), 1.29$ (s, 3H), 1.17 (s, 3H), 1.11 (s, 3H), 0.92 $(\mathrm{dt}, J=13.1,9.0 \mathrm{~Hz}, 1 \mathrm{H}) .{ }^{13} \mathbf{C}-\mathbf{N M R}\left(100 \mathrm{MHz}, \mathrm{CD}_{3} \mathrm{OD}\right): \delta$ 83.3, 74.7, 67.8, 58.4, 48.6, 42.4, 41.4, 38.5, 28.9, 28.1, 27.6, 25.4, 25.1, 22.9, 22.3. IR (KBr Pellet): 3353 (br), 2963 (s), 2923 (s), 2866 (m), 1466 (w), 1384 (m), 1204 (w), 1180 (w), 1162 (m), 1064 (m), 1015 (w), 963 (w), 906 (m) $\mathrm{cm}^{-1}$. Anal: Calcd for $\mathrm{C}_{15} \mathrm{H}_{28} \mathrm{O}_{3}: \mathrm{C}, 70.27 ; \mathrm{H}, 11.01$. Found: C, 70.06; H, 10.86 .

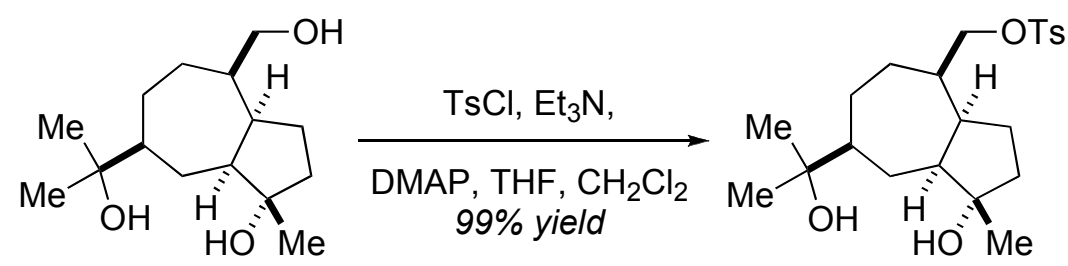

$(+)$-iv

$(+)-\mathbf{V}$

(+)-((1S,3a $R, 4 R, 7 S, 8 \mathrm{a} S)-1-h y d r o x y-7-(2-h y d r o x y p r o p a n-2-y l)-1-m e t h y l d e c a h y d r o a z u l e n-4-$ yl)methyl 4-methylbenzenesulfonate $((+)-\mathbf{v})$ : To a solution of the compound $(+)-i v(59 \mathrm{mg}$, $0.23 \mathrm{mmol})$ in THF $(2.9 \mathrm{~mL})$ and $\mathrm{CH}_{2} \mathrm{Cl}_{2}(2.9 \mathrm{~mL}, 0.04 \mathrm{M})$ was added triethylamine $(460 \mu \mathrm{L}$, $1.15 \mathrm{mmol}, 5.0$ equiv), 4-dimethylaminopyridine (141 $\mathrm{mg}, 1.15 \mathrm{mmol}, 5.0$ equiv), and tosyl 
chloride (219 mg, $1.15 \mathrm{mmol}, 5.0$ equiv). The solution was allowed to stir until the reaction was judged complete $(8 \mathrm{~h})$ by TLC analysis. The reaction was quenched by addition of saturated aqueous $\mathrm{NH}_{4} \mathrm{Cl}(10 \mathrm{~mL})$, and water $(10 \mathrm{~mL})$, and the aqueous layer was extracted with EtOAc (4 x $10 \mathrm{~mL})$. The combined organic extracts were washed with saturated aqueous $\mathrm{NaHCO}_{3}(20$ $\mathrm{mL}$ ) and brine, dried over $\mathrm{MgSO}_{4}$, filtered, and concentrated. The yellow solid obtained upon concentration was purified by gradient silica gel chromatography (loaded with $\mathrm{CH}_{2} \mathrm{Cl}_{2}$, eluted with $\mathrm{Et}_{2} \mathrm{O}$, then EtOAc) to yield compound (+)-v (93 mg, $0.23 \mathrm{mmol}, 99 \%$ yield) as a white solid $\left(\mathbf{m p} 77-79{ }^{\circ} \mathrm{C}\right) .[\alpha]_{\mathrm{D}}^{20}:+40^{\circ}\left(c=2.5, \mathrm{CDCl}_{3}\right){ }^{1} \mathbf{H}-\mathbf{N M R}\left(400 \mathrm{MHz}, \mathrm{CD}_{3} \mathrm{OD}\right): \delta 7.75(\mathrm{~d}, J=8.4$ Hz, 2H), 7.41 (d, $J=8.5$ Hz, 2H), 3.74 (d, $J=7.6$ Hz, 2H), 2.80-2.73 (m, 1H), 2.42 (s, 3H), 2.06$1.99(\mathrm{~m}, 1 \mathrm{H}), 1.69(\mathrm{dd}, J=13.4,3.2 \mathrm{~Hz}, 1 \mathrm{H}), 1.65-1.39(\mathrm{~m}, 8 \mathrm{H}), 1.28-1.18(\mathrm{~m}, 5 \mathrm{H}), 1.09$ (s, $3 \mathrm{H}), 1.03(\mathrm{~s}, 3 \mathrm{H}), 0.79(\mathrm{dt}, J=13.0,9.1,1 \mathrm{H}) .{ }^{13} \mathrm{C}-\mathbf{N M R}\left(100 \mathrm{MHz}, \mathrm{CD}_{3} \mathrm{OD}\right): \delta 146.3,134.5$, 131.0 (br), 129.0 (br), 83.0, 75.7, 74.5, 58.1, 41.9, 38.3, 38.2, 28.4, 28.1, 27.4, 25.2, 25.1, 22.8, 22.1, 21.8. IR (NaCl, thin film): 3306(s), 2960 (s), 2869 (m), 1598 (w), 1463 (w), 1359 (s), 1177 (s), 1097 (m), 954 (s), 835 (m), 811 (m), 664 (m), 557 (m). Anal: Calc'd for $\mathrm{C}_{22} \mathrm{H}_{34} \mathrm{O}_{5} \mathrm{~S}$ : C, 64.36; H, 8.35. Found: C, 64.34; H, 8.63.

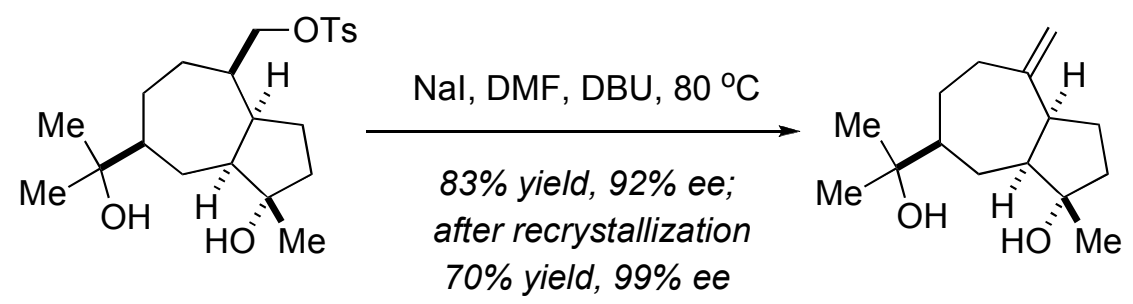

$(+)-\mathbf{v}$

ent-pleocarpenene ((+)-2)

(+)-5-Azulenemethanol, decahydro-3-hydroxy- $\alpha, \alpha, 3$-trimethyl-8-methylene-, $\quad(3 S, 3 \mathrm{a} S, 5 S$, 8aS) (9Cl) (ent-pleocarpenene (+)-2): A solution of compound (+)-v (126 mg, $0.307 \mathrm{mmol})$, sodium iodide (231 mg, $1.54 \mathrm{mmol}, 5.0$ equiv), and 1,8-diazabicyclo[5.4.0]undec-7-ene [DBU] (138 $\mu \mathrm{L}, 0.921 \mathrm{mmol}, 3.0$ equiv) in dimethylformamide $(2.5 \mathrm{~mL}, 0.13 \mathrm{M})$ was heated to $80{ }^{\circ} \mathrm{C}$ in an oil bath until the reaction was judged complete by NMR analysis $(3 \mathrm{~h})$. The reaction was quenched by addition of saturated aqueous $\mathrm{NH}_{4} \mathrm{Cl}(15 \mathrm{~mL})$ and EtOAc $(10 \mathrm{~mL})$. The organic layer was removed and the aqueous layer was extracted with EtOAc $(3 \times 10 \mathrm{~mL})$. The combined organic layers were washed with water $(20 \mathrm{~mL})$ and brine $(20 \mathrm{~mL})$, dried over $\mathrm{MgSO}_{4}$, filtered, and concentrated. The resulting yellow oil was passed through a plug $(\sim 1 \mathrm{~cm})$ of silica gel $\left(\mathrm{CH}_{2} \mathrm{Cl}_{2}\right.$, then $\mathrm{Et}_{2} \mathrm{O}$, and then EtOAc). The sample was collected and concentrated to yield a 
white solid, which was further purified by sublimation $\left(85^{\circ} \mathrm{C}\right.$ at $\left.0.5 \mathrm{~mm} \mathrm{Hg}\right)$ to yield entpleocarpenene (+)-2 (61 mg, $0.26 \mathrm{mmol}, 83 \%$ yield) as a white solid. The white solid can be further purified by recrystallization from hexanes $(61 \mathrm{mg} / 8 \mathrm{~mL}$, overnight $)$ to yield entpleocarpenene (+)-2 (51 mg, $0.21 \mathrm{mmol}, 70 \%$ yield, 99\% ee) as white crystalline needles ( $\mathbf{m p}$ 116-118 ${ }^{\circ} \mathrm{C}$; lit. value mp $\left.133-135{ }^{\circ} \mathrm{C}\right){ }^{7}[\alpha]^{20}{ }_{\mathrm{D}}:+6.1^{\circ}\left(c=1.0, \mathrm{CHCl}_{3}\right)$; lit. value $[\alpha]^{24} \mathrm{D}:-4.77^{\circ}$ $\left(c=0.41, \mathrm{CHCl}_{3}\right){ }^{7}{ }^{1} \mathrm{H}-\mathrm{NMR}\left(500 \mathrm{MHz}, \mathrm{CDCl}_{3}\right): \delta 4.83(\mathrm{br} \mathrm{s}, 1 \mathrm{H}), 4.76(\mathrm{t}, J=1.7 \mathrm{~Hz}, 1 \mathrm{H})$, 3.16-3.10 (m, 1H), 2.51-2.46 (m, 1H), 2.00-1.86 (m, 4H), 1.80 (br d, $J=13.4 \mathrm{~Hz}, 1 \mathrm{H}), 1.76-1.64$ (m, 3H), 1.48 (br s, 1H), 1.39-1.34 (m, 1H), 1.27 (br s, 1H), $1.23(\mathrm{~s}, 3 \mathrm{H}), 1.19-1.13$ (m, $7 \mathrm{H}$ and $1.18(\mathrm{~s}, 3 \mathrm{H})$, and $1.14(\mathrm{~s}, 3 \mathrm{H})), 0.87$ (ddd, $J=13.3,12.1,9.7 \mathrm{~Hz}, 1 \mathrm{H}) .{ }^{13} \mathbf{C}-\mathbf{N M R}(100 \mathrm{MHz}$, $\left.\mathrm{CDCl}_{3}\right): \delta 152.44$ (Quat), $110.25\left(\mathrm{CH}_{2}\right), 82.13$ (Quat), 73.92 (Quat), $54.76(\mathrm{CH}), 52.11(\mathrm{CH})$, $47.53(\mathrm{CH}), 40.28\left(\mathrm{CH}_{2}\right), 36.65\left(\mathrm{CH}_{2}\right), 32.72\left(\mathrm{CH}_{2}\right), 28.25\left(\mathrm{CH}_{3}\right), 28.01\left(\mathrm{CH}_{2}\right), 27.05\left(\mathrm{CH}_{2}\right)$, $26.22\left(\mathrm{CH}_{3}\right), 24.72\left(\mathrm{CH}_{3}\right)$. IR (NaCl, thin film): 3368 (s), 3079 (w), 2967 (s), 2933 (s), 2858 (m), 1637 (w), 1466 (w), 1449 (w), 1374 (m), 1198 (w), 1123 (m), 1069 (w), 935 (w), $885(\mathrm{~m})$, 730 (m). ESI-HRMS (m/z): Calc'd for $\mathrm{C}_{15} \mathrm{H}_{26} \mathrm{NaO}_{2}(\mathrm{M}+\mathrm{Na})$ : 261.1831; Found: 261.1829.

${ }^{7}$ Silva, M.; Wiesenfeld, A.; Sammes, P. G.; Tyler, T. W. Phytochemistry 1977, 16, 379-385. 
(Chiral GC separation conditions: Betadex 120 column (30 m x $0.25 \mathrm{~mm}$ ), 25 psi head pressure, $180{ }^{\circ} \mathrm{C}$ isothermal, FID detector)
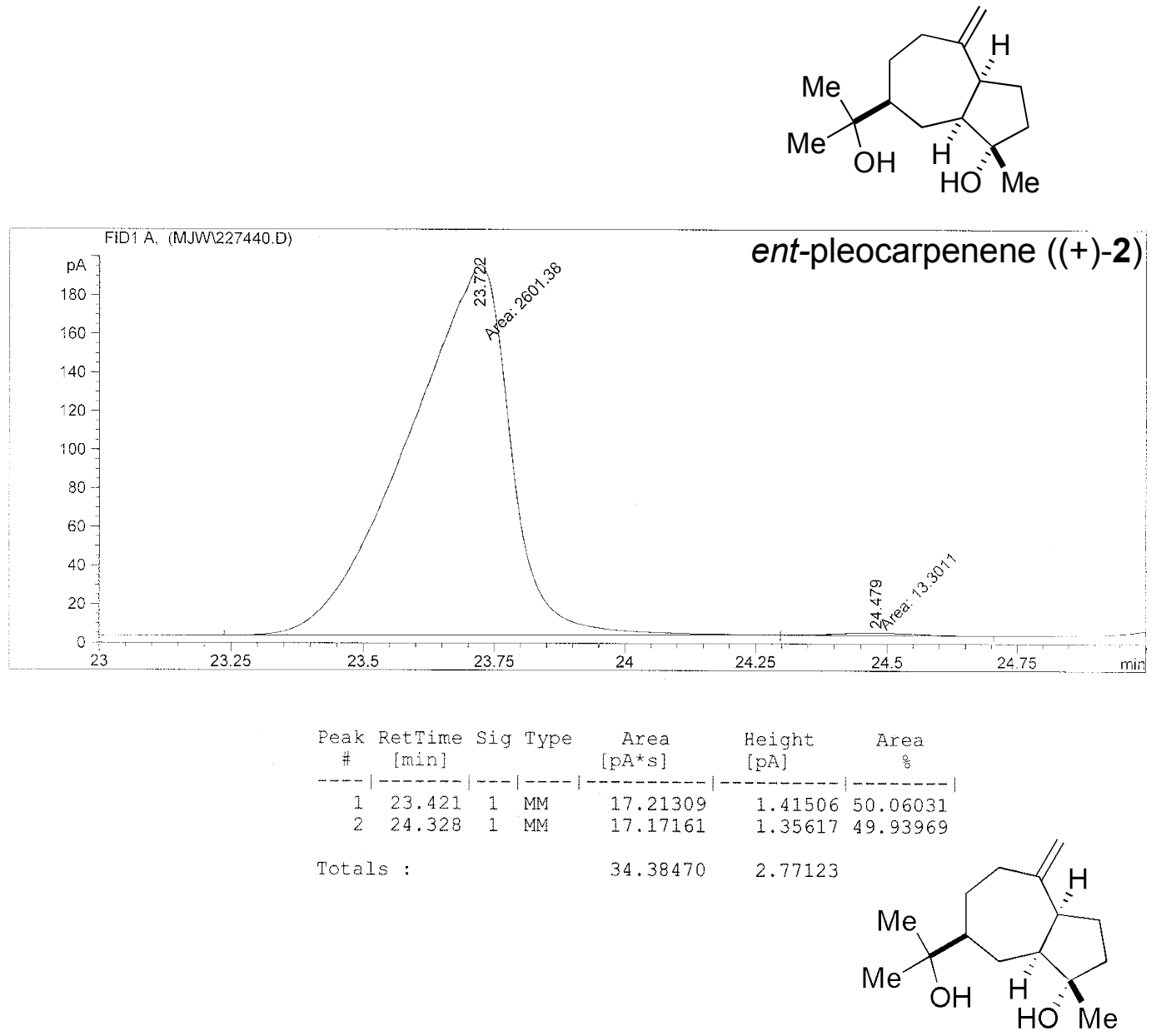

$( \pm$ )-pleocarpenene (2)

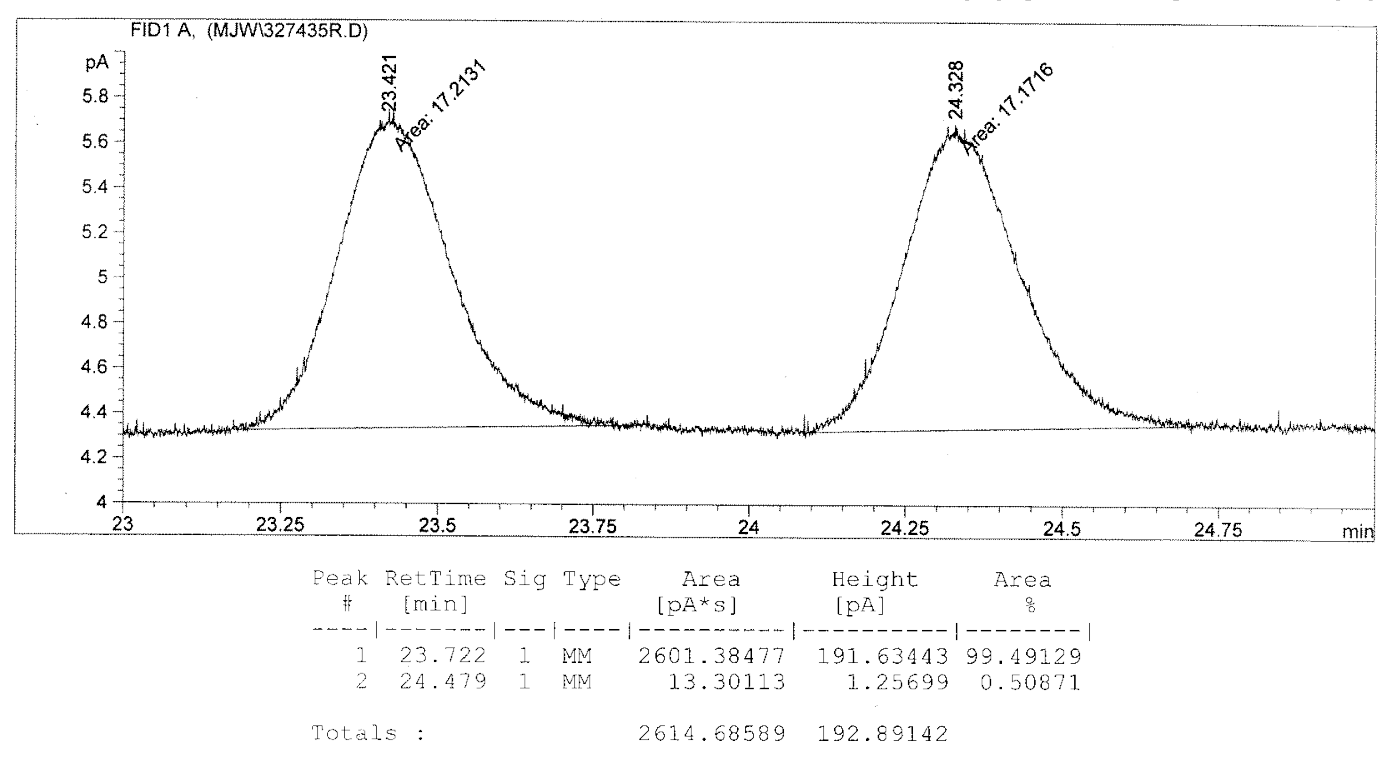




\begin{tabular}{|c|c|c|c|}
\hline \multicolumn{4}{|c|}{${ }^{1} \mathrm{H}$ NMR of Pleocarpenene, $\mathrm{CDCl}_{3}$} \\
\hline \multicolumn{2}{|c|}{ Synthetic (+), $500 \mathrm{MHz}$} & \multicolumn{2}{|c|}{ Literature $^{7, \dagger}(-), 100 \mathrm{MHz}$} \\
\hline Shift (ppm) & Multiplicity/Coupling (Hz) & Shift (ppm) & Multiplicity/Coupling (Hz) \\
\hline 4.83 & broad s & 4.72 & broad s \\
\hline 4.76 & $\mathrm{t}, J=1.7 \mathrm{~Hz}$ & 4.64 & broad $\mathrm{s}$ \\
\hline $3.16-3.10$ & multiplit & & \\
\hline $2.51-2.46$ & multiplit & & \\
\hline $2.00-1.86$ & multiplit & & \\
\hline 1.8 & broad d, $J=13.4$ & & \\
\hline $1.76-1.64$ & multiplit & & \\
\hline 1.48 & broad s & & \\
\hline $1.39-1.34$ & multiplit & & \\
\hline 1.27 & broad s & & \\
\hline 1.23 & $\mathrm{~s}$ & 1.12 & $\mathrm{~s}$ \\
\hline 1.18 & $\mathrm{~s}$ & 1.08 & $\mathrm{~s}$ \\
\hline 1.14 & $\mathrm{~s}$ & 1.04 & $\mathrm{~s}$ \\
\hline $1.19-1.13^{*}$ & multiplit & & \\
\hline 0.87 & $\mathrm{ddd}, J=13.3,12.1,9.7 \mathrm{~Hz}$ & & \\
\hline
\end{tabular}

\begin{tabular}{|c|c|c|}
\hline \multicolumn{3}{|c|}{${ }^{13} \mathrm{C}$ NMR of Pleocarpenene, $\mathrm{CDCl}_{3}$} \\
\hline Synthetic (+), $125 \mathrm{MHz}$ & Literature $^{7}(-), 25 \mathrm{MHz}$ & $\Delta \delta$ \\
\hline Shift (ppm)/Multiplicity ${ }^{\dagger}$ & Shift (ppm)/Multiplicity ${ }^{*}$ & Shift (ppm) \\
\hline 152.44 (Quat) & 152.28 (Quat) & -0.16 \\
\hline $110.25(\mathrm{CH} 2)$ & $109.9(\mathrm{CH} 2)$ & -0.35 \\
\hline 82.13 (Quat) & 81.63 (Quat) & -0.5 \\
\hline 73.92 (Quat) & 73.83 (Quat) & -0.09 \\
\hline $54.76(\mathrm{CH})$ & $54.04(\mathrm{CH})$ & -0.72 \\
\hline $52.11(\mathrm{CH})$ & $52.02(\mathrm{CH} 2)$ & -0.09 \\
\hline $47.53(\mathrm{CH})$ & $47.34(\mathrm{CH})$ & -0.19 \\
\hline $40.28(\mathrm{CH} 2)$ & $40.25(\mathrm{CH} 2)$ & -0.03 \\
\hline $36.65(\mathrm{CH} 2)$ & $37.02(\mathrm{CH} 2)$ & 0.37 \\
\hline $32.72(\mathrm{CH} 2)$ & $32.73(\mathrm{CH} 2)$ & 0.01 \\
\hline $28.25(\mathrm{CH} 3)$ & $28.33(\mathrm{CH} 3)$ & 0.08 \\
\hline $28.01(\mathrm{CH} 2)$ & $27.59(\mathrm{CH})$ & -0.42 \\
\hline $27.05(\mathrm{CH} 2)$ & $26.73(\mathrm{CH} 2)$ & -0.32 \\
\hline $26.22(\mathrm{CH} 3)$ & $25.34(\mathrm{CH} 3)$ & -0.88 \\
\hline $24.72(\mathrm{CH} 3)$ & $24.37(\mathrm{CH} 3)$ & -0.35 \\
\hline
\end{tabular}

$\dagger$ : Carbon multiplicity determined by DEPT \& APT experiments

\#: Carbon multiplicity determined by off resonance pattern 


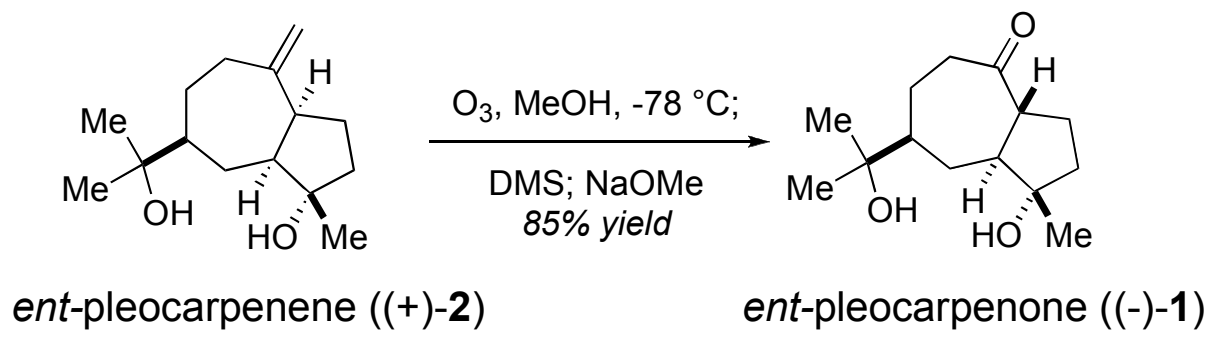

(-)-4(1H)-Azulenone, octahydro-1-hydroxy-7-(1-hydroxy-1-methylethyl)-1-methyl-(1S,3aR, 7S, 8aS)- (9 Cl) (ent-pleocarpenone (-)-1): To a solution of ent-pleocarpenene (+)-(2) (14 mg, $0.06 \mathrm{mmol})$ in dry methanol $(3.0 \mathrm{~mL}, 0.02 \mathrm{M})$ at $-78{ }^{\circ} \mathrm{C}$ was bubbled a stream of ozone $(5 \mathrm{~min})$. The system was then purged with a stream of nitrogen and dimethyl sulfide $(0.2 \mathrm{~mL})$ was added. The $-78{ }^{\circ} \mathrm{C}$ bath was removed and the solution was allowed to stir at room temperature $(2 \mathrm{~h})$. Sodium methoxide (127 $\mu \mathrm{L}$ of a $25 \mathrm{wt} \%$ solution in $\mathrm{MeOH}, 0.59 \mathrm{mmol}, 10.0$ equiv) was added to the solution and allowed to stir at room temperature until the reaction was judged complete (3 h) by TLC analysis (EtOAc). The reaction was quenched with saturated aqueous solution of $\mathrm{NH}_{4} \mathrm{Cl}(5 \mathrm{~mL})$. The aqueous layer was extracted with EtOAc $(4 \times 5 \mathrm{~mL})$. The combined organic extracts were dried over $\mathrm{MgSO}_{4}$, filtered, and concentrated. The light yellow oil was purified by gradient silica gel chromatography (1:1 EtOAc:hexanes with a gradient to $100 \%$ EtOAc) to provide ent-pleocarpenone $(+)-1(12 \mathrm{mg}, 0.05 \mathrm{mmol}, 85 \%$ yield $)$ as a colorless oil. The oil can be recrystallized from 1:1 hexanes:Et $\mathrm{E}_{2} \mathrm{O}$ overnight $\left(\mathbf{m p} 109-111^{\circ} \mathrm{C}\right.$; lit. value: $\mathbf{m p} 96-100{ }^{\circ} \mathrm{C}$ as the hemihydrate and $106-108{ }^{\circ} \mathrm{C}$ as the dry keto-diol). ${ }^{7}[\alpha]^{20}{ }_{\mathrm{D}}:-47^{\circ}\left(c=1.0, \mathrm{CHCl}_{3}\right.$; for the rotation of authentic pleocarpenone see page -S 32). ${ }^{1} \mathbf{H}-\mathbf{N M R}\left(500 \mathrm{MHz}, \mathrm{CDCl}_{3}\right)$ : $\delta 2.86$ (ddd, $J$ $=11.4,10.2,5.0 \mathrm{~Hz}, 1 \mathrm{H}), 2.63(\mathrm{ddd}, J=18.6,5.3,2.8 \mathrm{~Hz}, 1 \mathrm{H}), 2.44(\mathrm{ddd}, J=18.7,12.8,3.3$ $\mathrm{Hz}, 1 \mathrm{H}), 2.37$ (dddd, $J=13.8,9.8,5.0,2.4 \mathrm{~Hz}, 1 \mathrm{H}), 2.23(\mathrm{dq}, J=12.7,2.3 \mathrm{~Hz}, 1 \mathrm{H}), 2.11$ (ddddd, $J=14.6,5.3,3.2,1.9,1.3 \mathrm{~Hz}, 1 \mathrm{H}), 1.72(\mathrm{ddd}, J=12.2,8.4,2.4 \mathrm{~Hz}, 1 \mathrm{H}), 1.68-1.62$ (m, $1 \mathrm{H}), 1.58(\mathrm{td}, J=11.7,2.4 \mathrm{~Hz}, 1 \mathrm{H}), 1.54-1.45$ (m, 2H), 1.39 (br s, $1 \mathrm{H}), 1.30$ (dddd, $J=11.7$, 10.5, 2.9, 1.4 Hz, 1H), 1.25 (s, 3H), $1.21(\mathrm{~s}, 3 \mathrm{H}), 1.17$ (d, J=0.6 Hz, 3H), 1.12 (q, J=11.9 Hz, 1H). ${ }^{13} \mathbf{C}-\mathbf{N M R}\left(125 \mathrm{MHz}, \mathrm{CDCl}_{3}\right): \delta$ 212.87, 81.04, 73.50, 53.39, 53.04, 52.50, 43.11, 40.40, 31.64, 27.90, 26.47, 25.04, 21.73, 20.36 IR (NaCl, thin film): 3395 (br s), 2969 (s), 2938 (m), 2865 (w), 1692 (s), 1467 (w), 1366 (m), 1214 (m), 1187 (m), 1123 (m), 956 (w), 755 (m) cm ${ }^{-1}$. ESI-HRMS (m/z): Calc'd for $\mathrm{C}_{14} \mathrm{H}_{24} \mathrm{NaO}_{3}(\mathrm{M}+\mathrm{Na})$ : 263.1623; Found: 263.1620. 


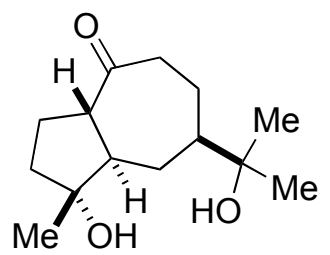

\section{authentic pleocarpenone ((+)-1)}

\section{(+)-4(1H)-Azulenone, octahydro-1-hydroxy-7-(1-hydroxy-1-methylethyl)-1-methyl-(1R,}

3aS, 7R, 8aR)- (9 Cl) (Authentic Pleocarpenone (+)-1) ${ }^{8}$ : white crystalline solid ${ }^{9}$ (mp 108-110 ${ }^{\circ} \mathrm{C}$; lit. value: mp $\left.106-108{ }^{\circ} \mathrm{C}\right)^{7}[\alpha]^{20}{ }_{\mathrm{D}}:+47^{\circ}\left(c=1.0, \mathrm{CHCl}_{3}\right)$; lit. value $[\alpha]_{\mathrm{D}}:-4.77^{\circ}(c=1.0$, $\left.\mathrm{CHCl}_{3}\right)^{7}{ }^{1} \mathbf{H}-\mathbf{N M R}\left(500 \mathrm{MHz}, \mathrm{CDCl}_{3}\right): \delta 2.86(\mathrm{ddd}, J=11.4,10.2,5.1 \mathrm{~Hz}, 1 \mathrm{H}), 2.63(\mathrm{ddd}, J=$ 18.6, 5.4, 2.7 Hz, 1H), 2.44 (ddd, $J=18.7,12.8,3.3 \mathrm{~Hz}, 1 \mathrm{H}), 2.37$ (dddd, $J=13.7,9.8,5.0,2.4$ $\mathrm{Hz}, 1 \mathrm{H}), 2.23(\mathrm{dq}, J=12.7,2.4 \mathrm{~Hz}, 1 \mathrm{H}), 2.12$ (ddddd, $J=14.7,5.4,3.3,2.0,1.2 \mathrm{~Hz}, 1 \mathrm{H}), 1.73$ $(\mathrm{ddd}, J=12.2,8.4,2.4 \mathrm{~Hz}, 1 \mathrm{H}), 1.68-1.61$ (m, 1H), 1.59 (td, $J=11.7,2.4 \mathrm{~Hz}, 1 \mathrm{H}), 1.54-1.46$ (m, 6H, water), 1.31 (dddd, $J=11.6,10.4,2.7,1.1 \mathrm{~Hz}, 1 \mathrm{H}), 1.26$ (s, 3H), 1.21 (s, 3H), 1.18 (d, $J$ $=0.8 \mathrm{~Hz}, 3 \mathrm{H}), 1.12(\mathrm{q}, J=11.8 \mathrm{~Hz}, 1 \mathrm{H}) .{ }^{13} \mathbf{C}-\mathbf{N M R}\left(125 \mathrm{MHz}, \mathrm{CDCl}_{3}\right): \delta 212.81$ (Quat), 81.06 (Quat), 73.52 (Quat), $53.38(\mathrm{CH}), 53.01(\mathrm{CH}), 52.51(\mathrm{CH}), 43.10\left(\mathrm{CH}_{2}\right), 40.42\left(\mathrm{CH}_{2}\right), 31.67$ $\left(\mathrm{CH}_{2}\right), 28.00\left(\mathrm{CH}_{3}\right), 26.43\left(\mathrm{CH}_{3}\right), 25.01\left(\mathrm{CH}_{2}\right), 21.74\left(\mathrm{CH}_{3}\right), 20.36\left(\mathrm{CH}_{2}\right)$. IR $(\mathrm{NaCl}$, thin film): 3401 (br s), 2968 (s), 2935 (s), 2865 (m), 1690 (s), 1460(w), 1369 (m), 1212 (m), 1183 (m), 1122 (s), $957(\mathrm{~m}), 746(\mathrm{w}) \mathrm{cm}^{-1}$. ESI-HRMS (m/z): Calc'd for $\mathrm{C}_{14} \mathrm{H}_{24} \mathrm{NaO}_{3}(\mathrm{M}+\mathrm{Na}): 263.1623$; Found: 263.1633.

ORTEP from X-ray structure: ${ }^{10}$

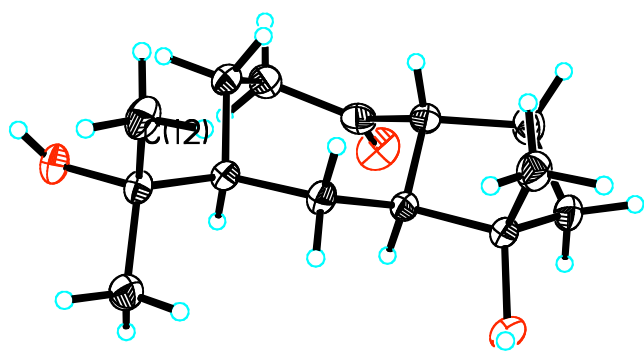

\footnotetext{
8 Authentic pleocarpenone was graciously donated by Prof. Mario Silva of the Universidad de Concepción, Concepción, Chile.

${ }^{9}$ A crystal structure was obtained of the authentic sample. See ORTEP plot (vide supra).

${ }^{10}$ See CIF file for more information.
} 


\begin{tabular}{|c|c|c|c|}
\hline \multicolumn{4}{|c|}{${ }^{\mathbf{1}} \mathbf{H} \mathbf{~ N M R}$ of Pleocarpenone, $\mathbf{C D C l}_{\mathbf{3}}$} \\
\hline & Synthetic (-), 500 MHz & \multicolumn{2}{c|}{ Authentic (+), $\mathbf{5 0 0} \mathbf{~ M H z}$} \\
\hline Shift (ppm) & Multiplicity/Coupling (Hz) & Shift (ppm) & Multiplicity/Coupling (Hz) \\
\hline 2.86 & $\mathrm{ddd}, J=11.4,10.2,5.0 \mathrm{~Hz}$ & 2.86 & $\mathrm{ddd}, J=11.4,10.2,5.1 \mathrm{~Hz}$ \\
\hline 2.63 & $\mathrm{ddd}, J=18.6,5.3,2.8 \mathrm{~Hz}$ & 2.63 & $\mathrm{ddd}, J=18.6,5.4,2.7 \mathrm{~Hz}$ \\
\hline 2.44 & $\mathrm{ddd}, J=18.7,12.8,3.3 \mathrm{~Hz}$ & 2.44 & $\mathrm{ddd}, J=18.7,12.8,3.3 \mathrm{~Hz}$ \\
\hline 2.37 & $\mathrm{dddd}, J=13.8,9.8,5.0,2.4 \mathrm{~Hz}$ & 2.37 & $\mathrm{dddd}, J=13.7,9.8,5.0,2.4 \mathrm{~Hz}$ \\
\hline 2.23 & $\mathrm{dq}, J=12.7,2.3 \mathrm{~Hz}$ & 2.23 & $\mathrm{dq}, J=12.7,2.4 \mathrm{~Hz}$ \\
\hline 2.11 & ddddd, $J=14.6,5.3,3.2,1.9,1.3 \mathrm{~Hz}$ & 2.12 & $\mathrm{ddddd}, J=14.7,5.4,3.3,2.0,1.2 \mathrm{~Hz}$ \\
\hline 1.72 & $\mathrm{ddd}, J=12.2,8.4,2.4 \mathrm{~Hz}$ & 1.73 & $\mathrm{ddd}, J=12.2,8.4,2.4 \mathrm{~Hz}$ \\
\hline $1.68-1.62$ & multiplit & $1.68-1.62$ & multiplit \\
\hline 1.58 & $\mathrm{td}, J=11.7,2.4 \mathrm{~Hz}$ & 1.59 & td, $J=11.7,2.5 \mathrm{~Hz}$ \\
\hline $1.54-1.45$ & multiplit & $1.54-1.46$ & multiplit \\
\hline 1.39 & br s & & $\mathrm{d}$ \\
\hline 1.30 & $\mathrm{dddd}, J=11.7,10.5,2.9,1.4 \mathrm{~Hz}$ & 1.31 & $\mathrm{dddd}, J=11.6,10.4,2.7,1.1 \mathrm{~Hz}$ \\
\hline 1.25 & $\mathrm{~s}$ & 1.26 & $\mathrm{~s}$ \\
\hline 1.21 & $\mathrm{~s}$ & 1.21 & $\mathrm{~d}, J=0.8 \mathrm{~Hz}$ \\
\hline 1.17 & $\mathrm{~d}, J=0.6 \mathrm{~Hz}$ & 1.18 & $\mathrm{q}, J=11.9 \mathrm{~Hz}$ \\
\hline 1.12 & $\mathrm{q}, J=11.9 \mathrm{~Hz}$ & 1.12 & \\
\hline
\end{tabular}

\begin{tabular}{|c|c|c|}
\hline \multicolumn{3}{|c|}{${ }^{\mathbf{1 3}} \mathbf{C}$ NMR of Pleocarpenone, $\mathbf{C D C l}_{\mathbf{3}}$} \\
\hline Synthetic (-), 125 MHz & Authentic (+), 125 MHz & $\boldsymbol{\Delta} \boldsymbol{\delta}$ \\
\hline Shift (ppm) & Shift (ppm)/Multiplicity & Shift (ppm) \\
\hline 212.87 & $212.81($ Quat) & 0.06 \\
\hline 81.04 & $81.06(\mathrm{Quat})$ & -0.02 \\
\hline 73.50 & $73.52(\mathrm{Quat})$ & -0.02 \\
\hline 53.39 & $53.38(\mathrm{CH})$ & 0.01 \\
\hline 53.04 & $53.01(\mathrm{CH})$ & 0.03 \\
\hline 52.50 & $52.51(\mathrm{CH})$ & -0.01 \\
\hline 43.11 & $43.10\left(\mathrm{CH}_{2}\right)$ & 0.01 \\
\hline 40.40 & $40.42\left(\mathrm{CH}_{2}\right)$ & -0.02 \\
\hline 31.64 & $31.67\left(\mathrm{CH}_{2}\right)$ & -0.03 \\
\hline 27.90 & $28.00\left(\mathrm{CH}_{3}\right)$ & -0.10 \\
\hline 26.47 & $26.43\left(\mathrm{CH}_{3}\right)$ & 0.04 \\
\hline 25.04 & $25.01\left(\mathrm{CH}_{2}\right)$ & 0.03 \\
\hline 21.73 & $21.74\left(\mathrm{CH}_{3}\right)$ & -0.01 \\
\hline 20.36 & $20.36\left(\mathrm{CH}_{2}\right)$ & 0.00 \\
\hline
\end{tabular}


$-S 33-$

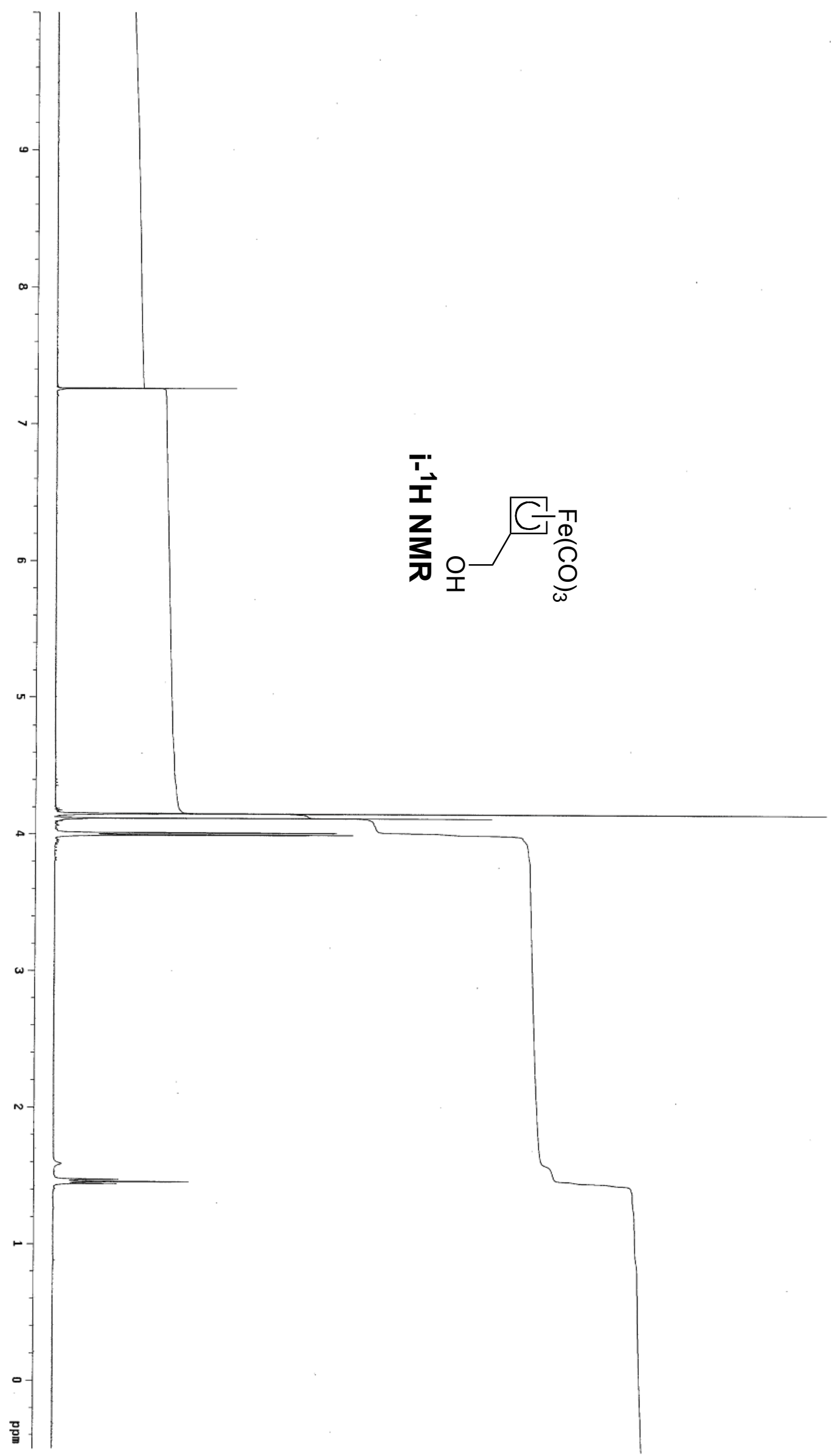


$-S 34$ -

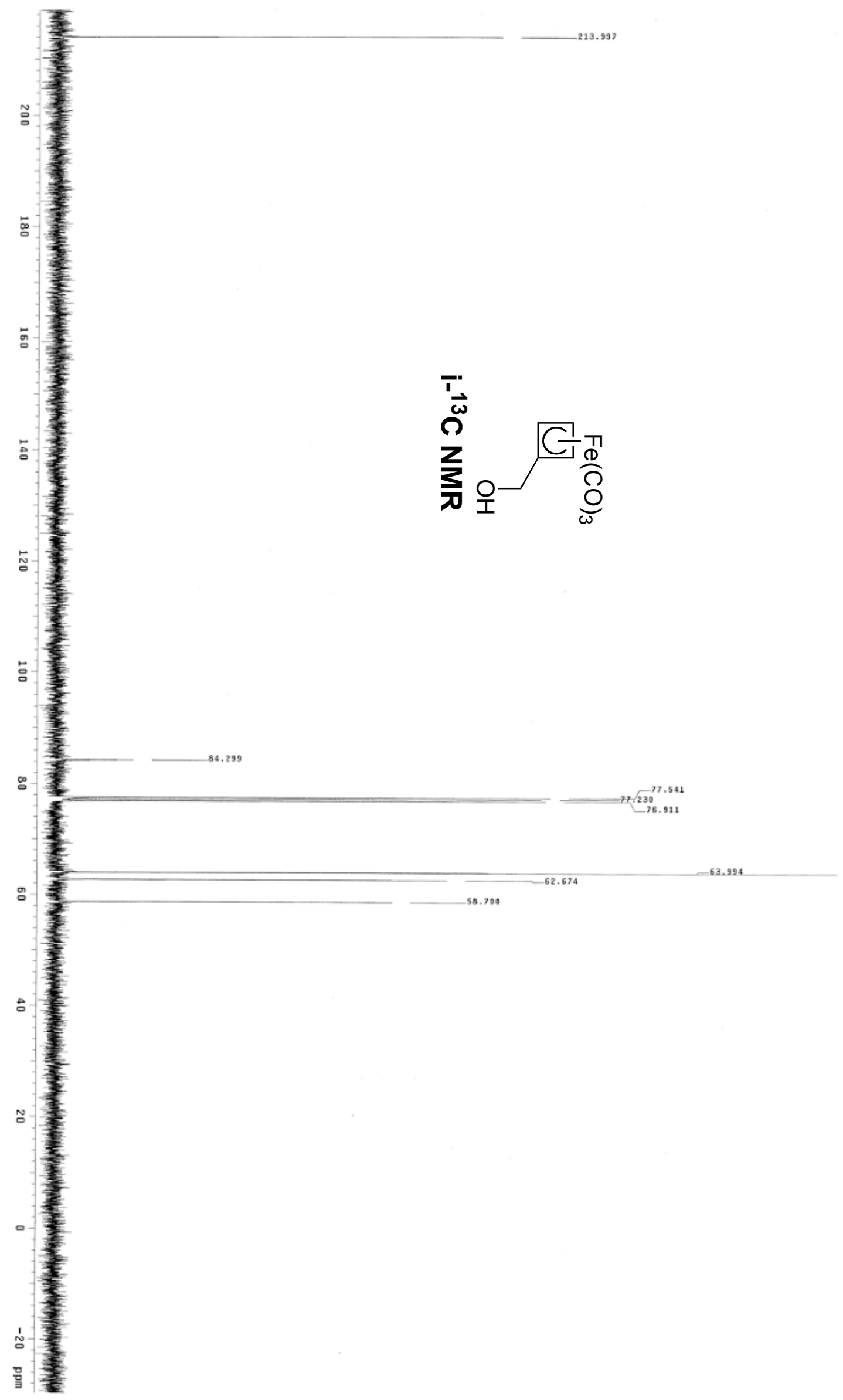


$-S 35-$

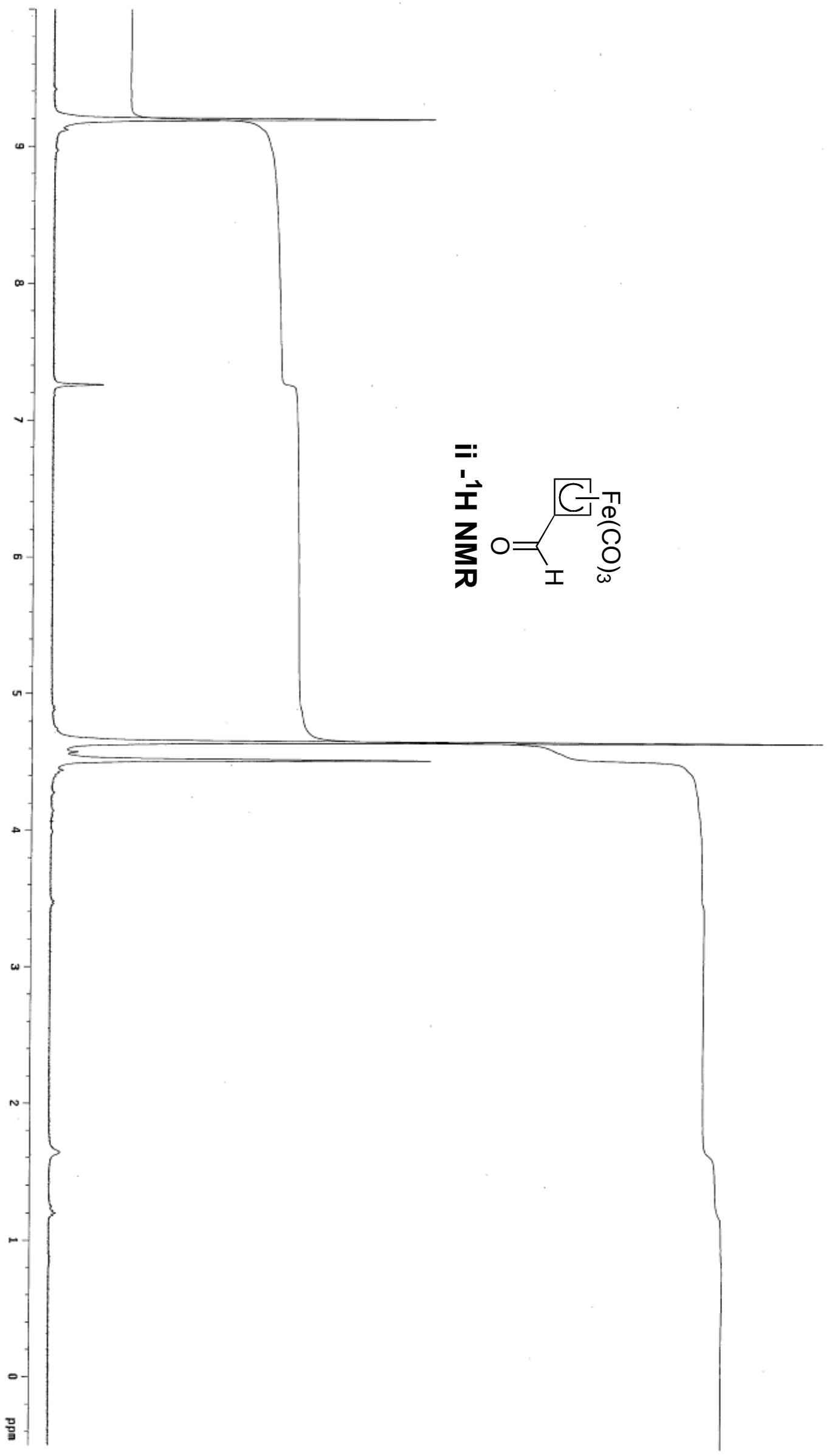


$-S 36-$

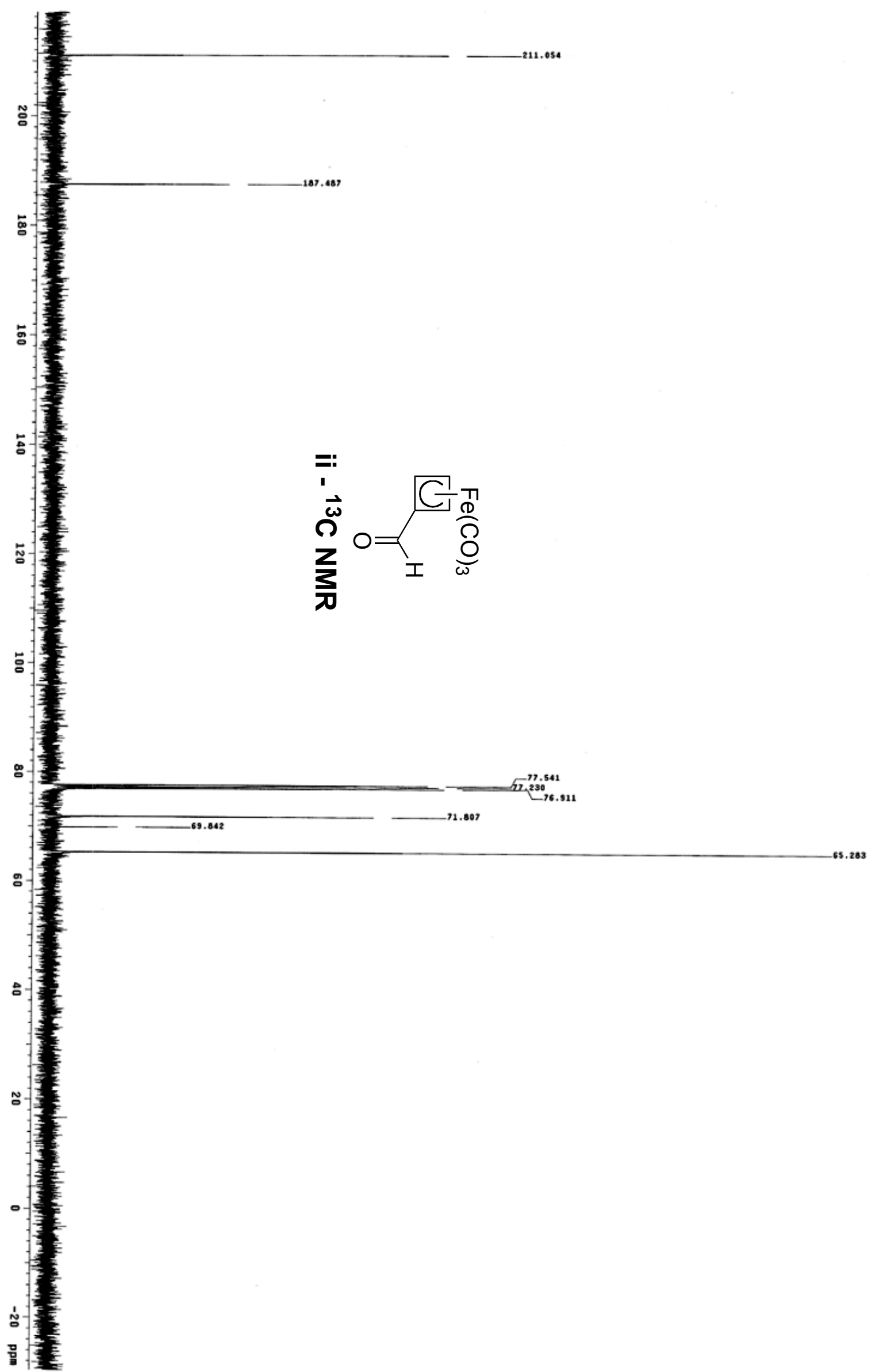


-S 37 -

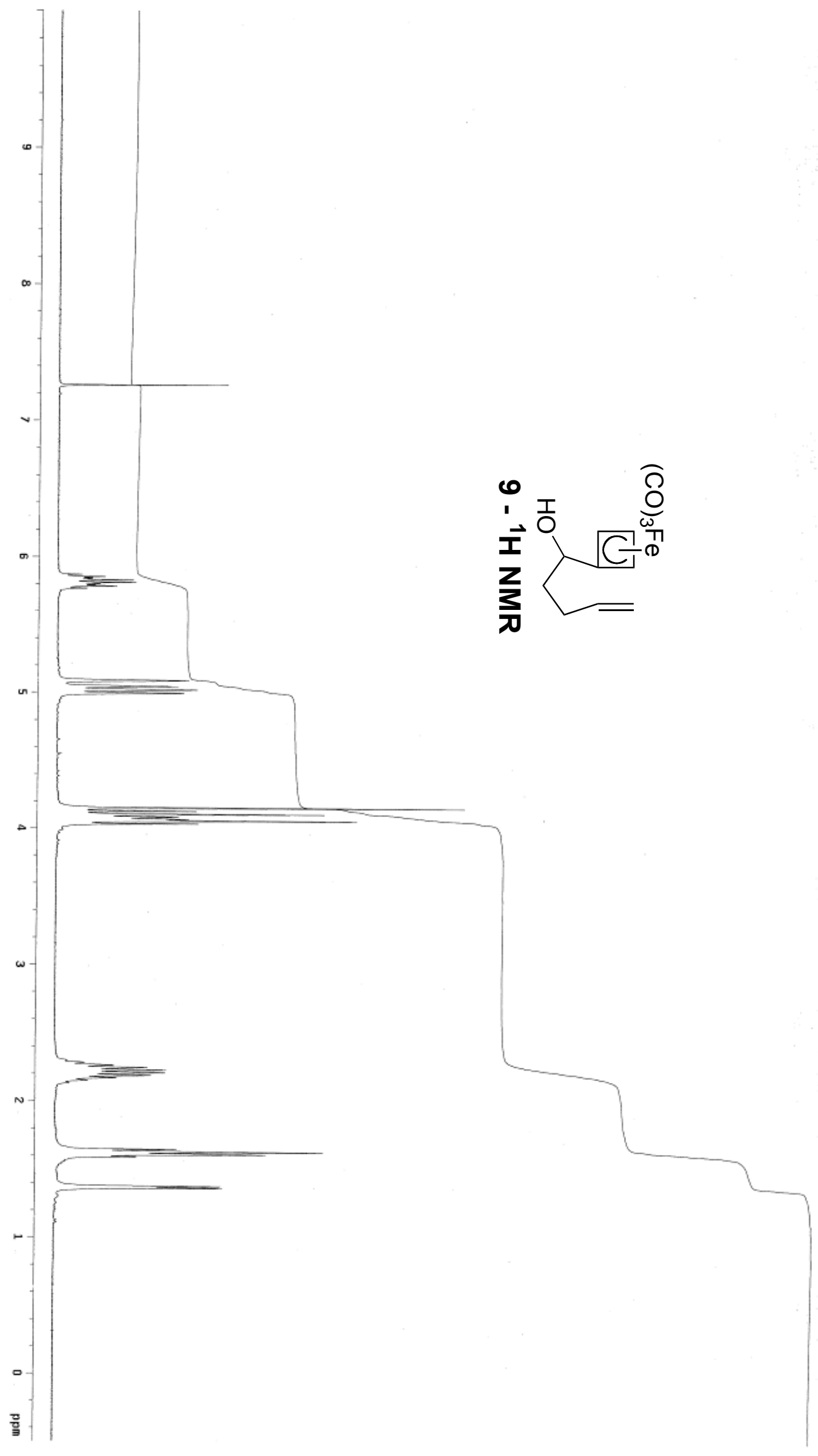



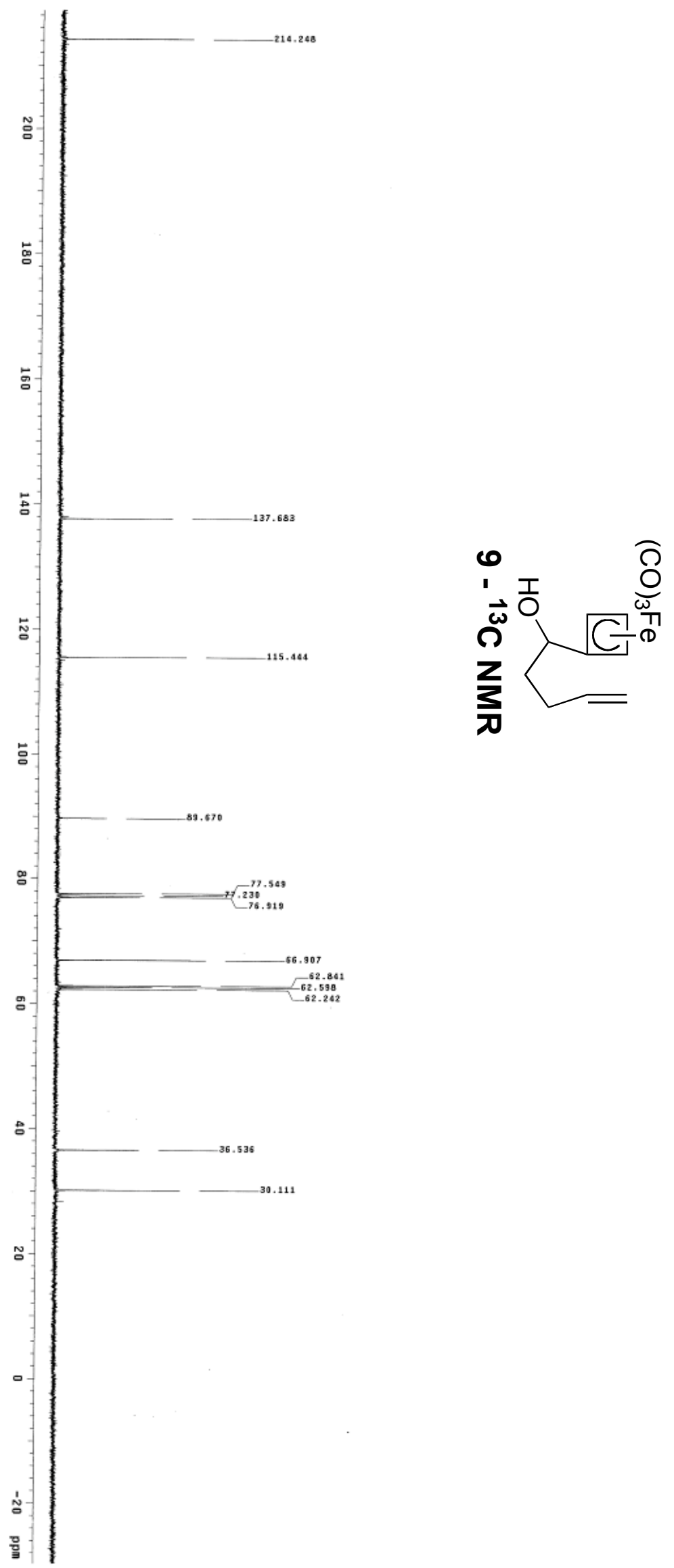
-S 39 -

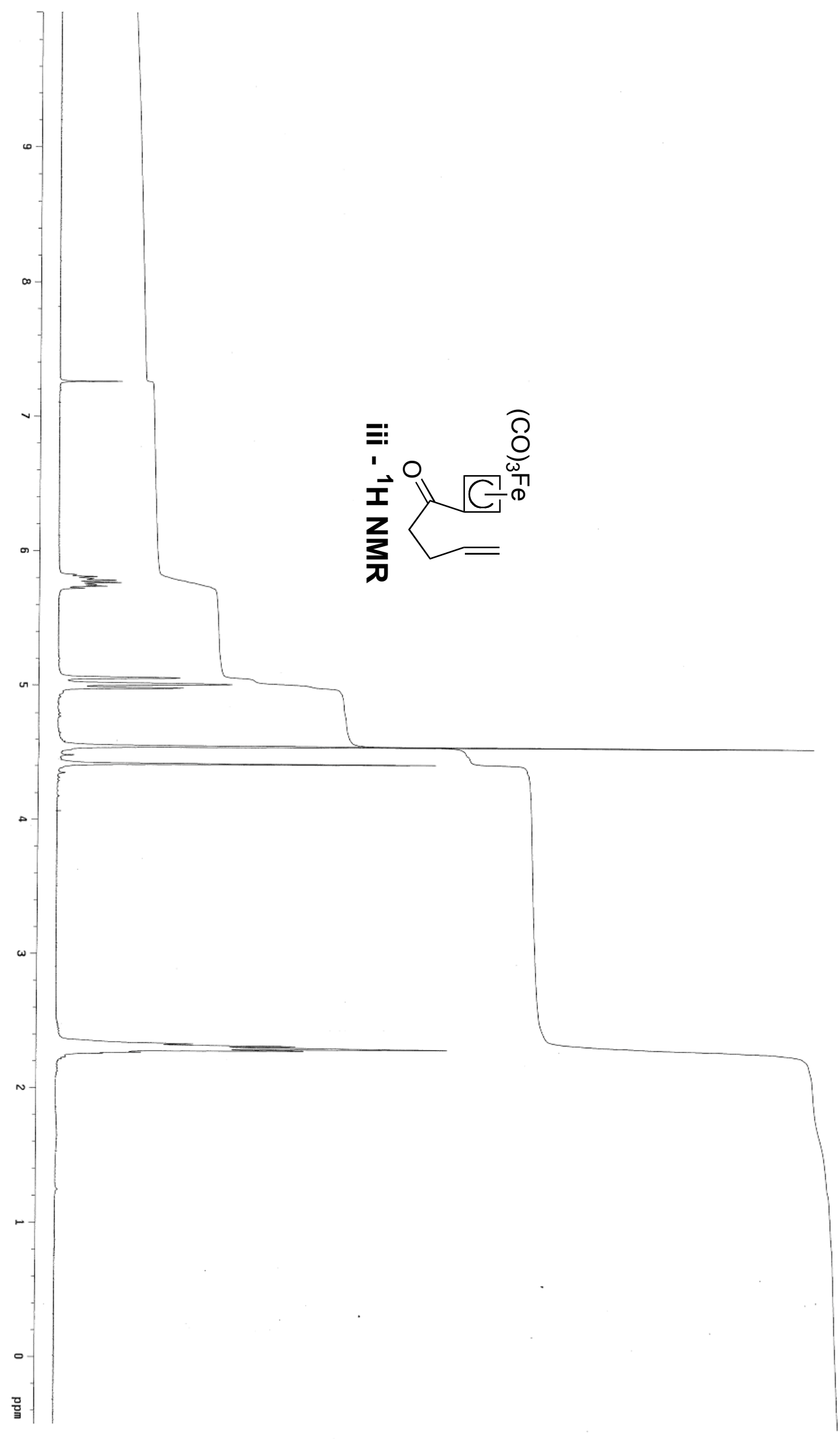




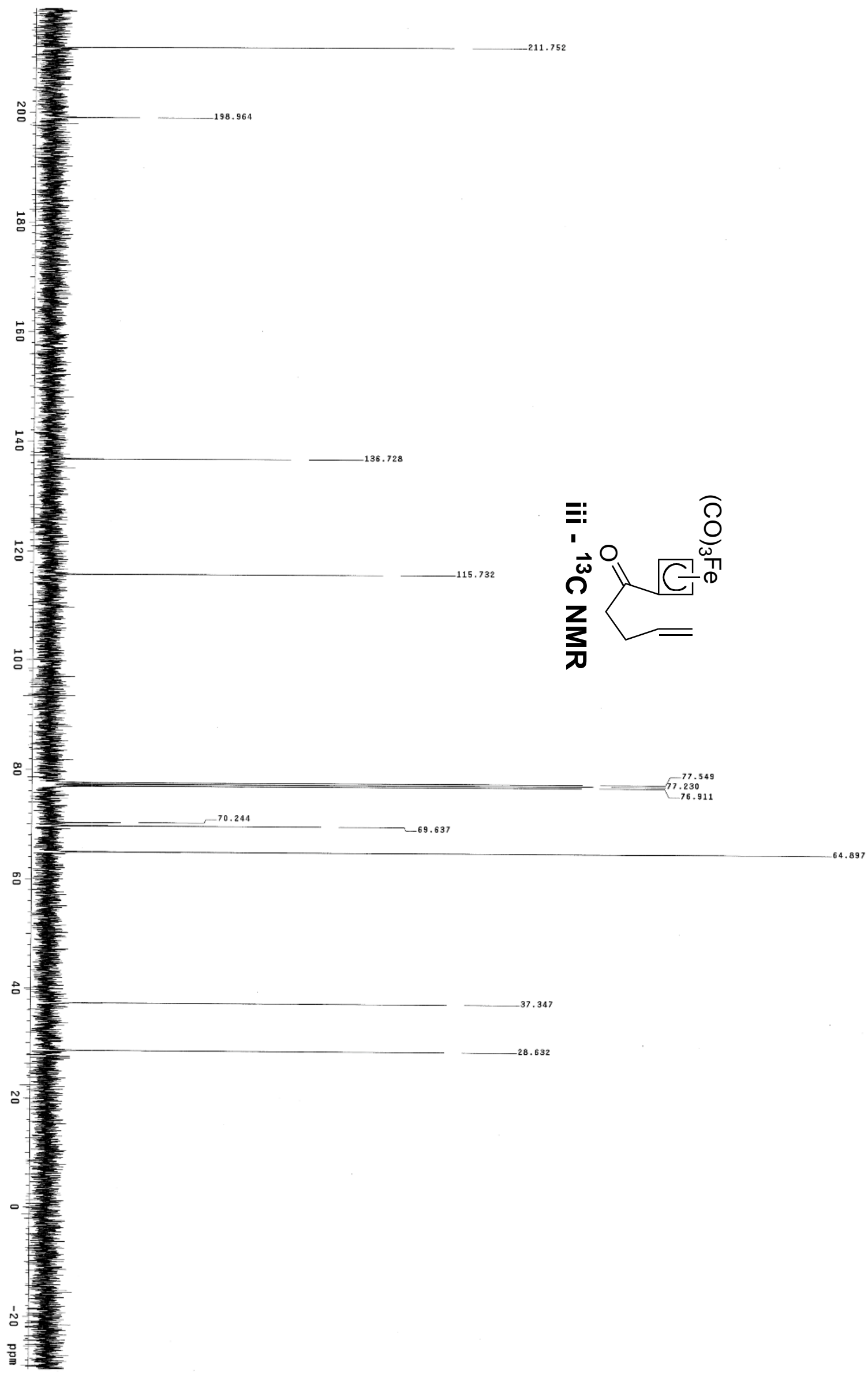


$-S 41-$

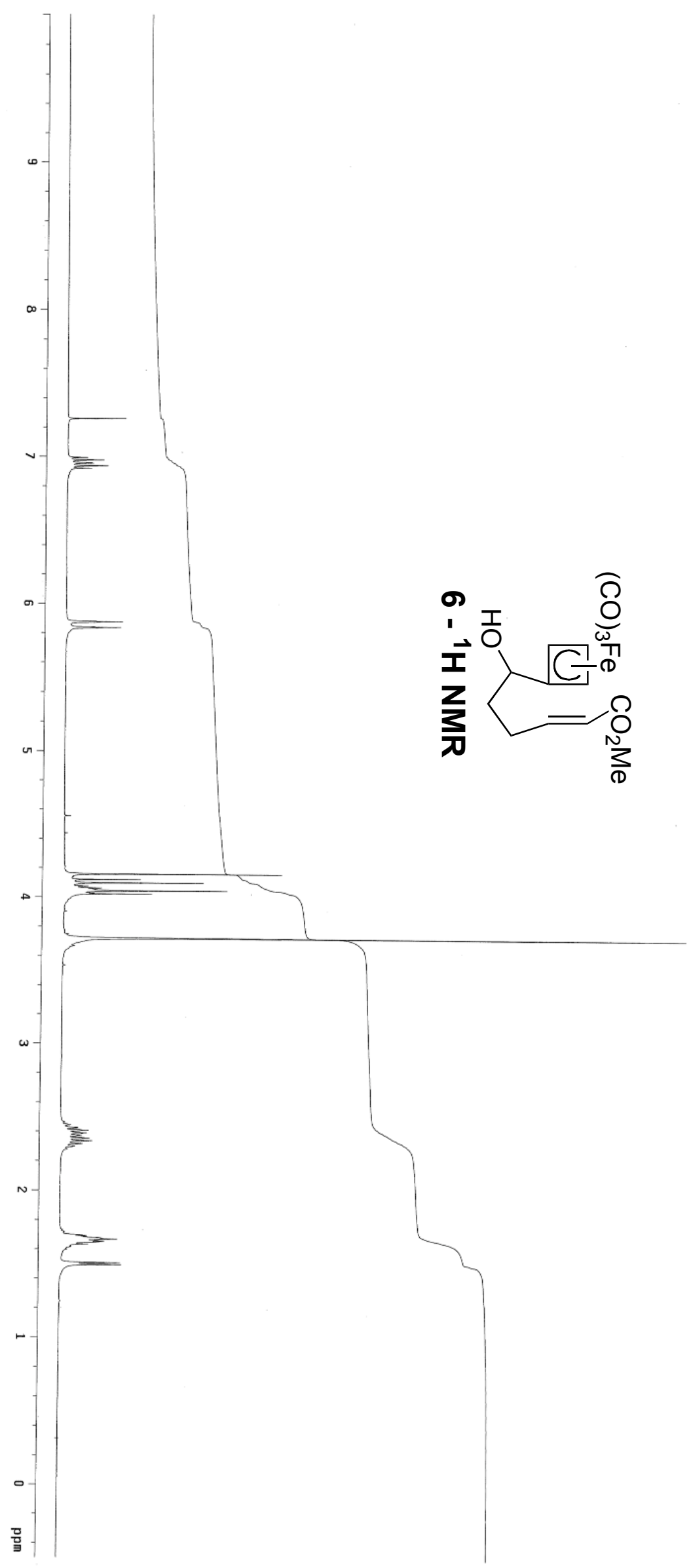




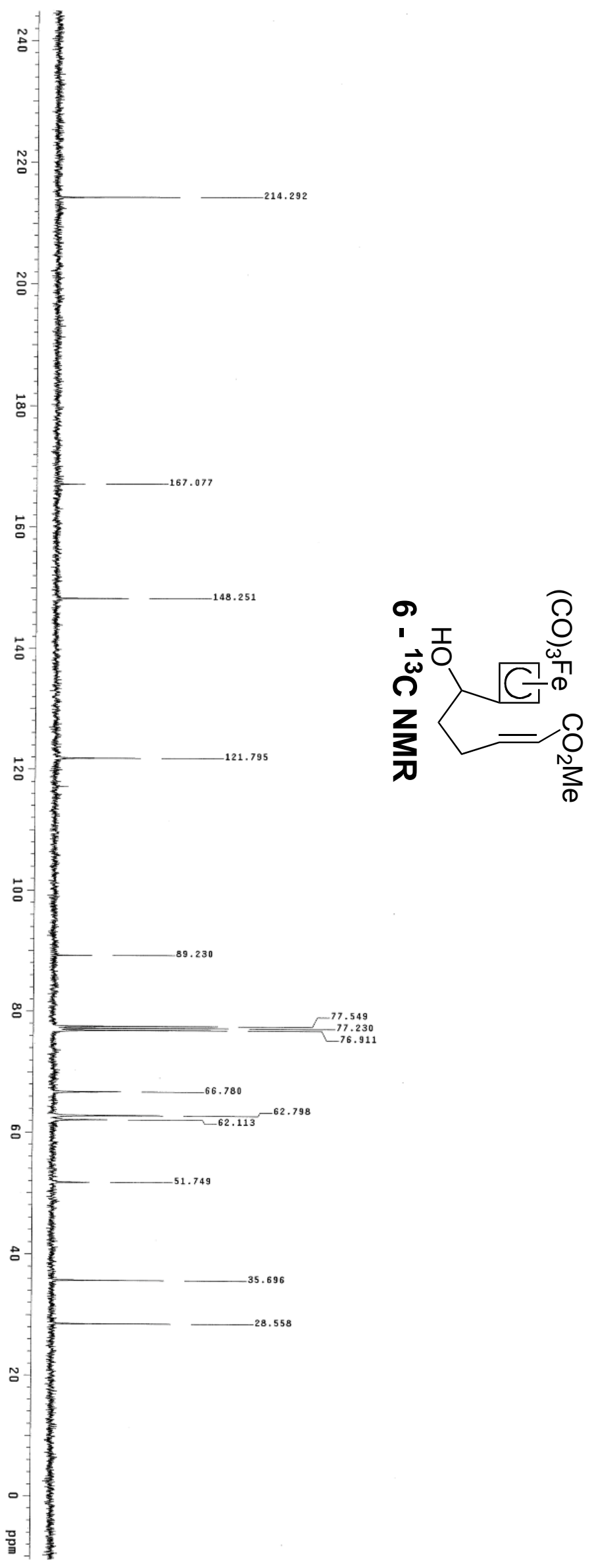


$-S 43$ -

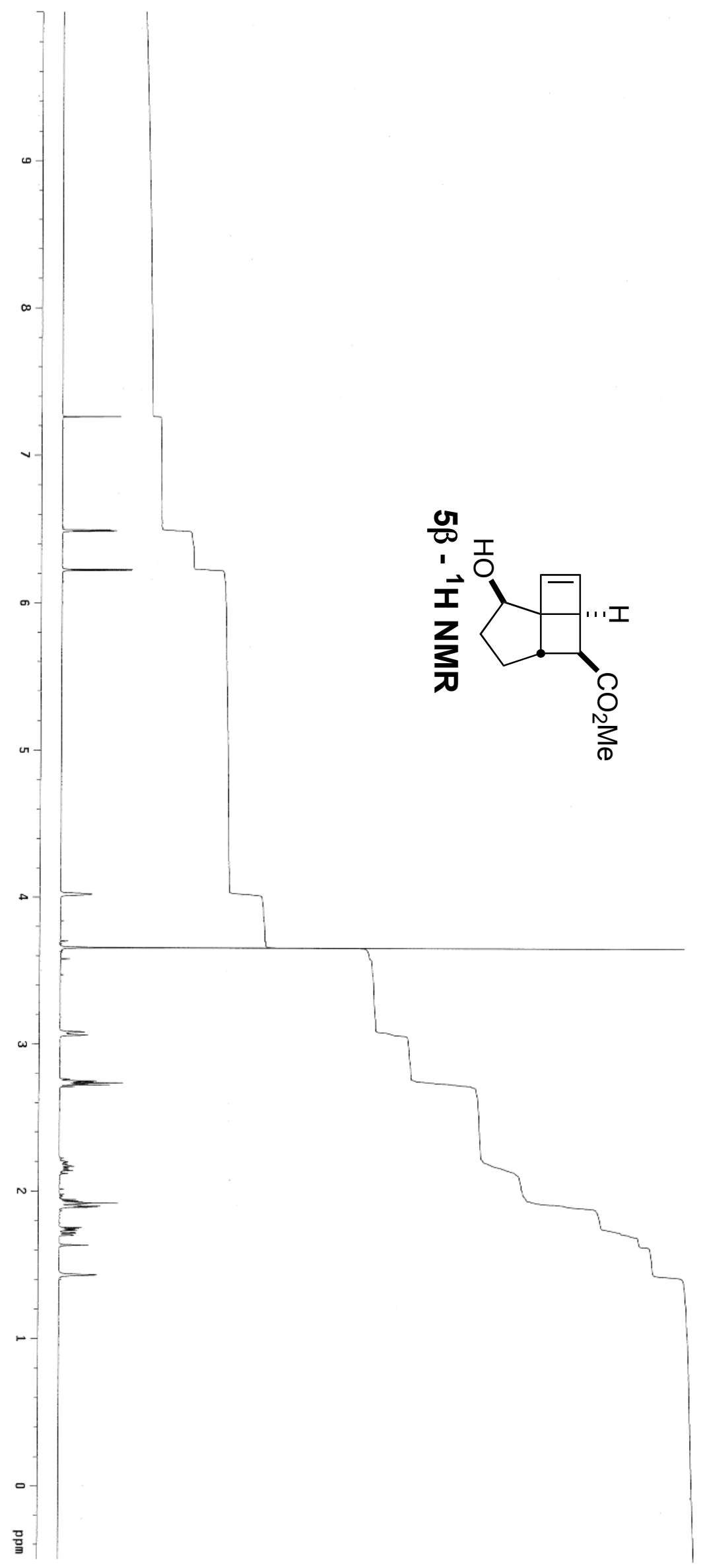


$-S 44$ -

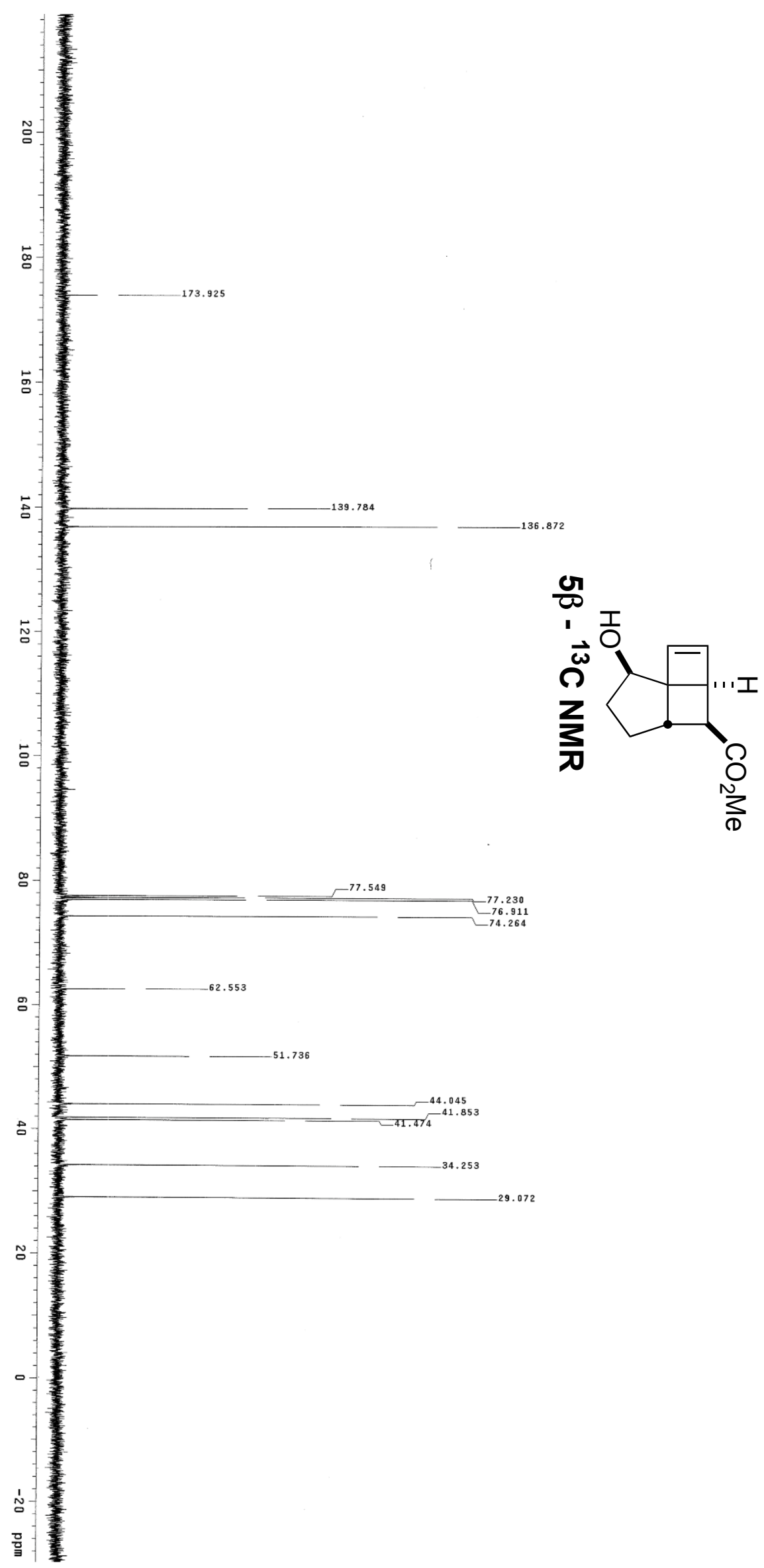


$-S 45$ -

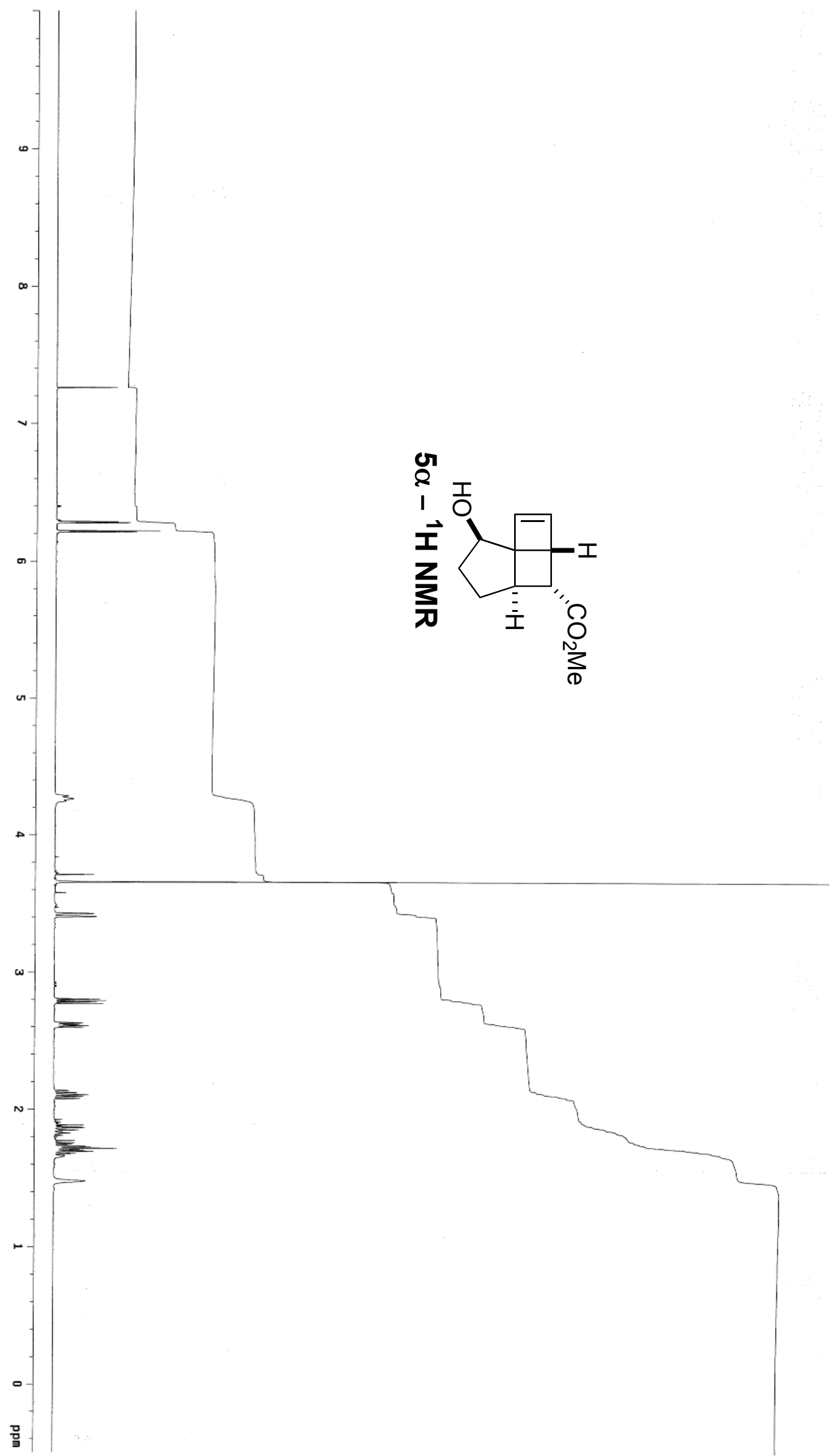


$-S 46-$

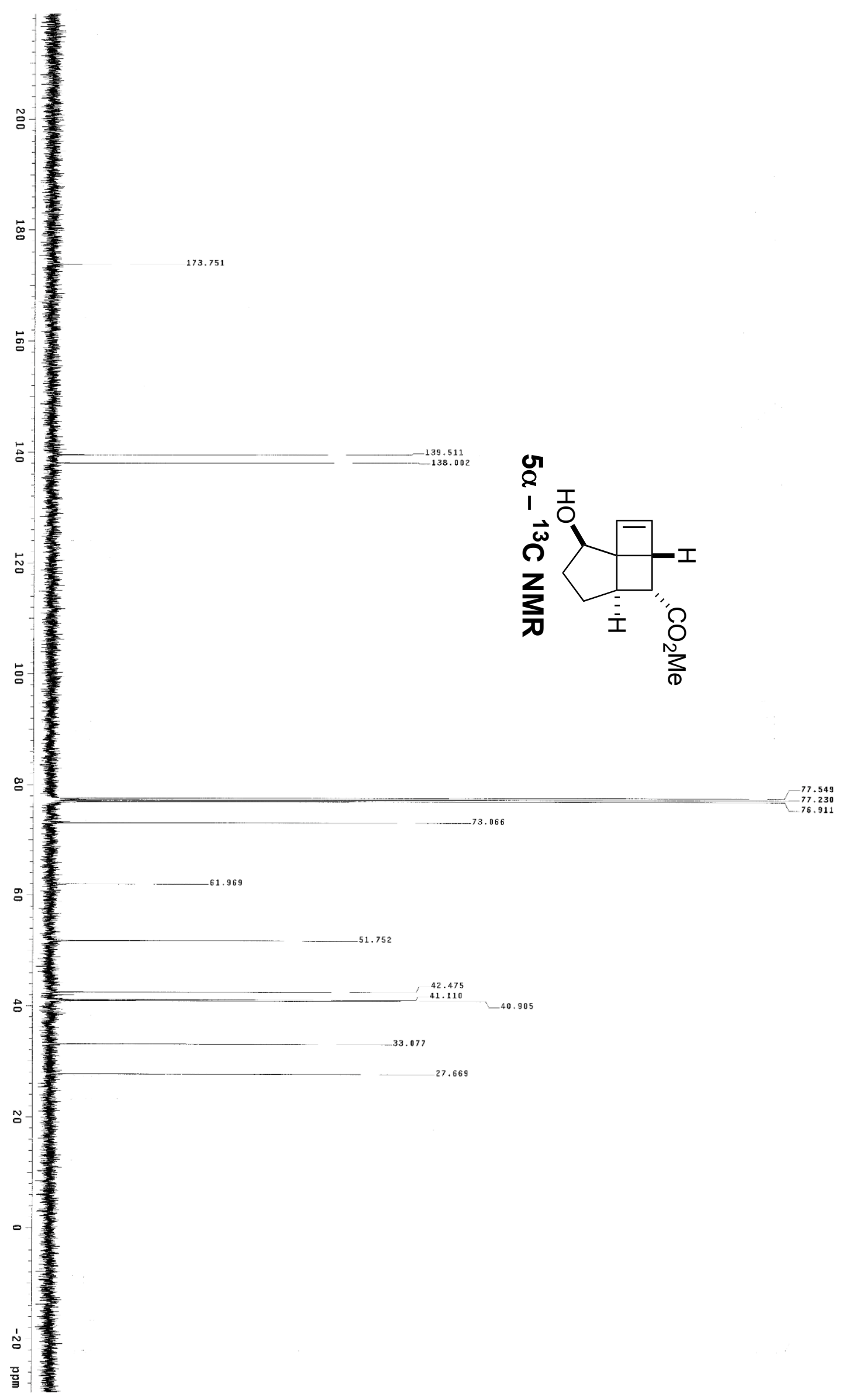


-S 47 -

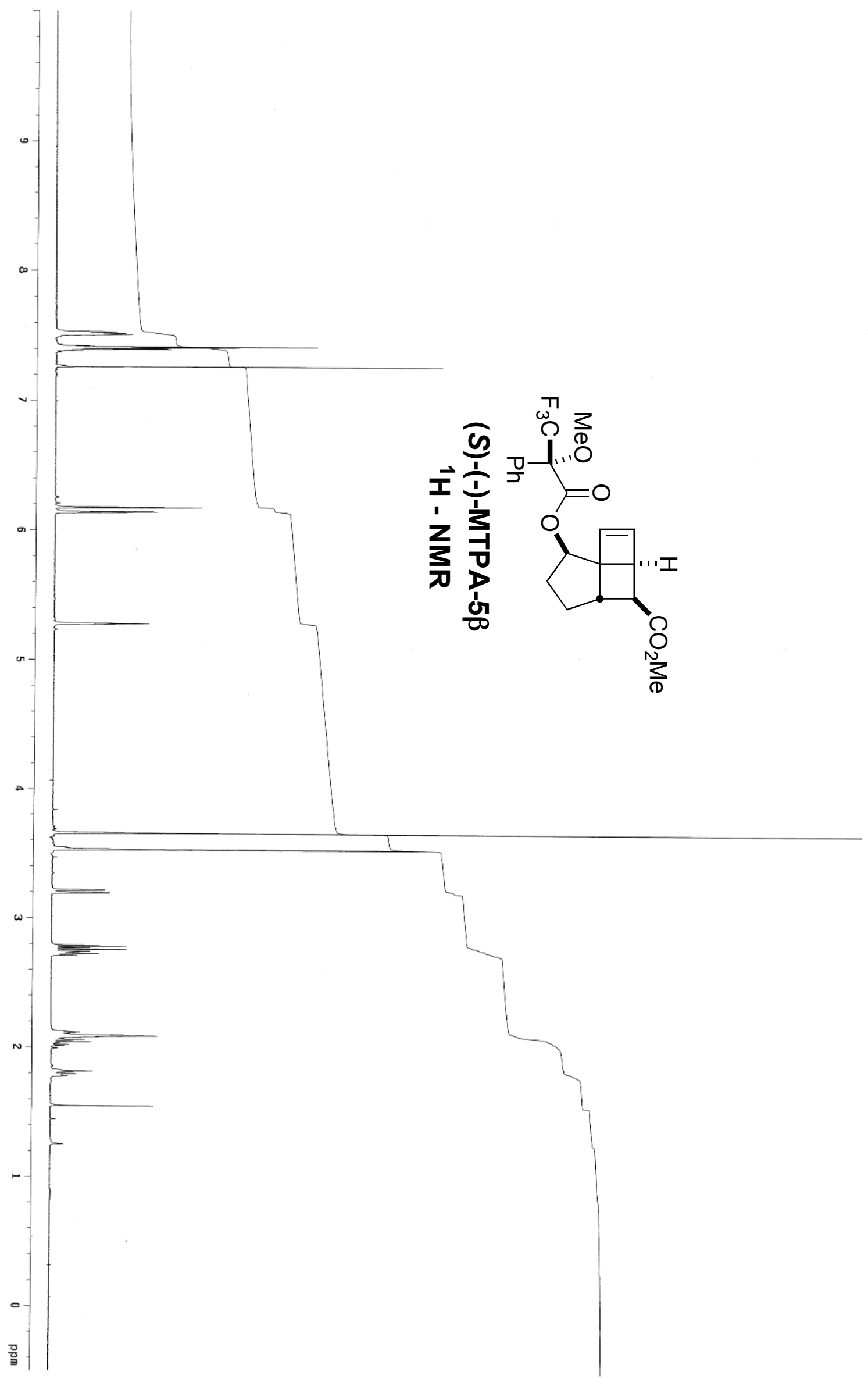




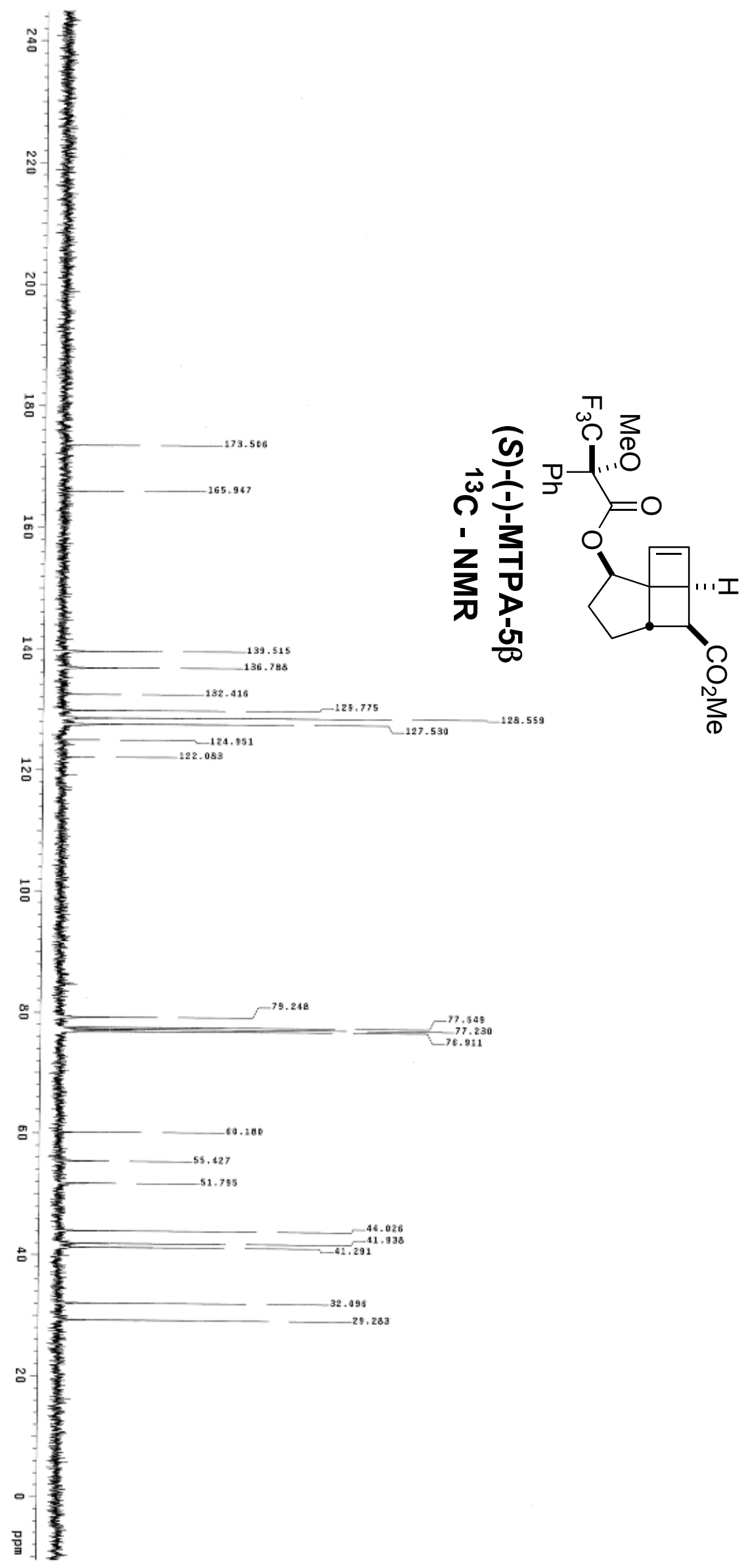




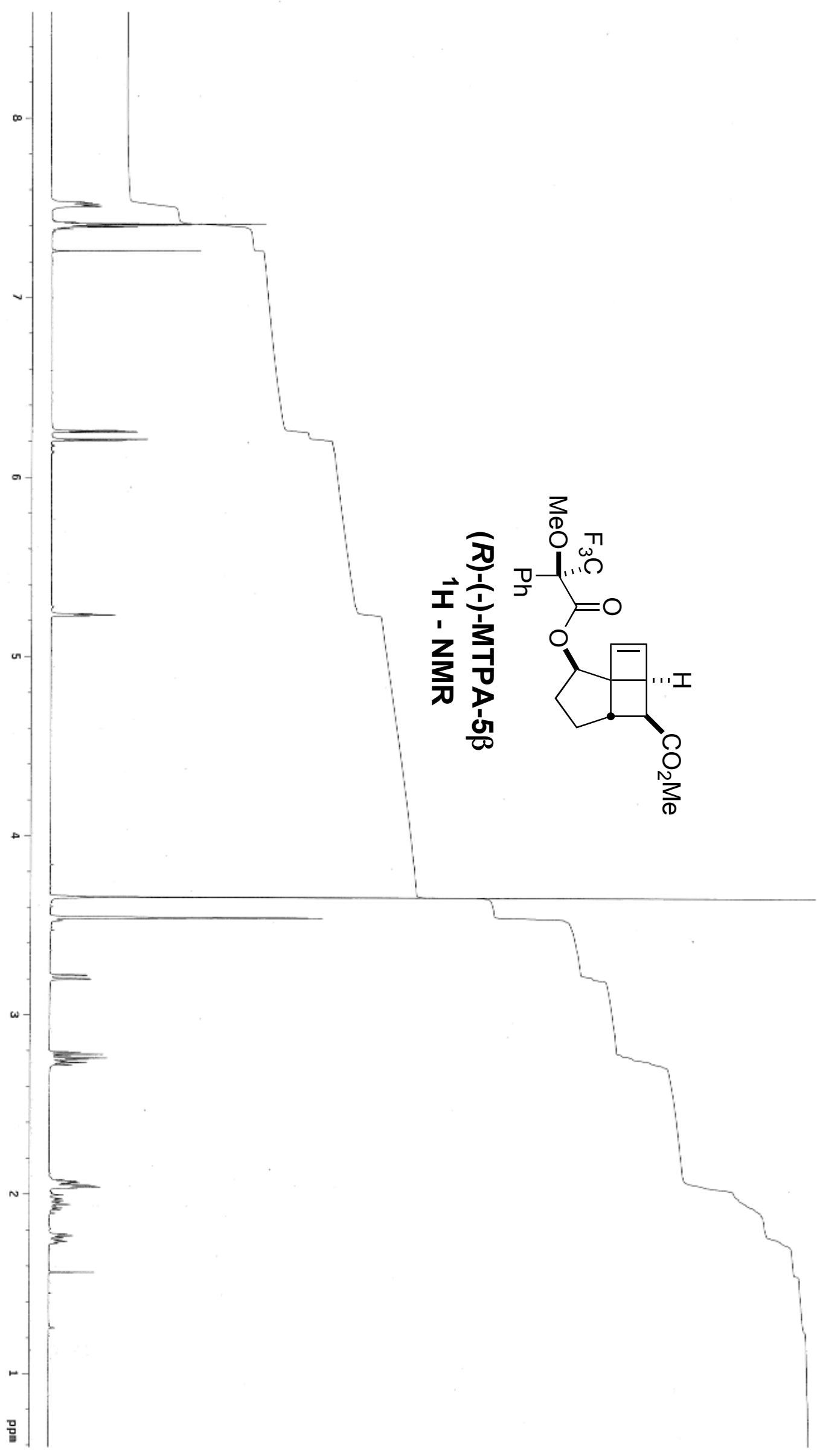


$-S 50$ -

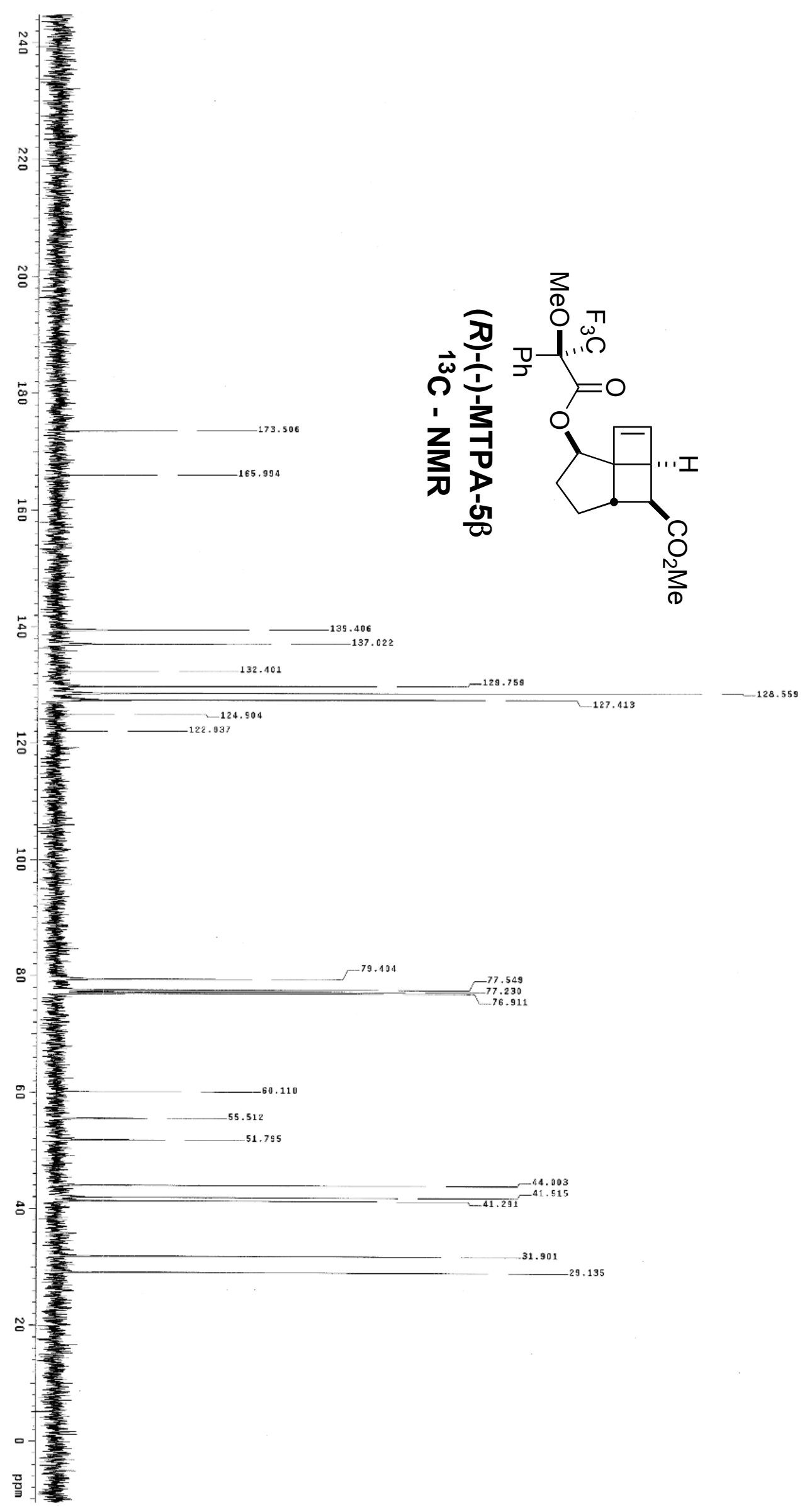


-S $51-$

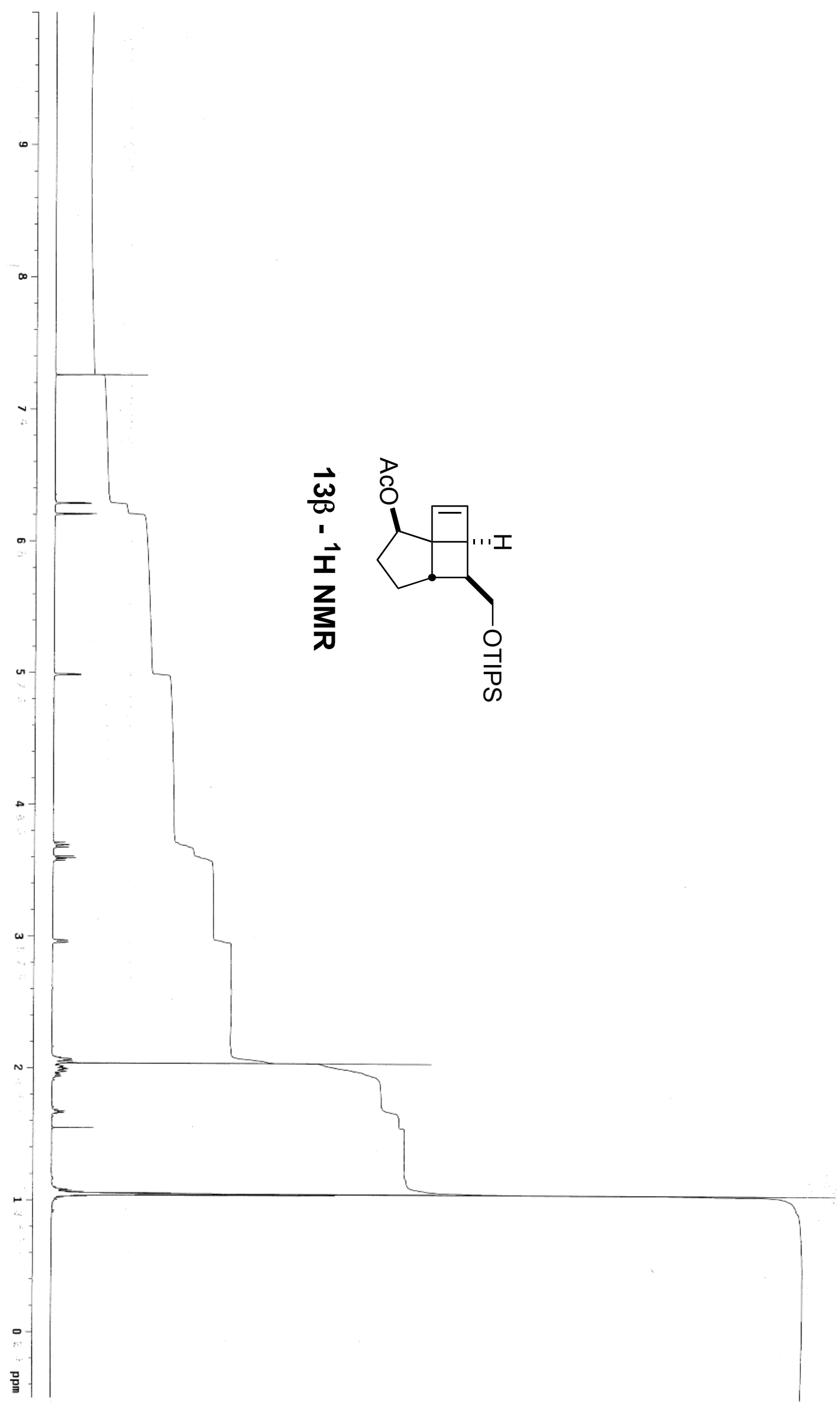


$-S 52-$

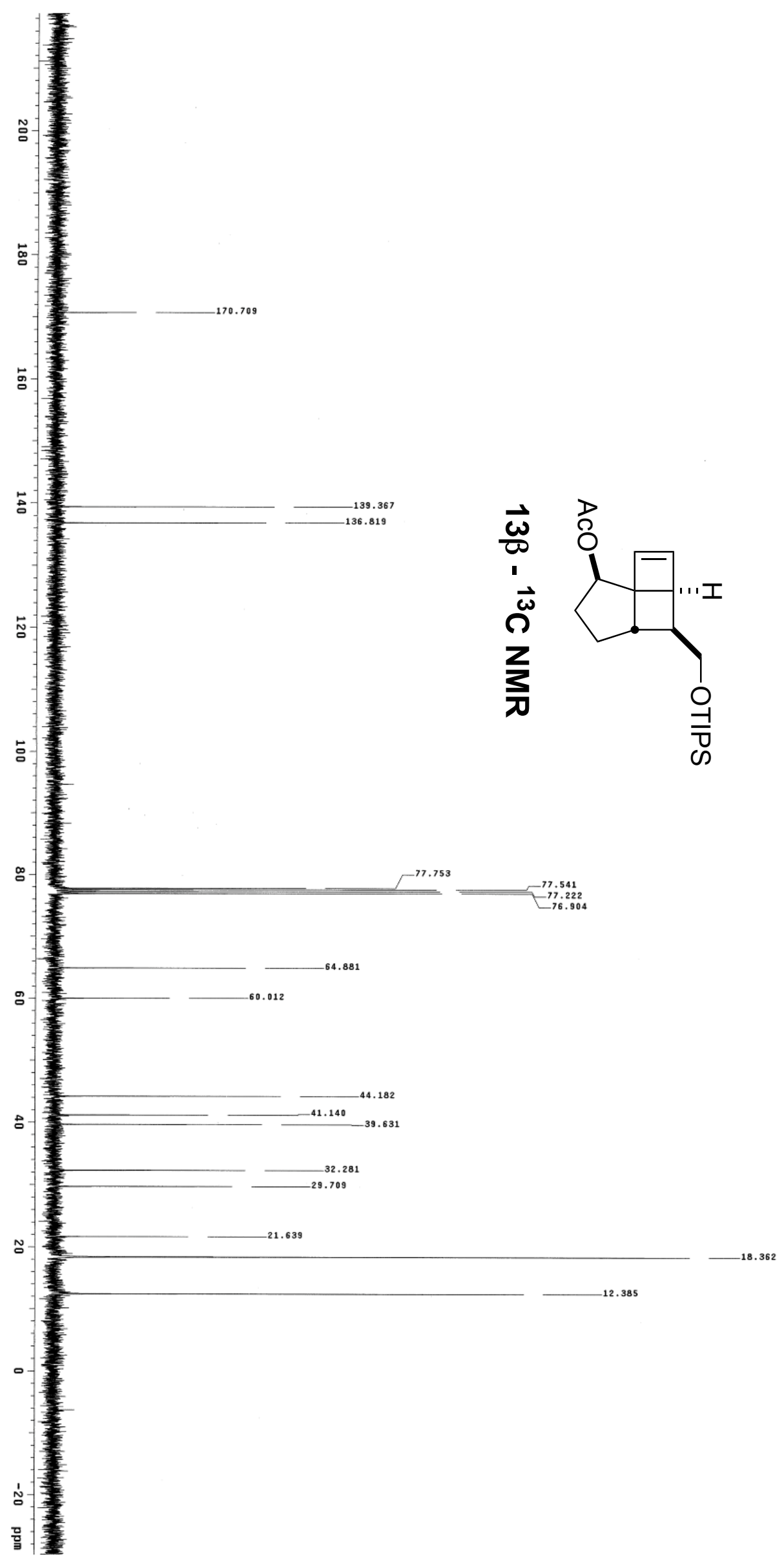


$-S 53-$

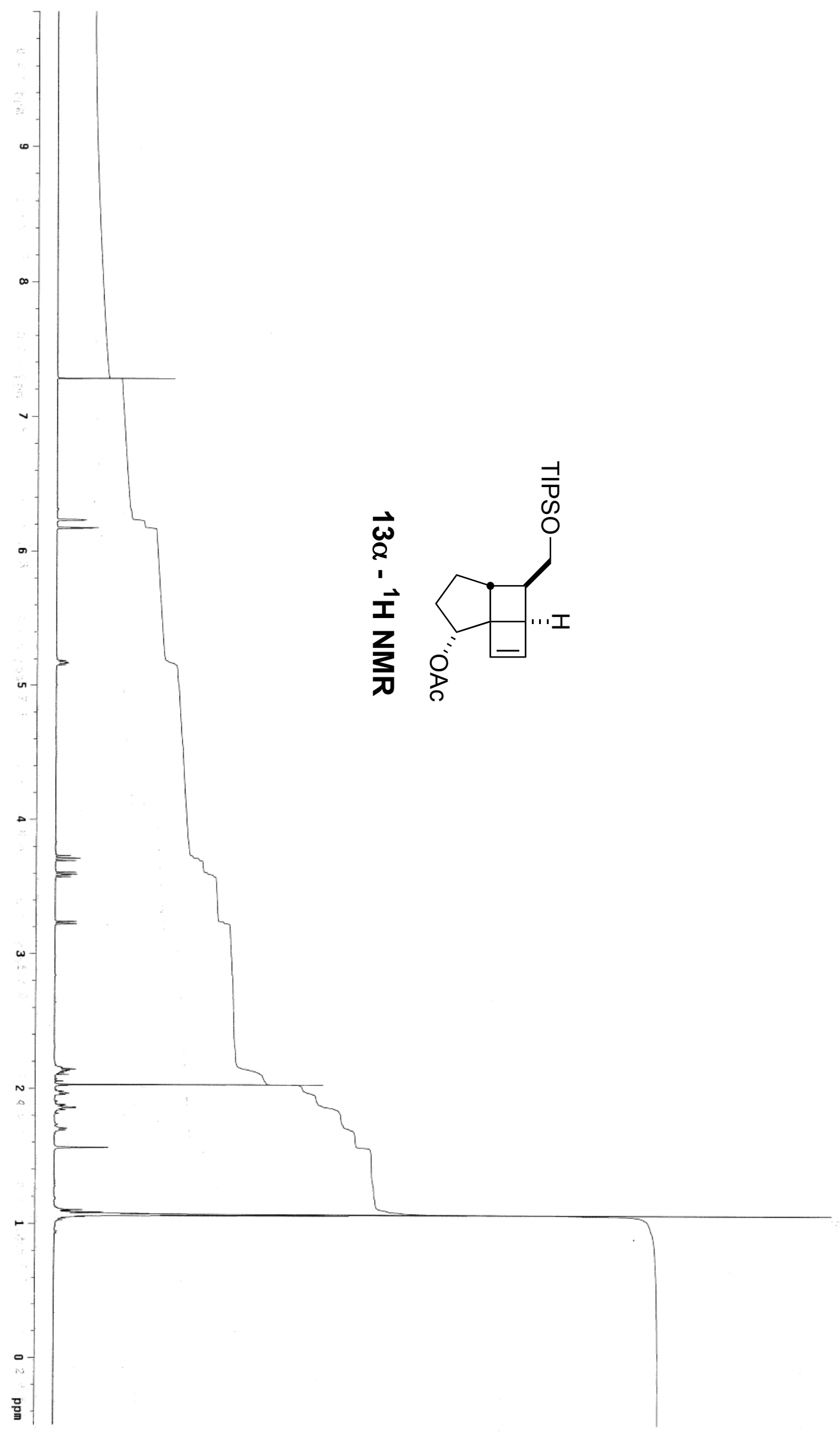




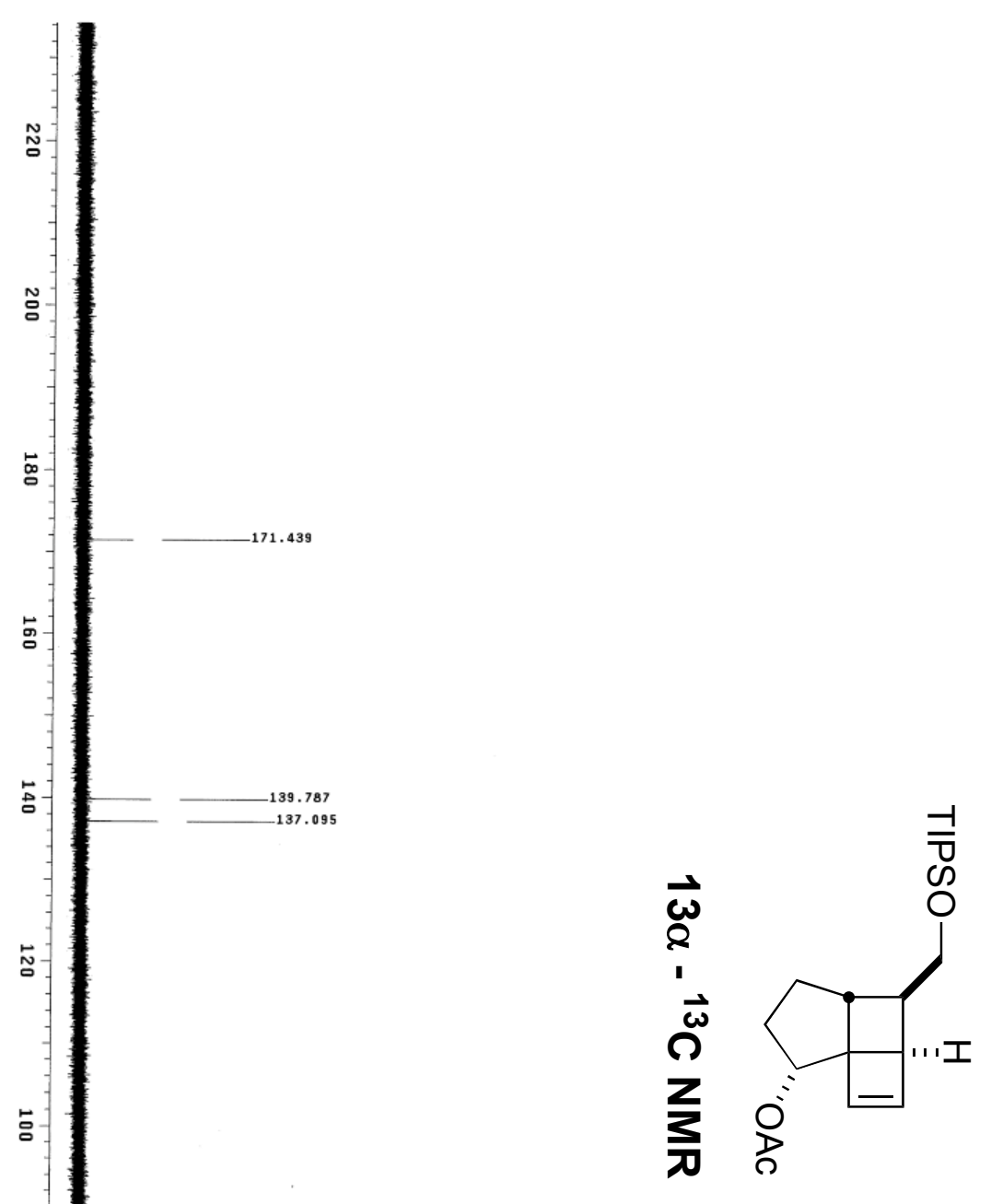


$-S 55$ -

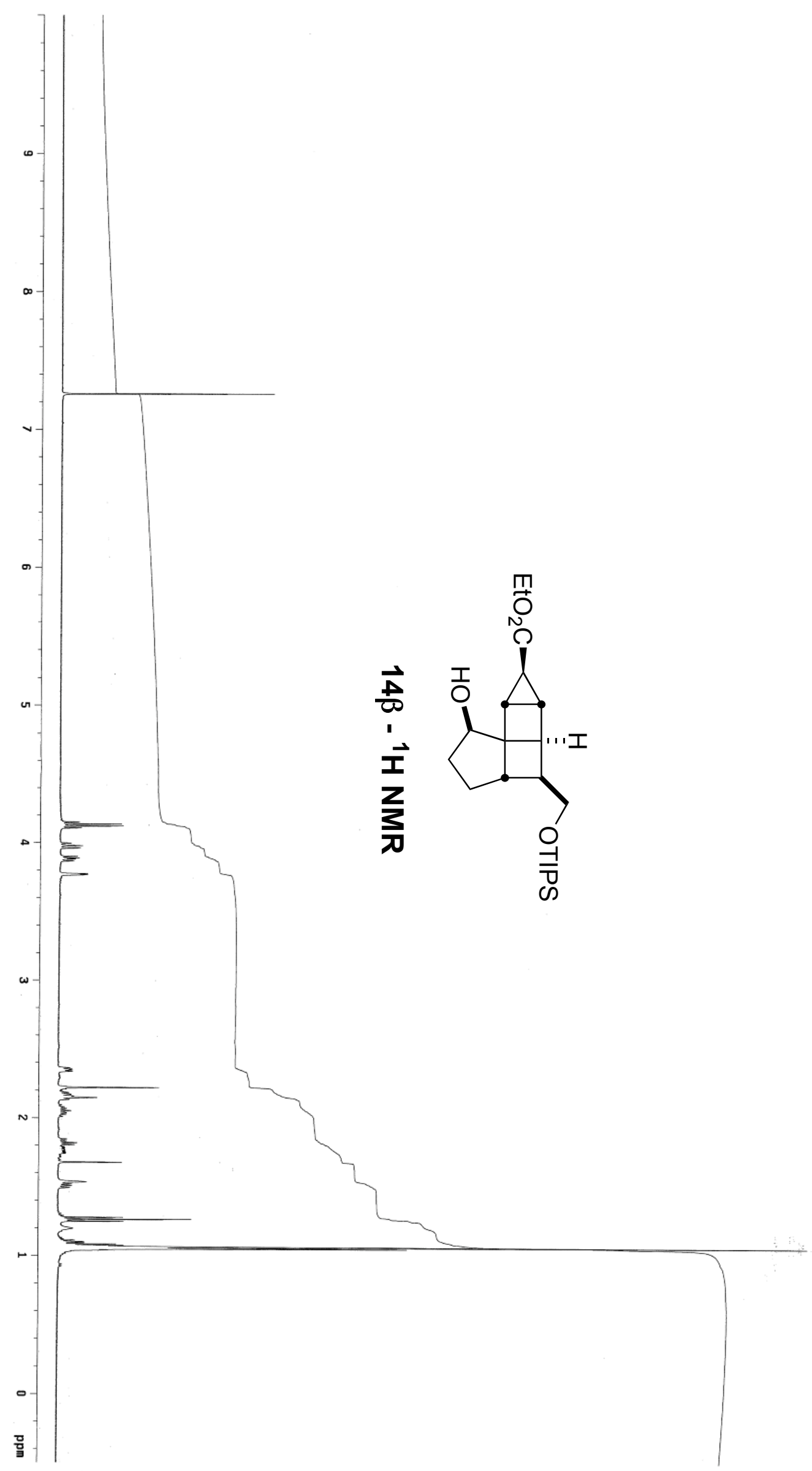




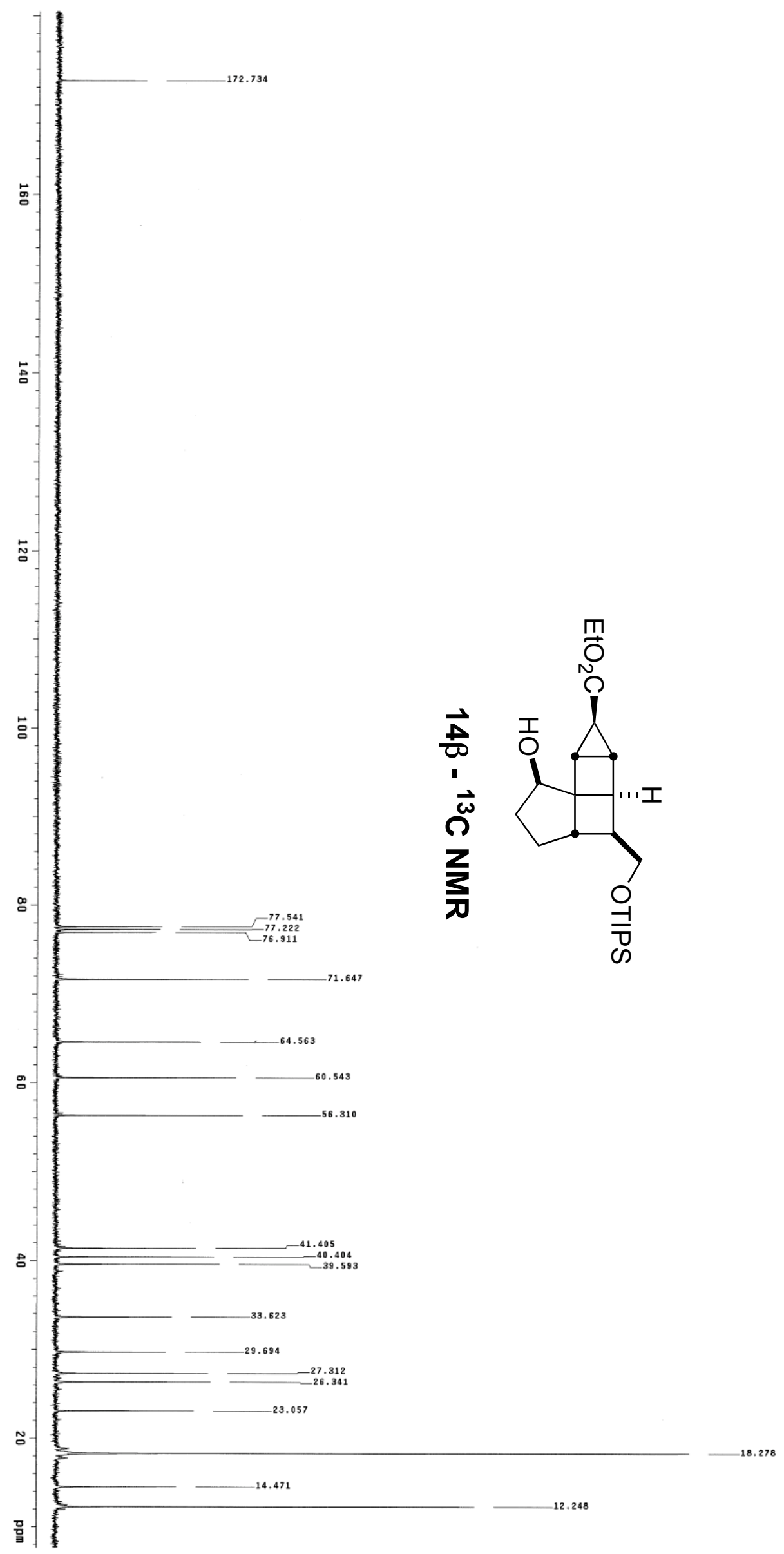


-S 57 -

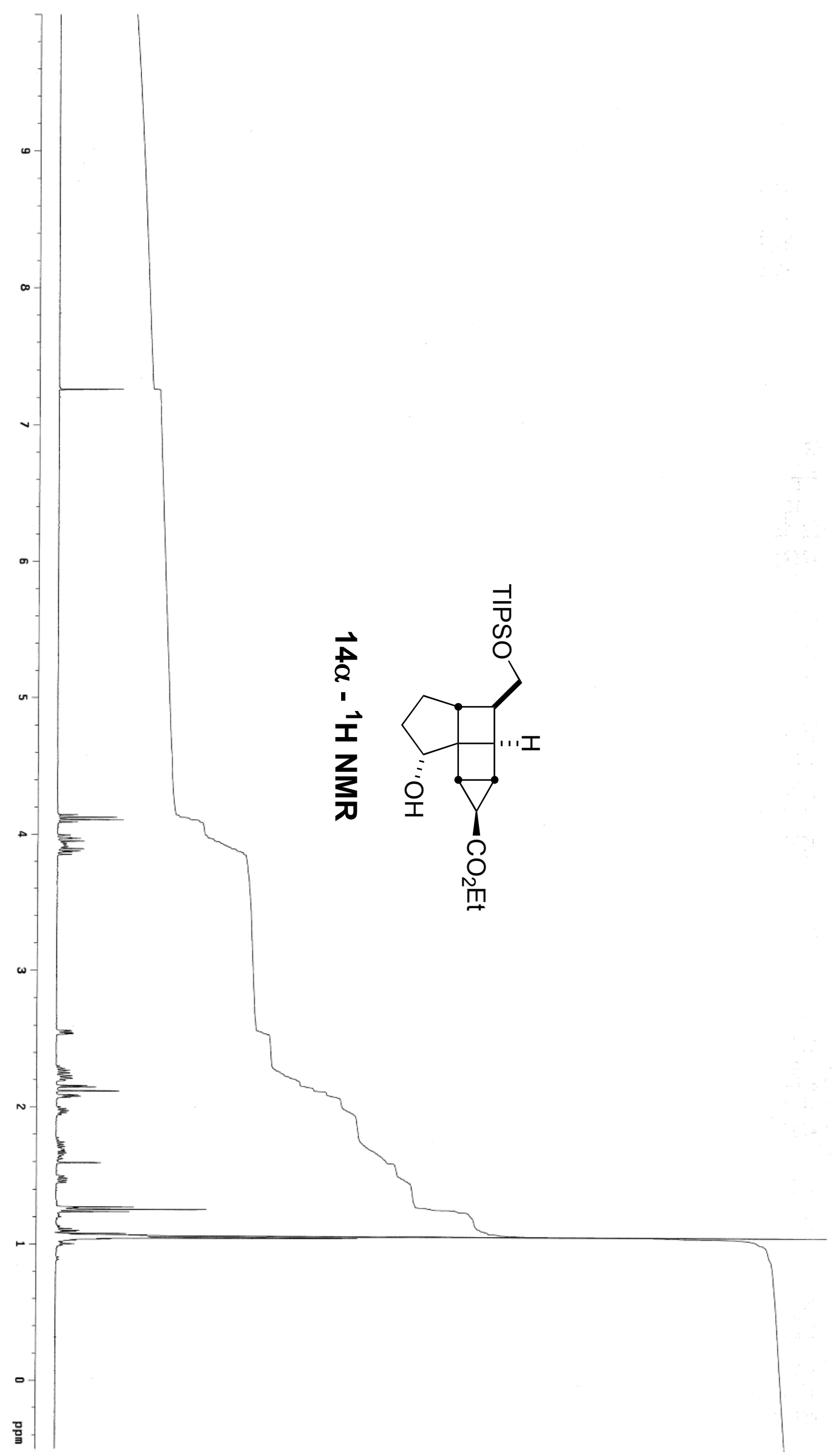




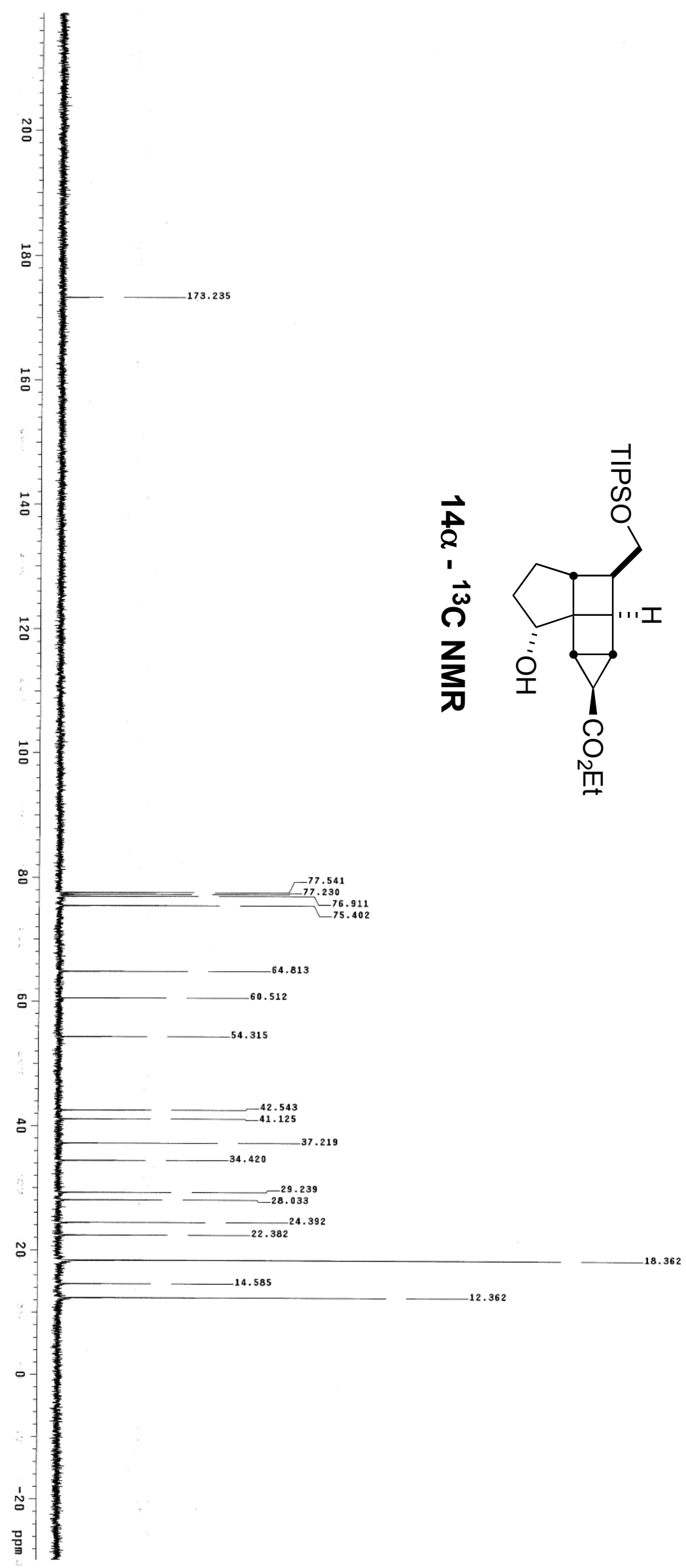




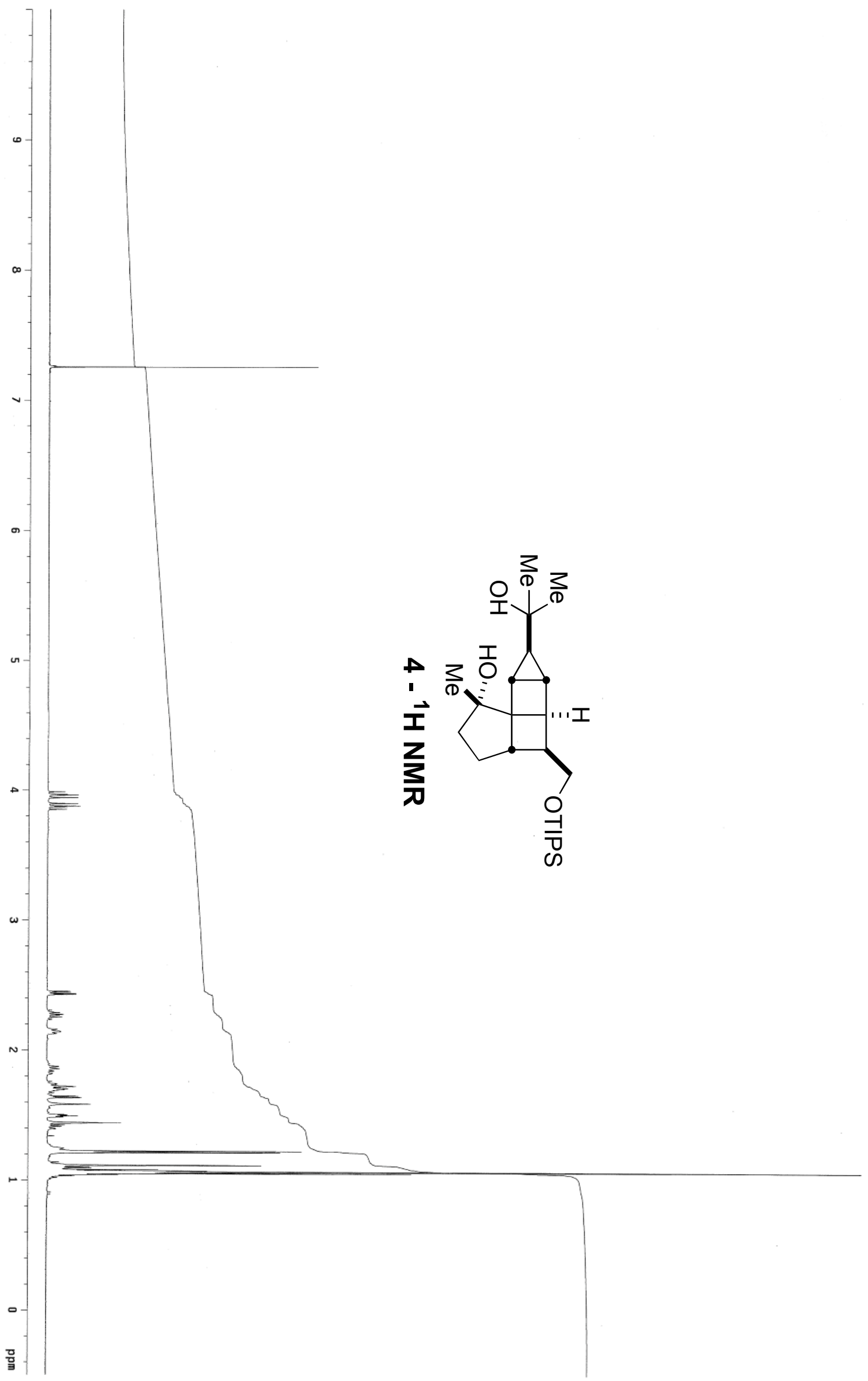




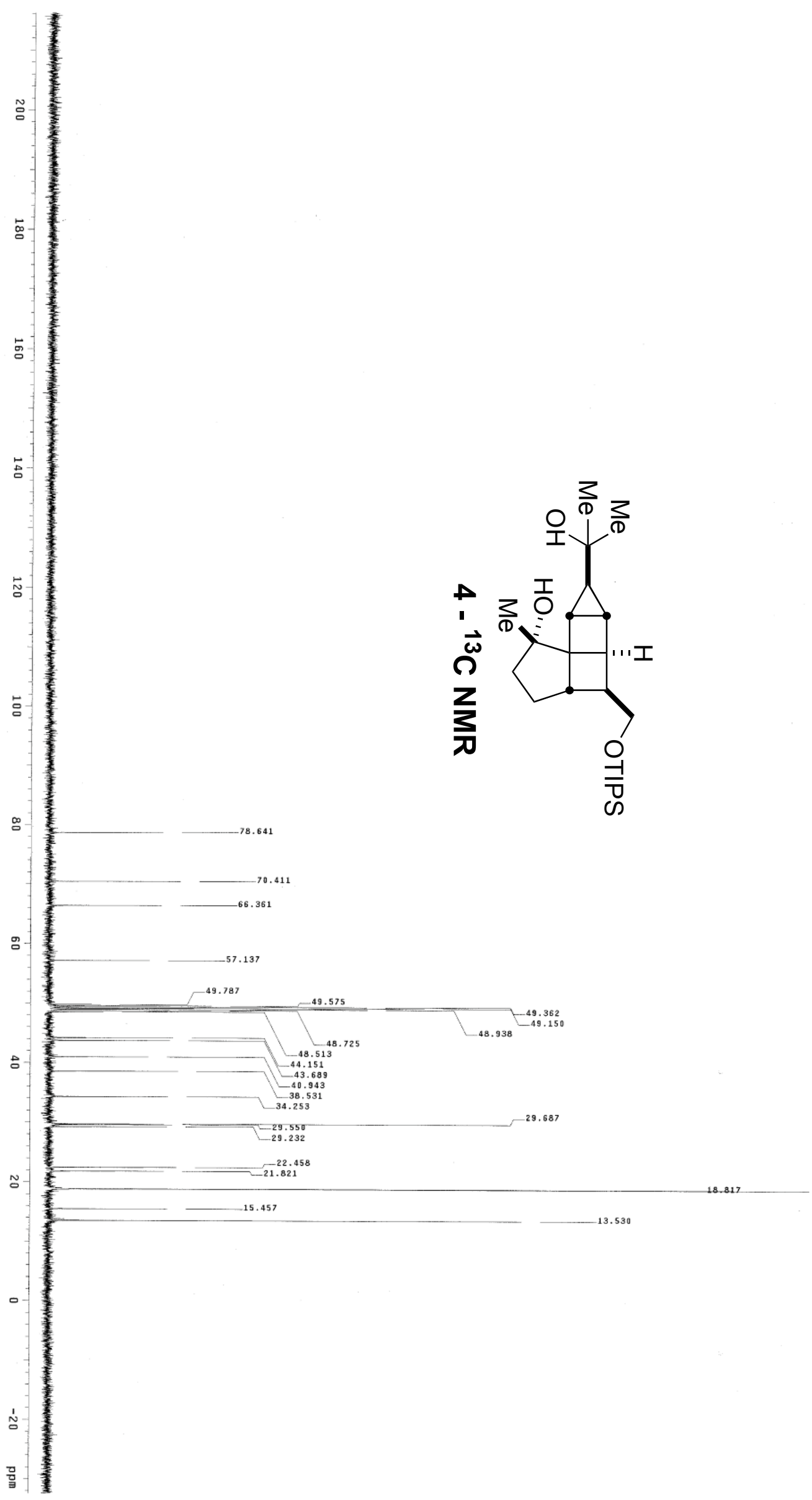


$-S 61-$

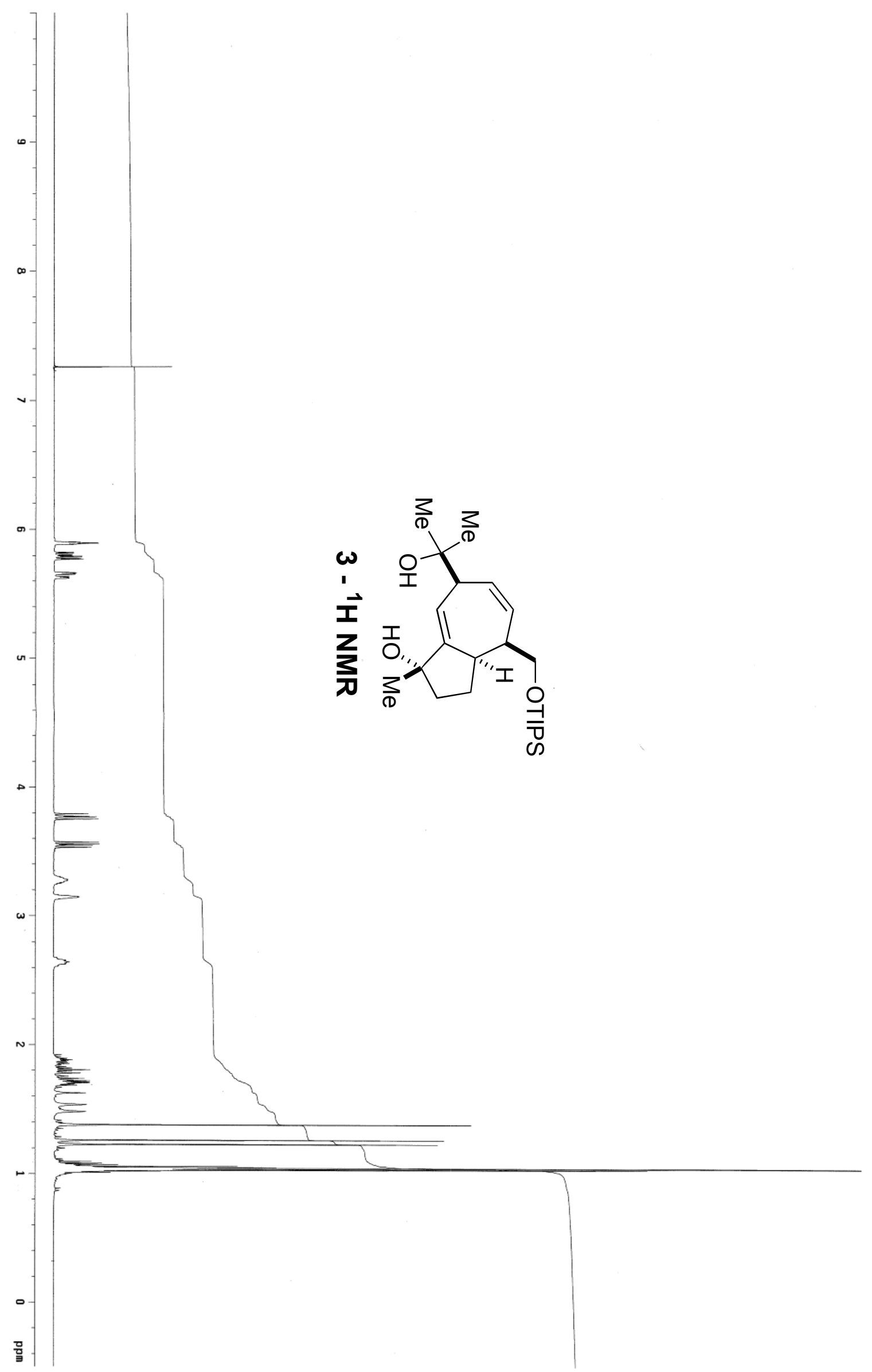




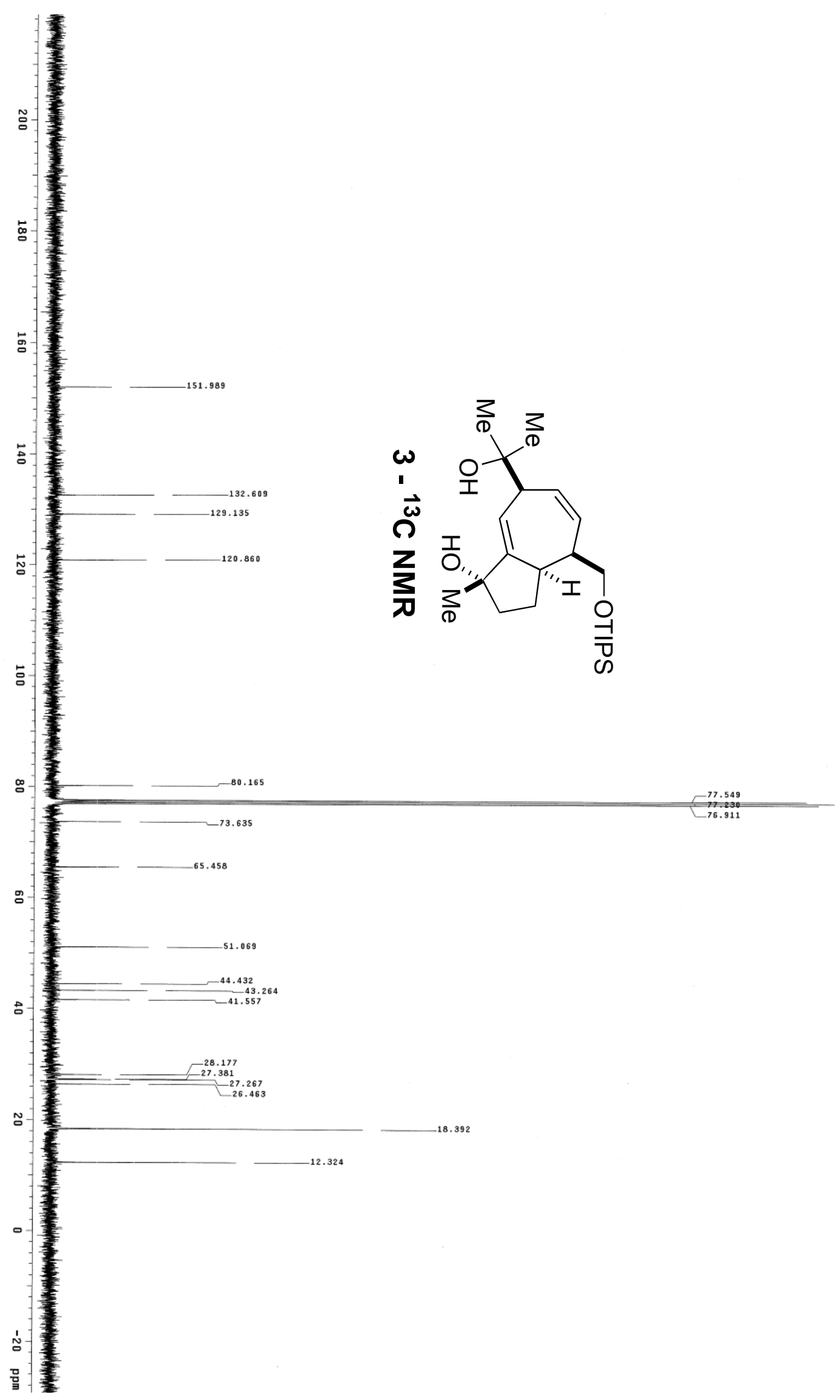


$-S 63-$

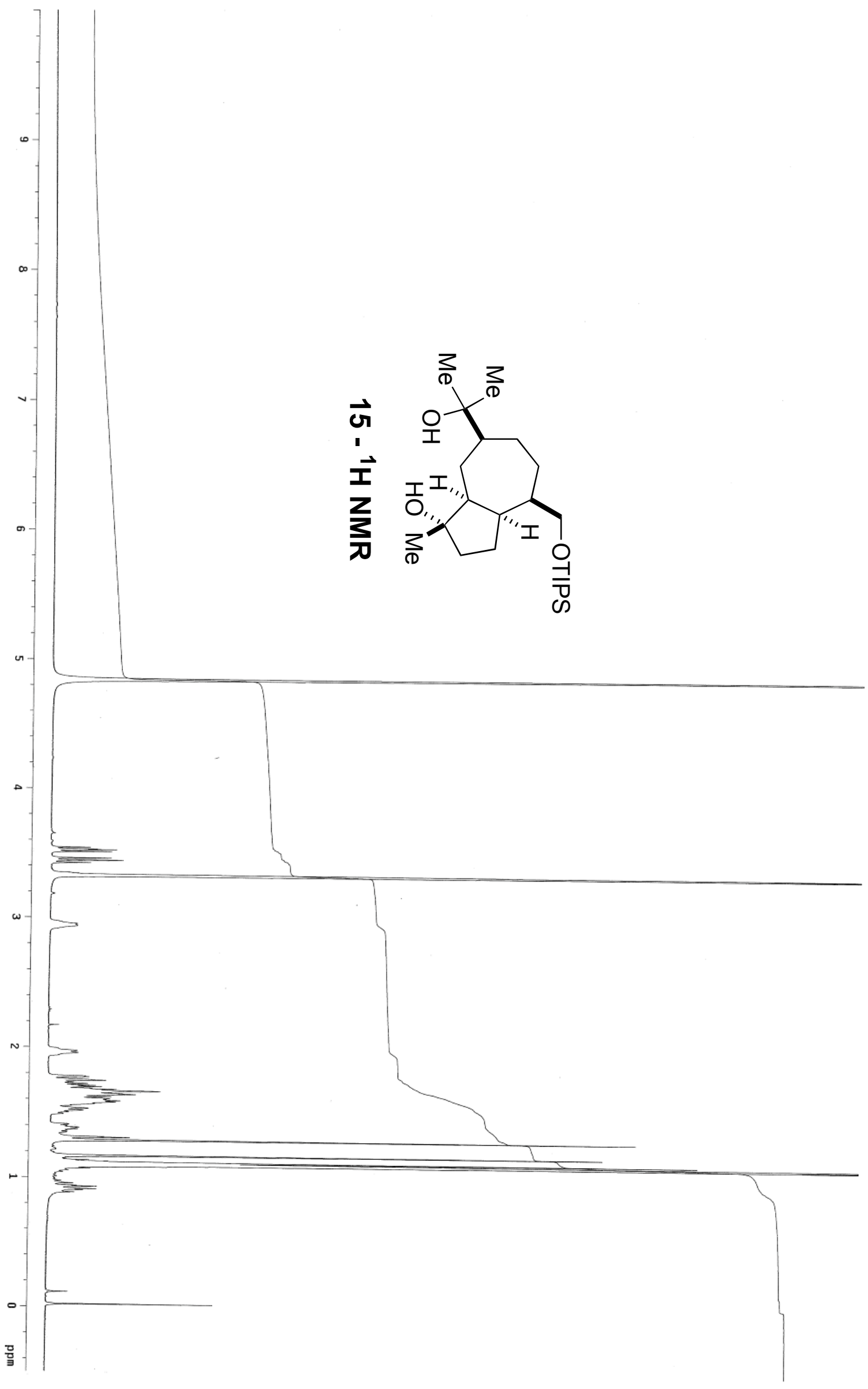




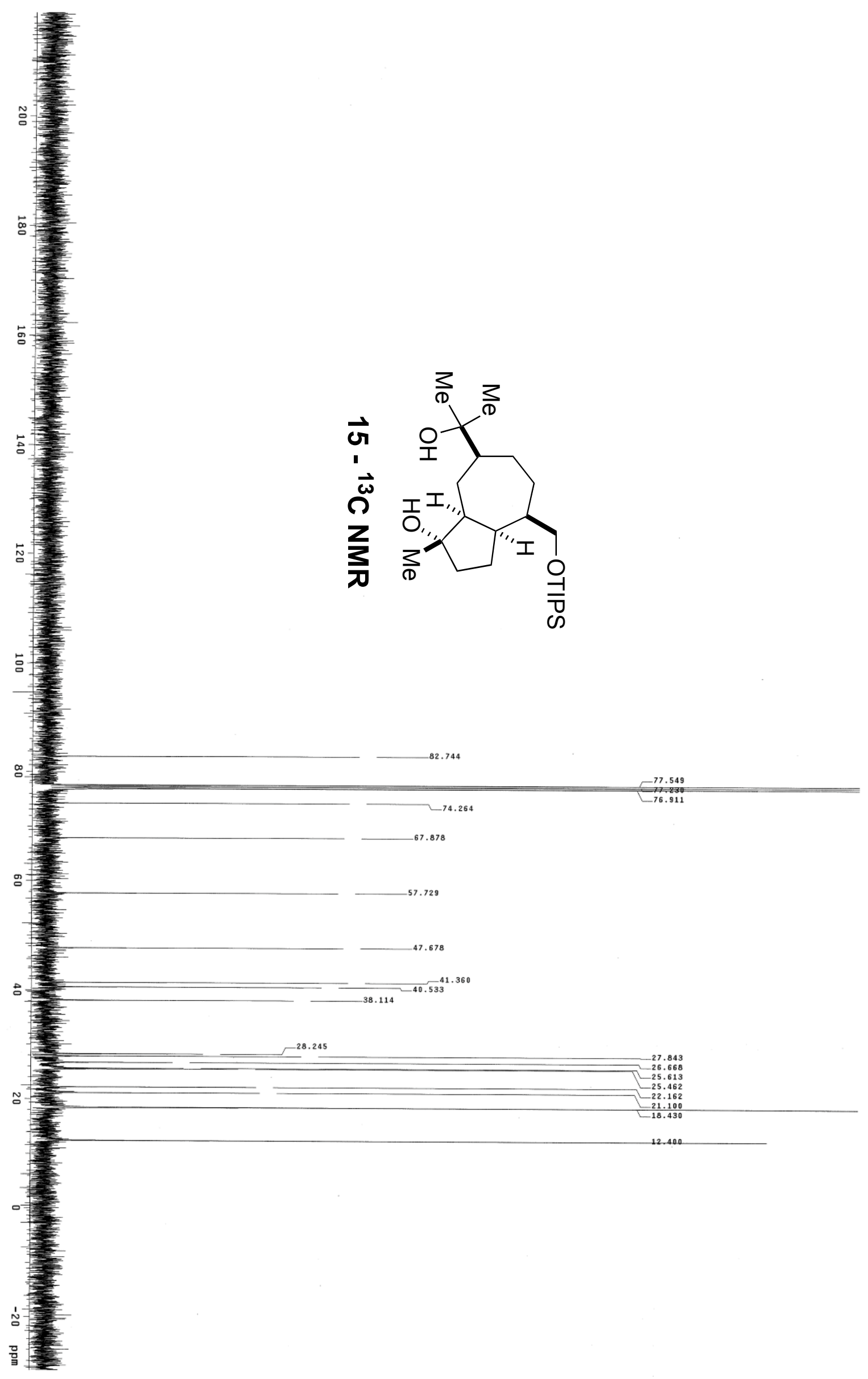


$-S 65-$

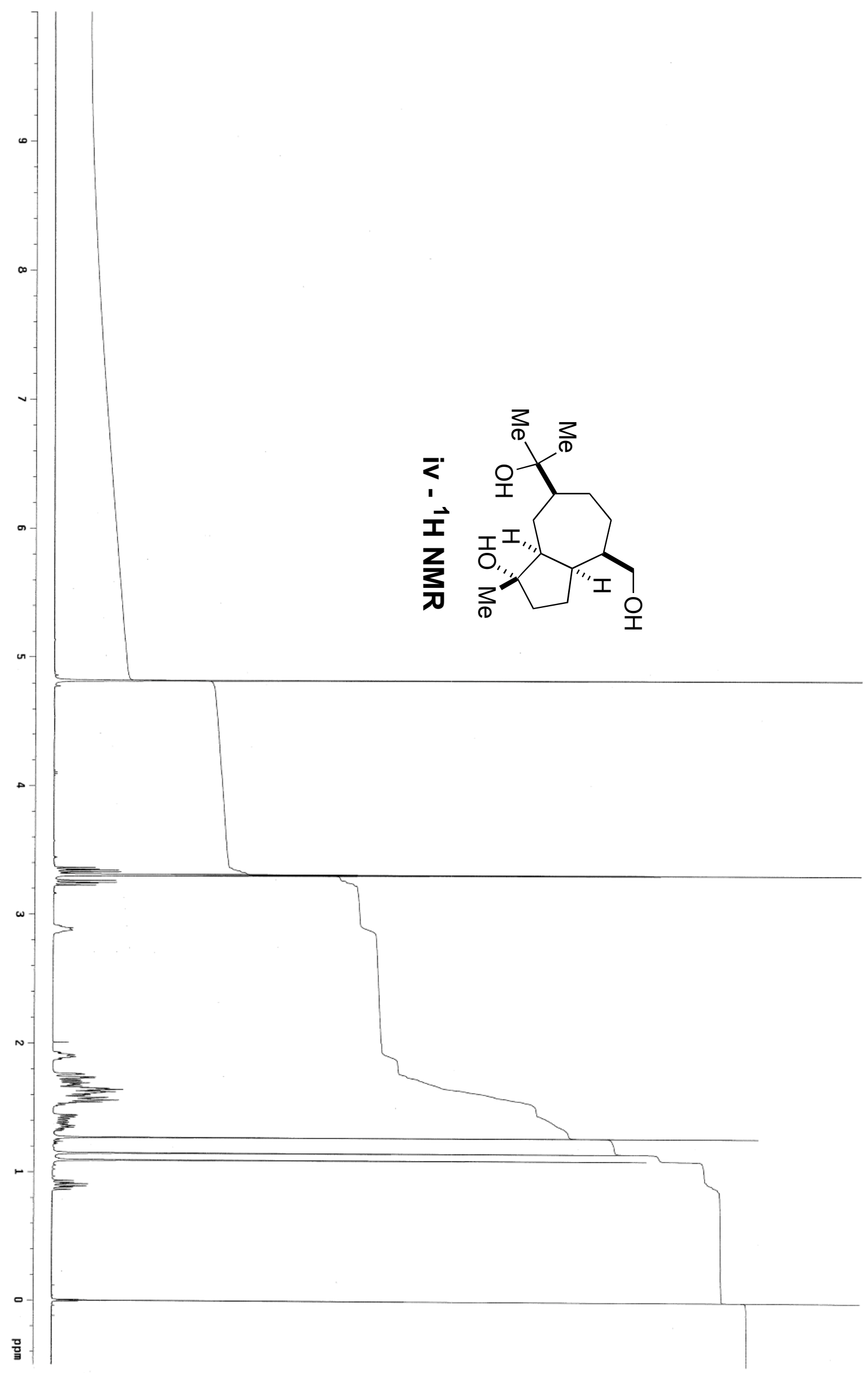




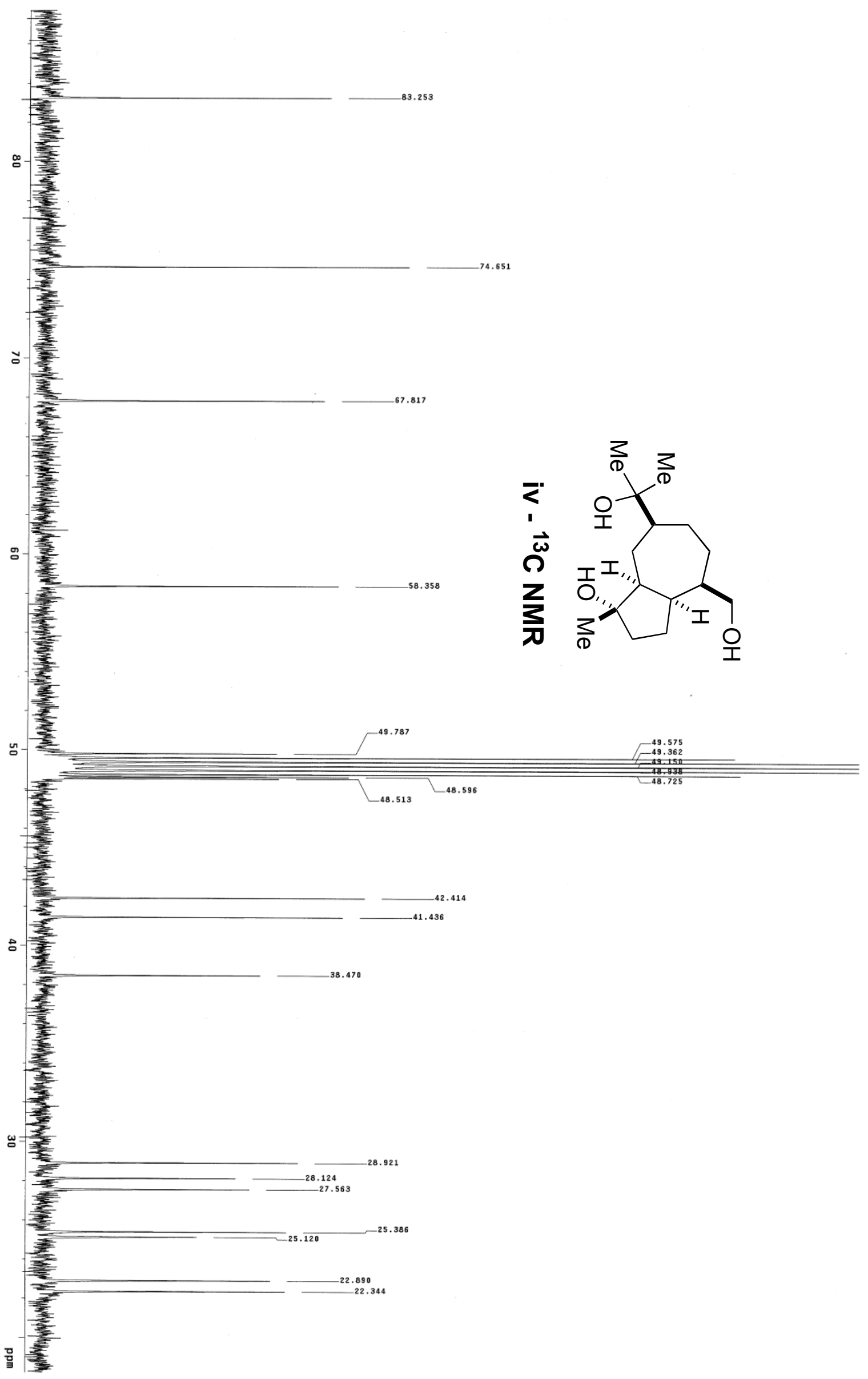


$-S 67$ -

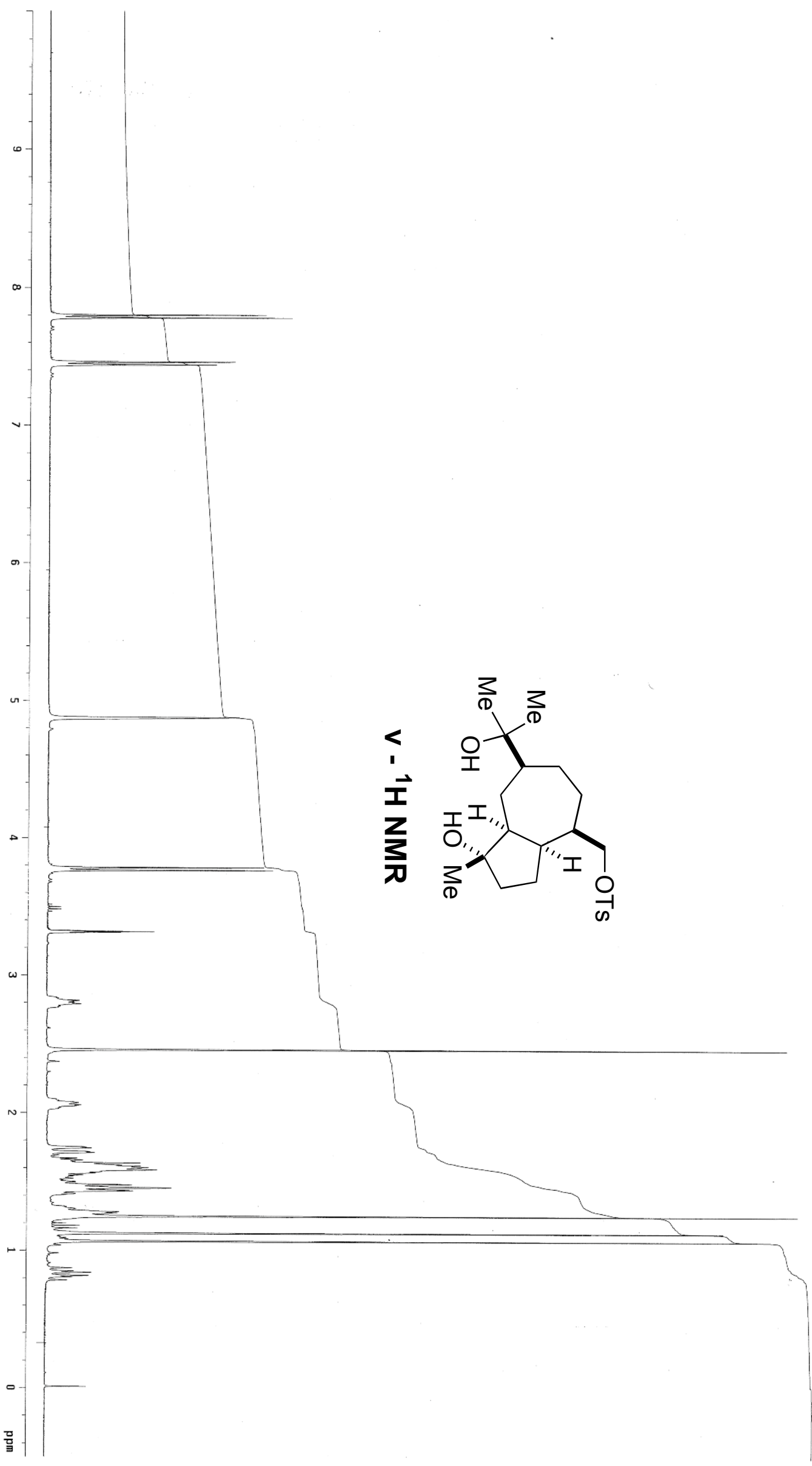




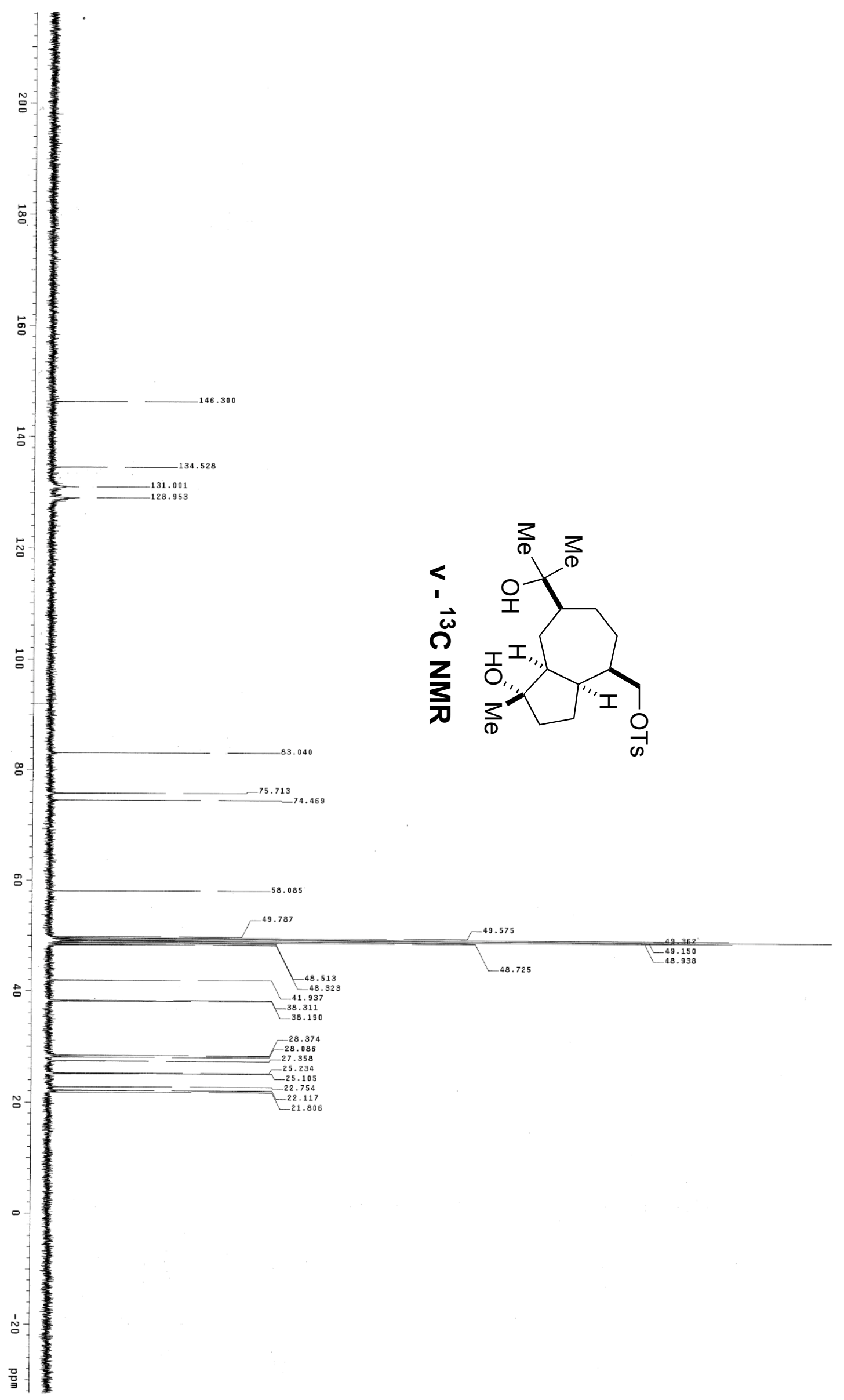




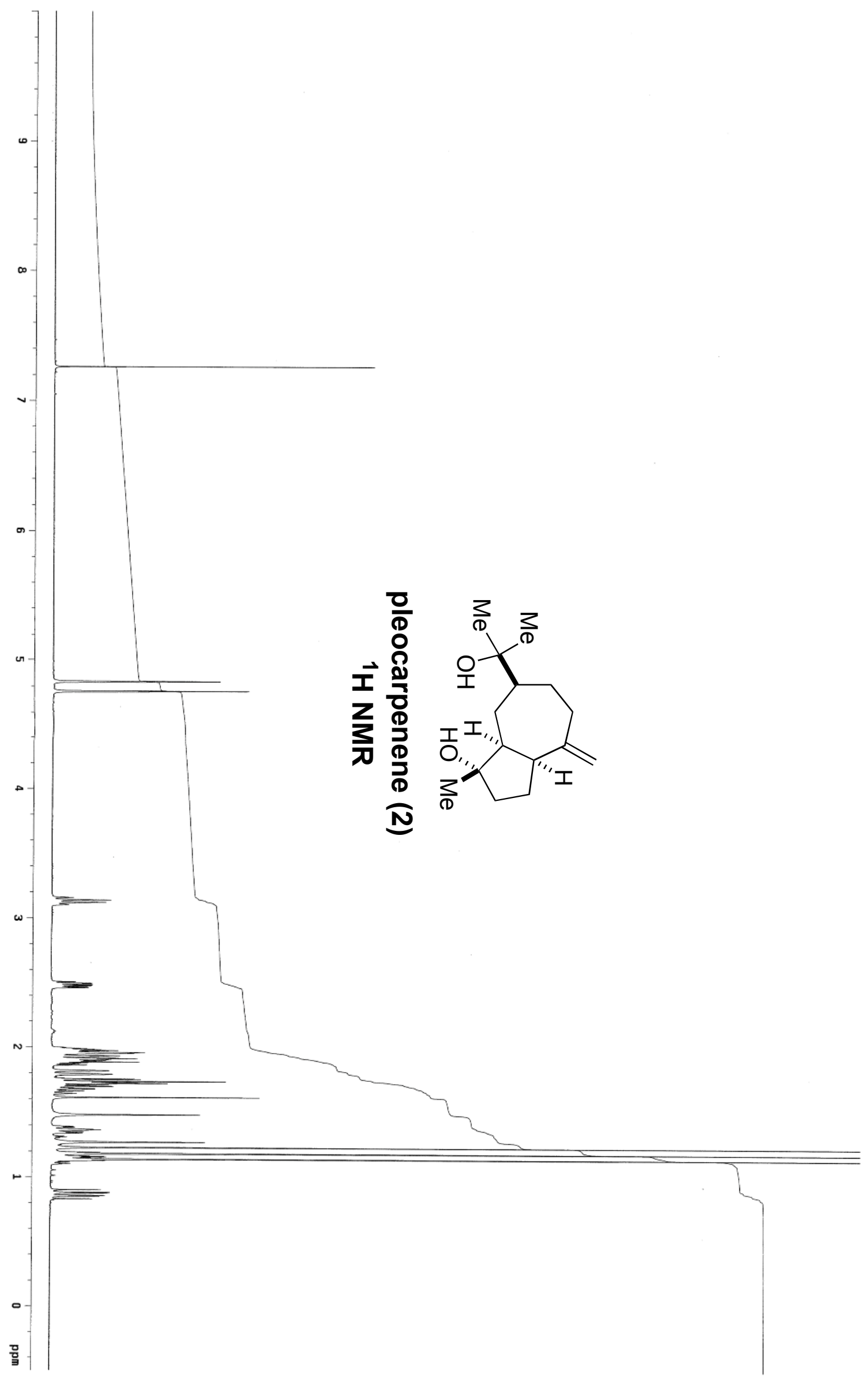



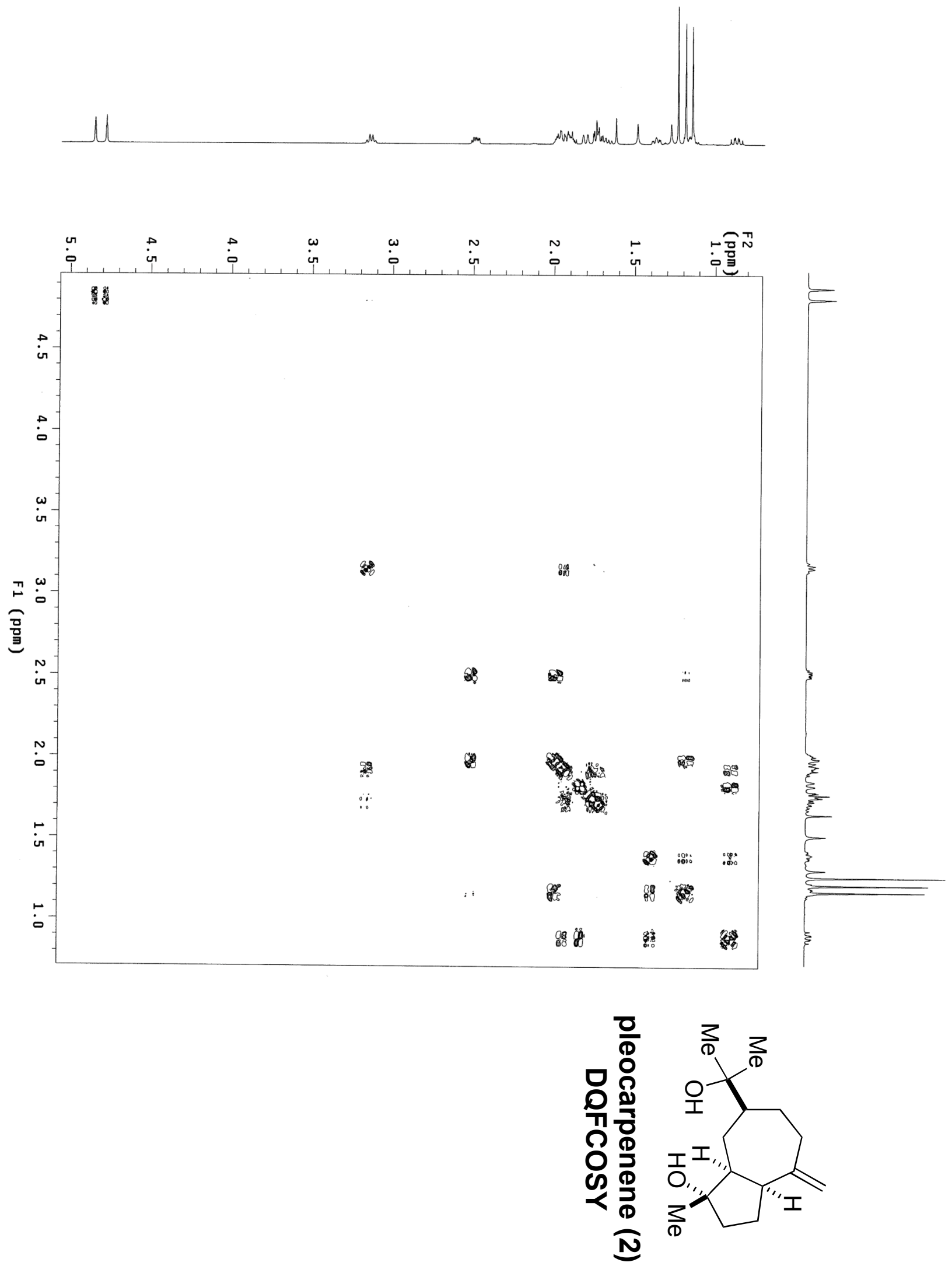

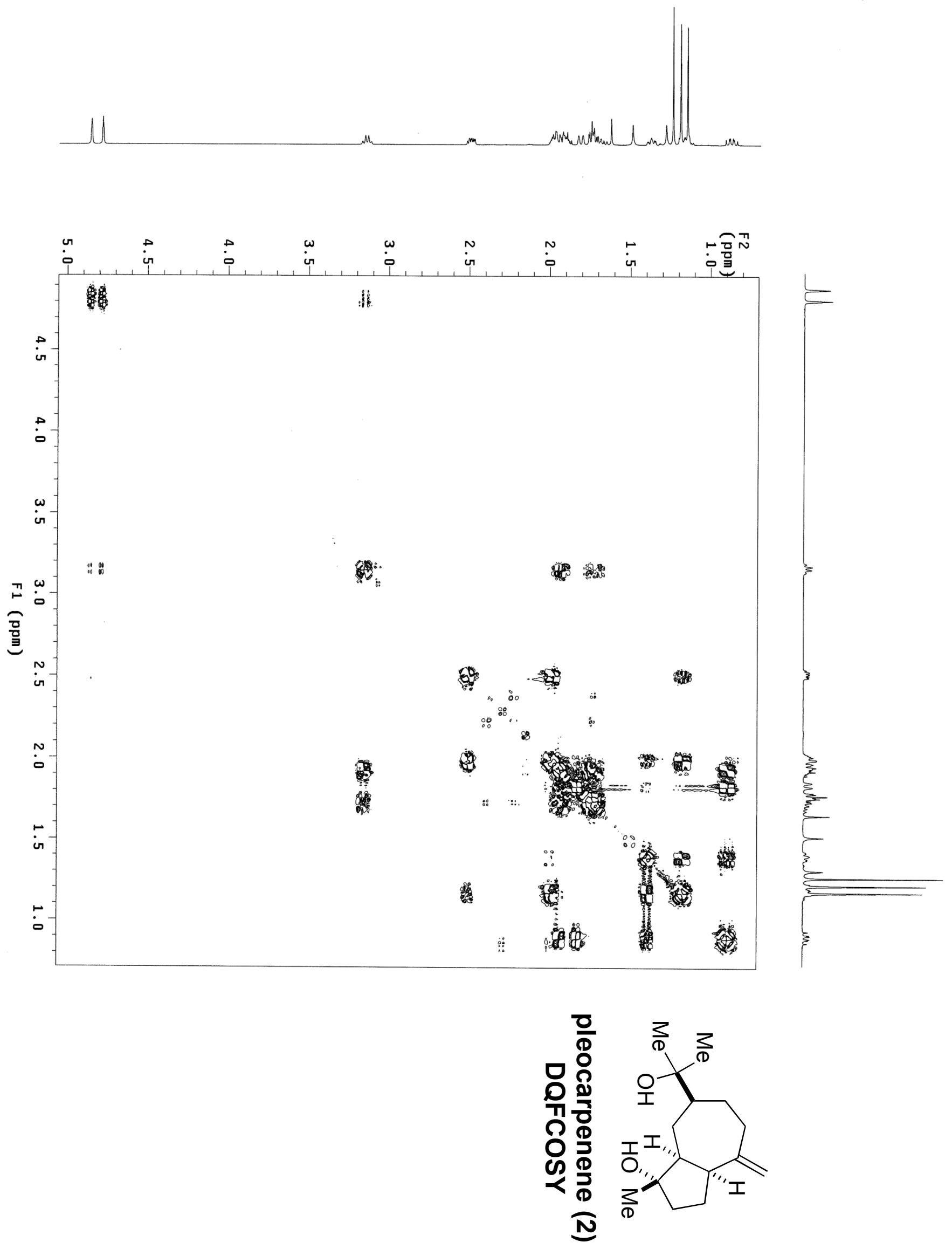


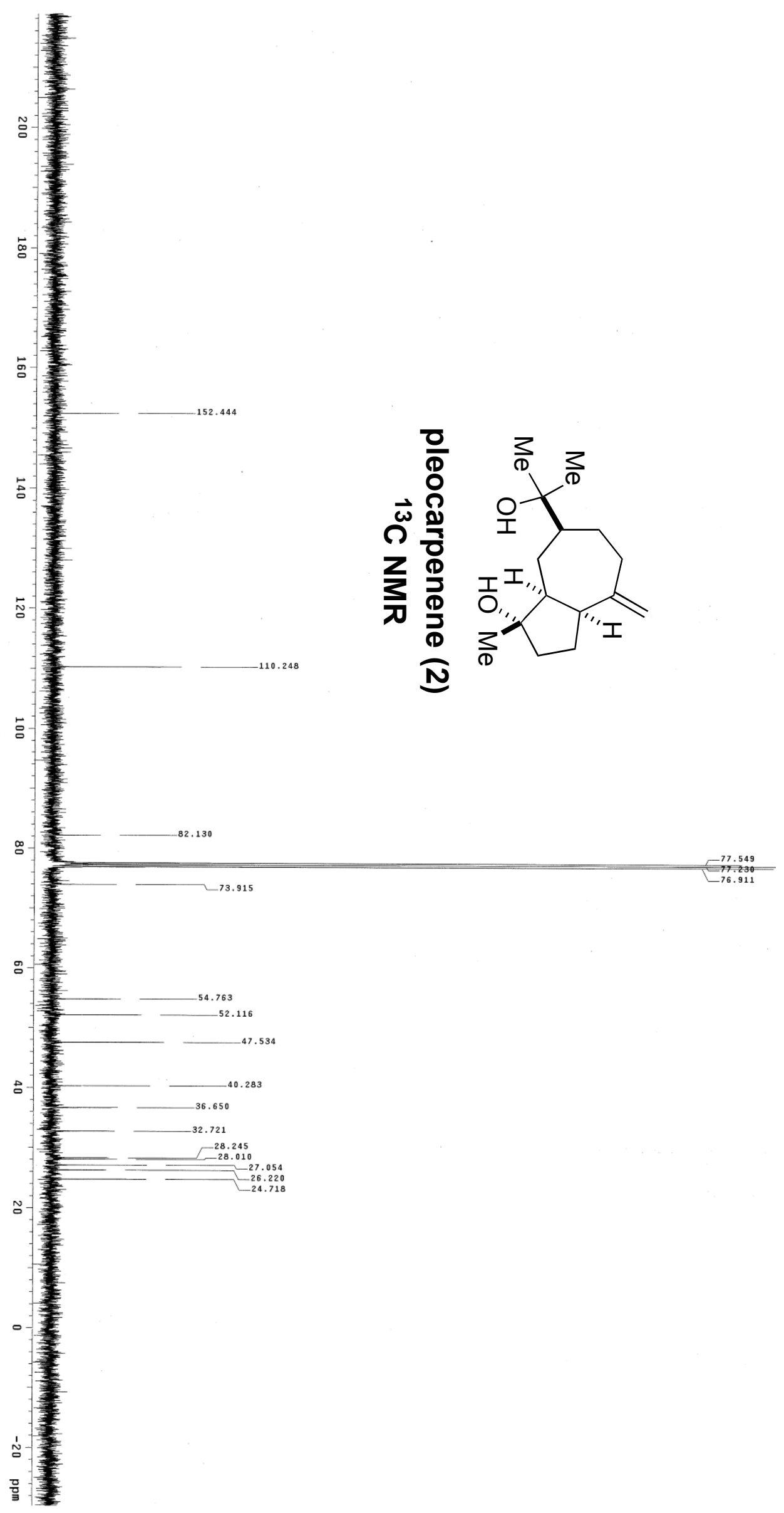


$-S 73-$

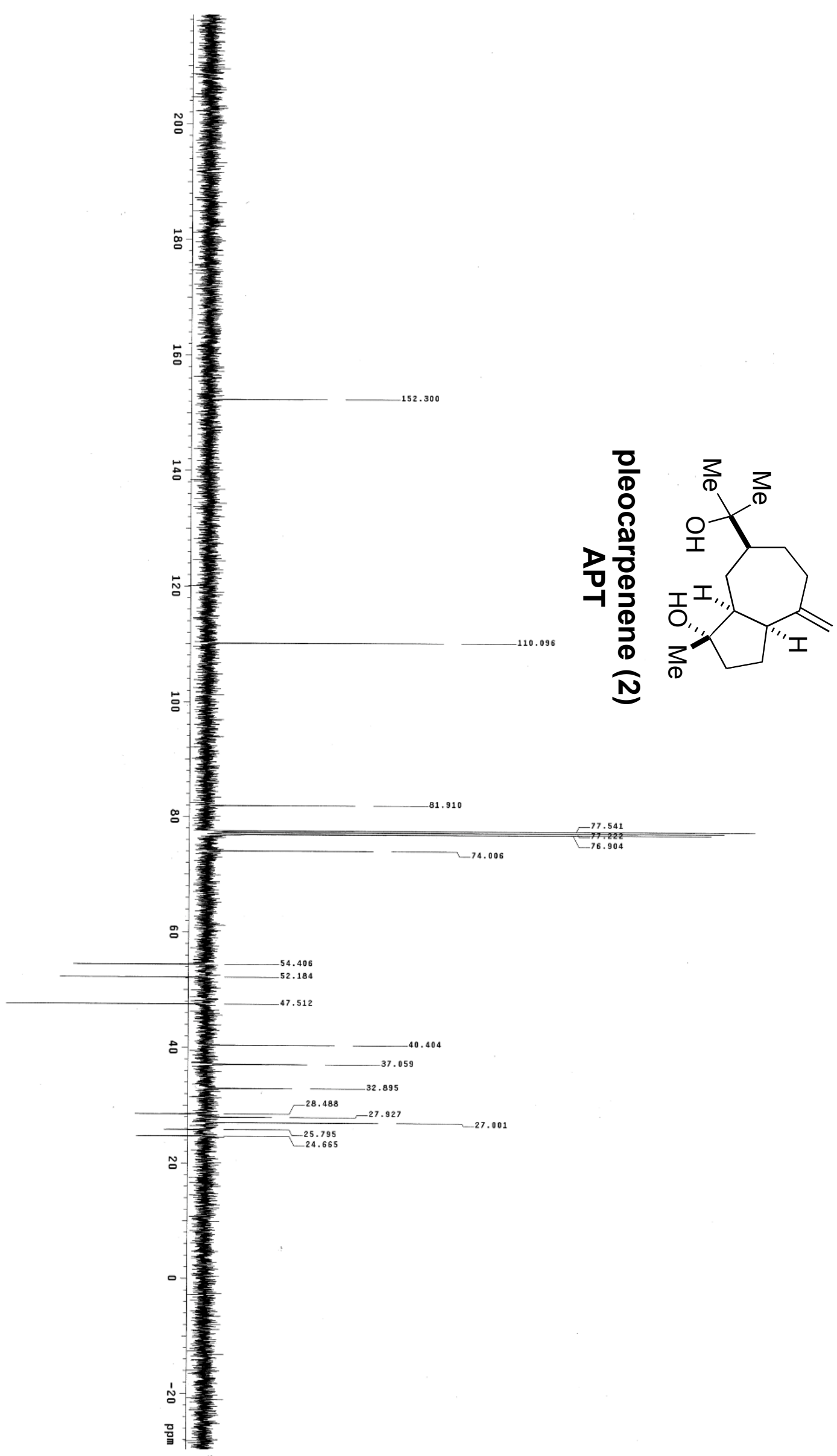



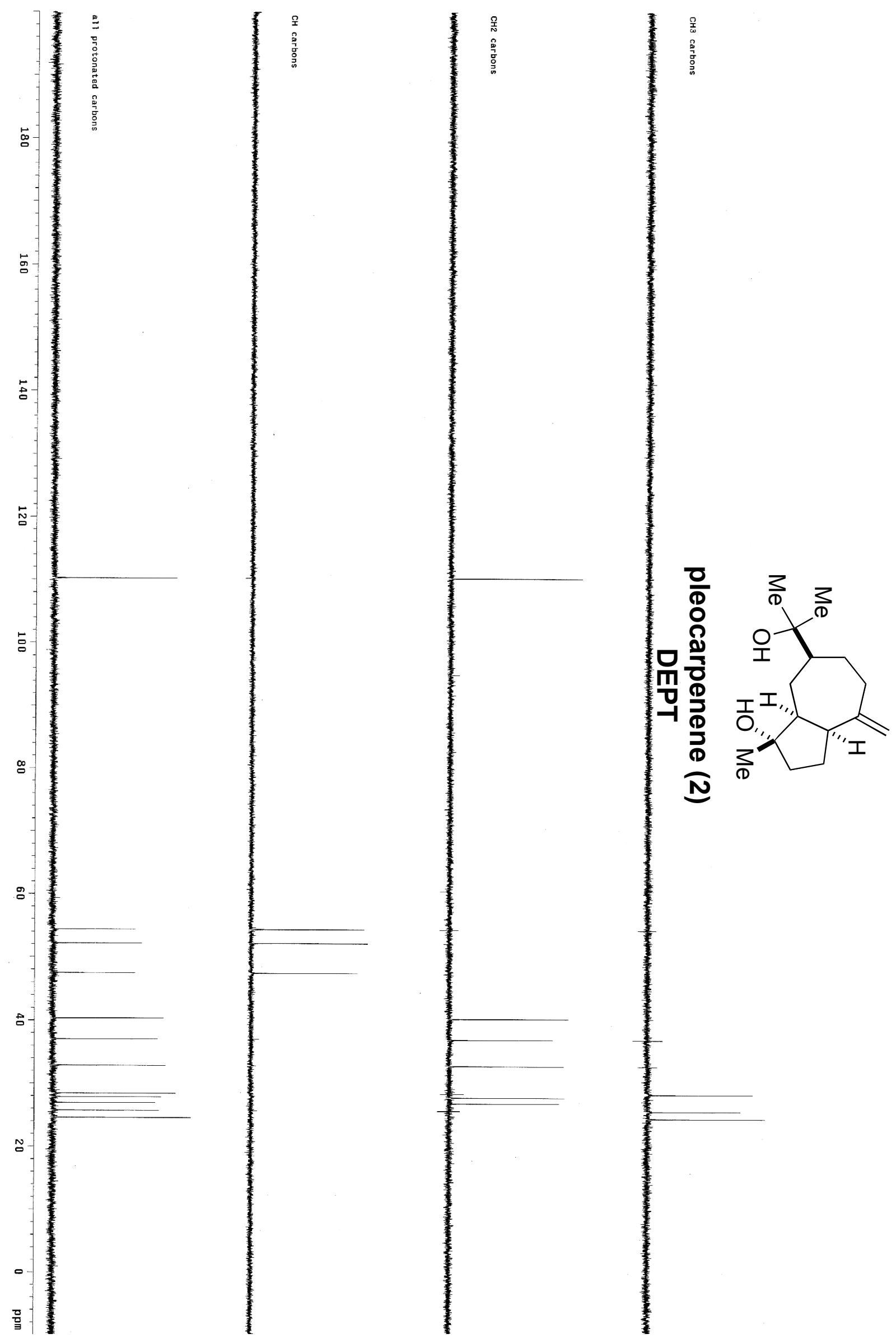


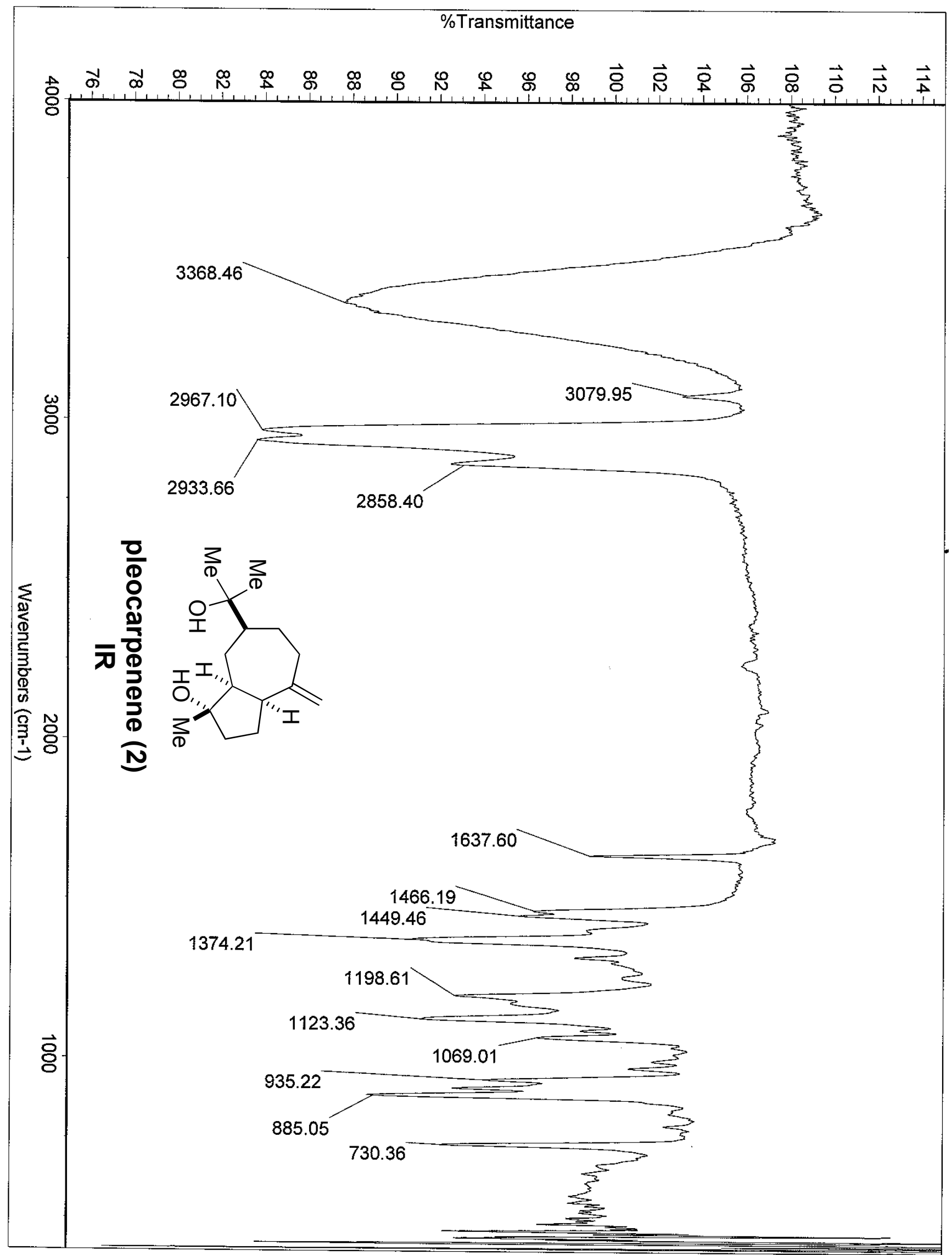


$-S 76-$

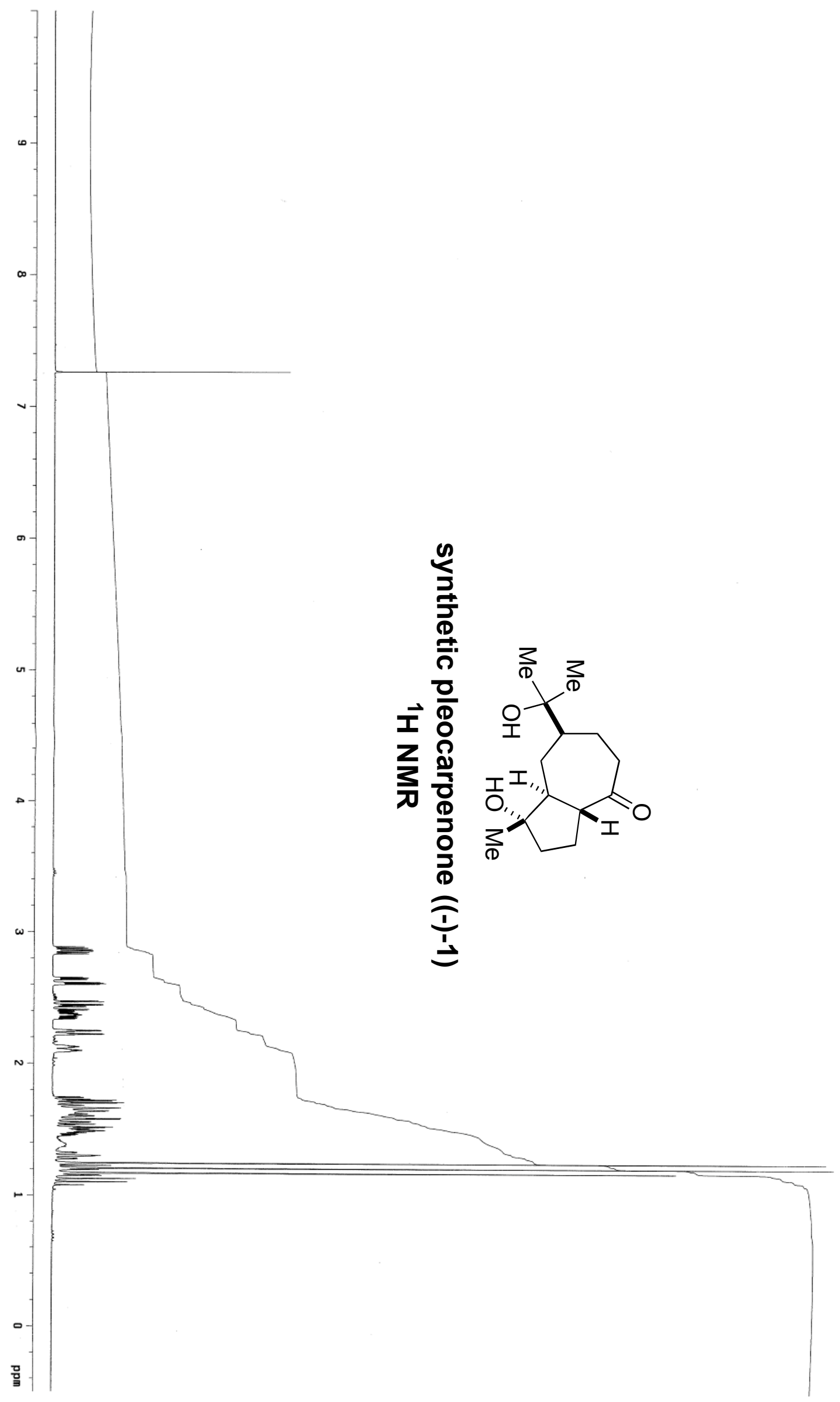




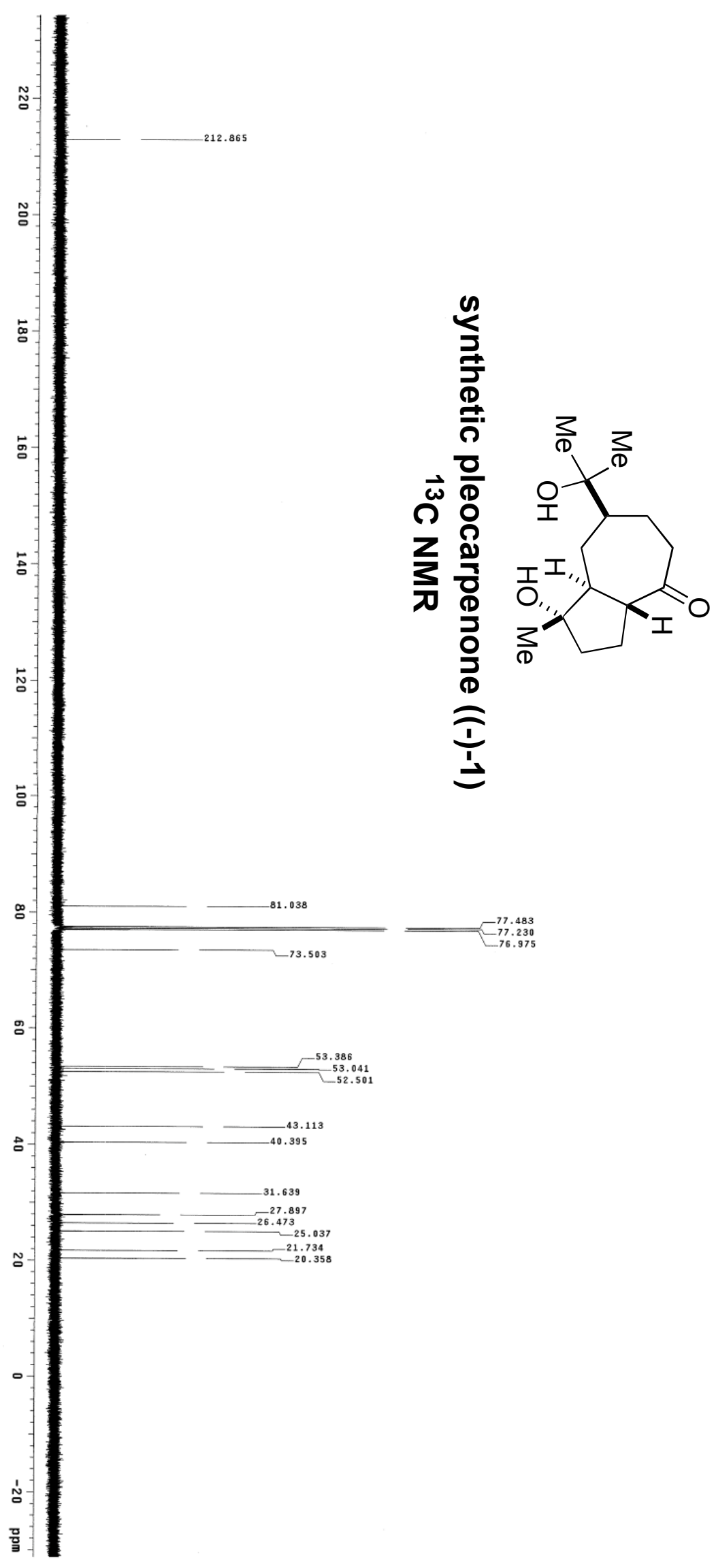




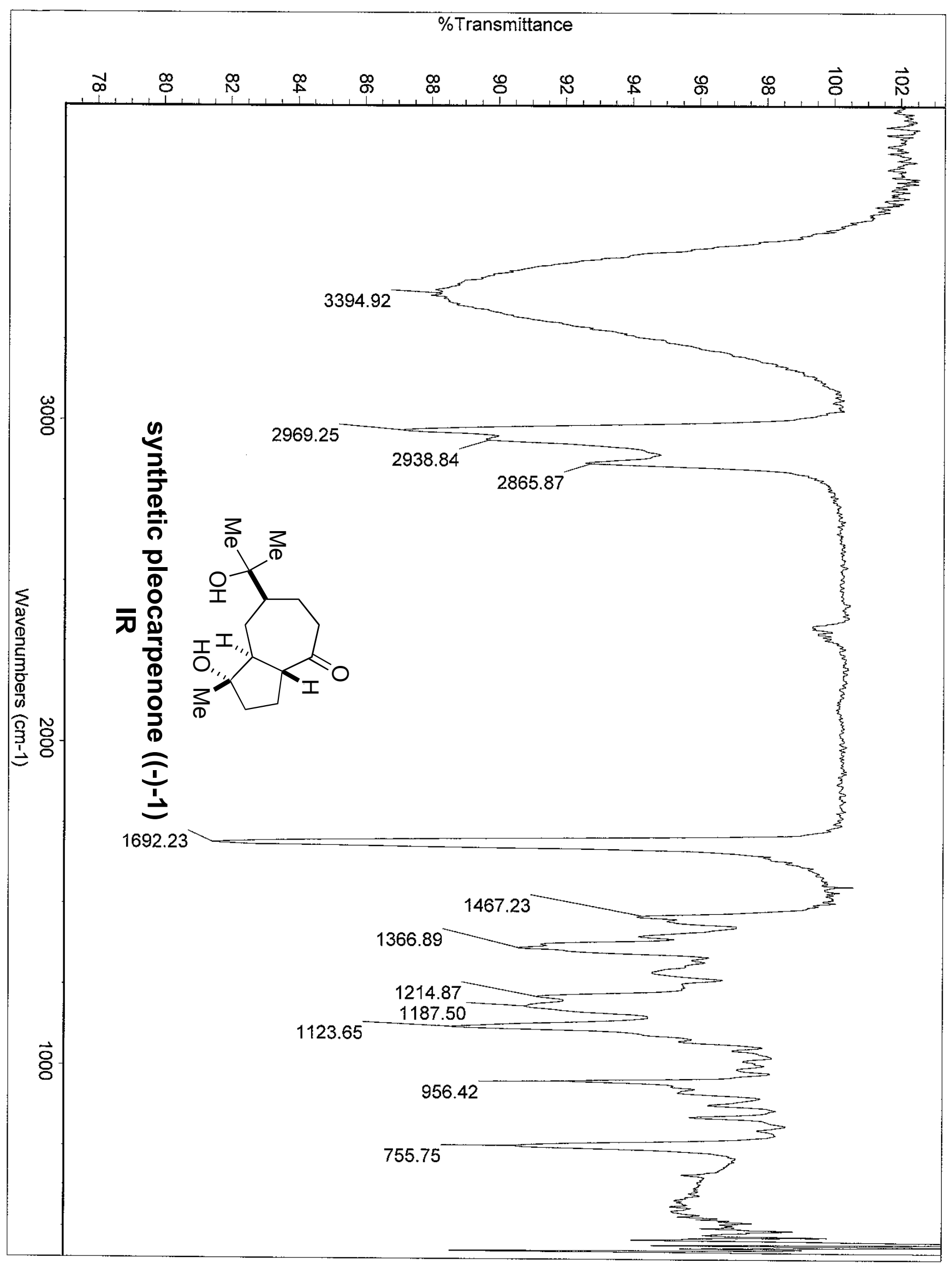


-S 79 -

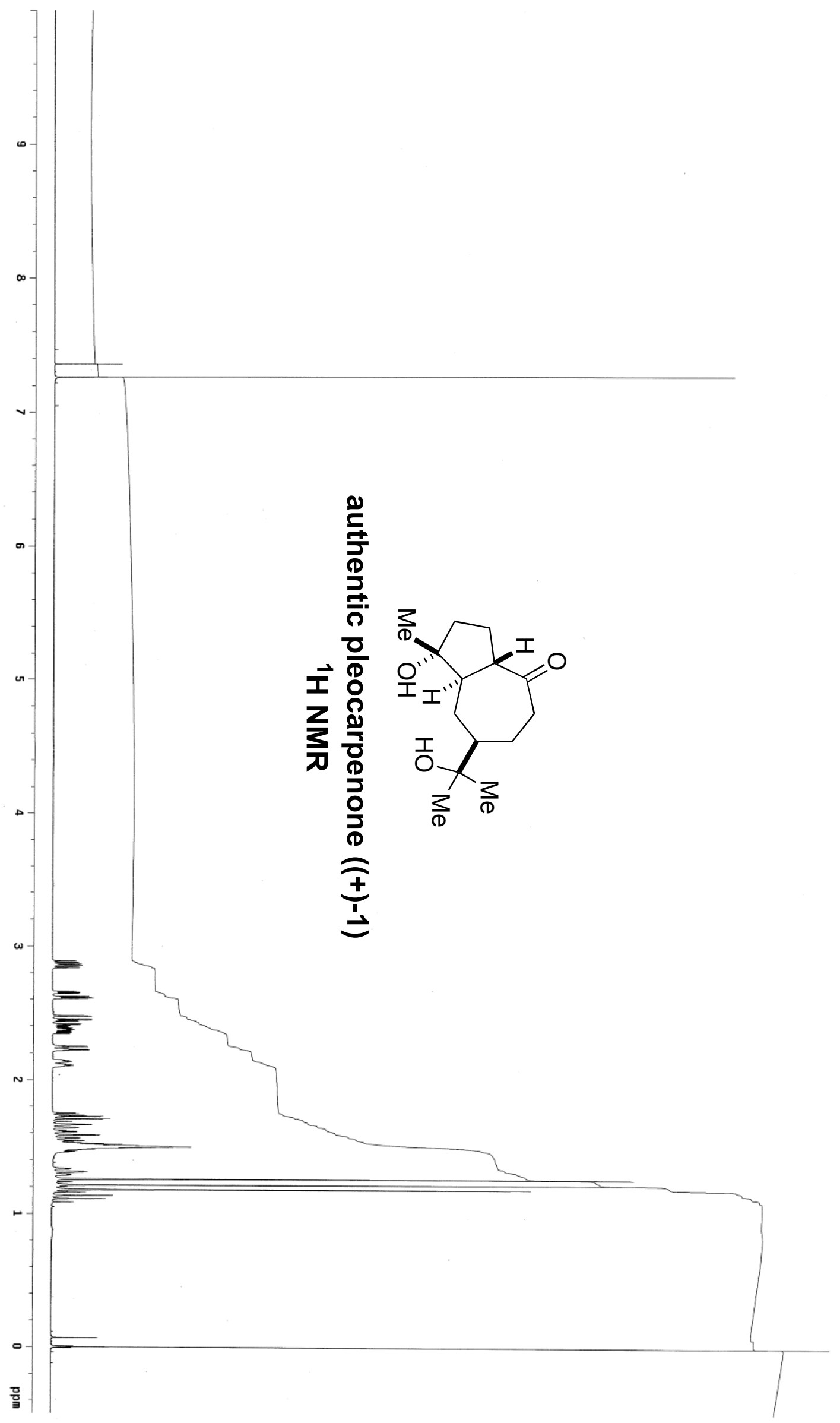




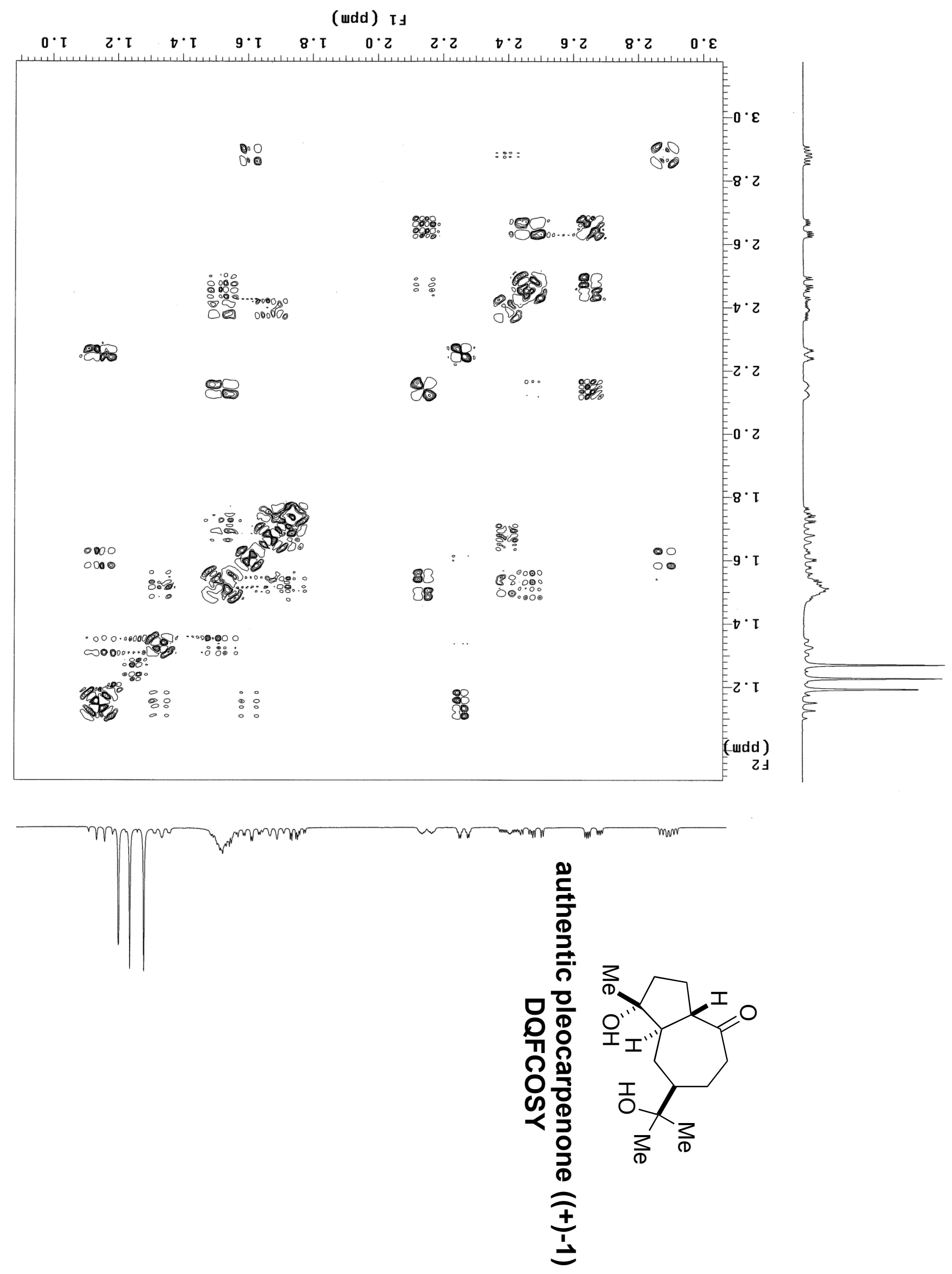


(udd) $I t$
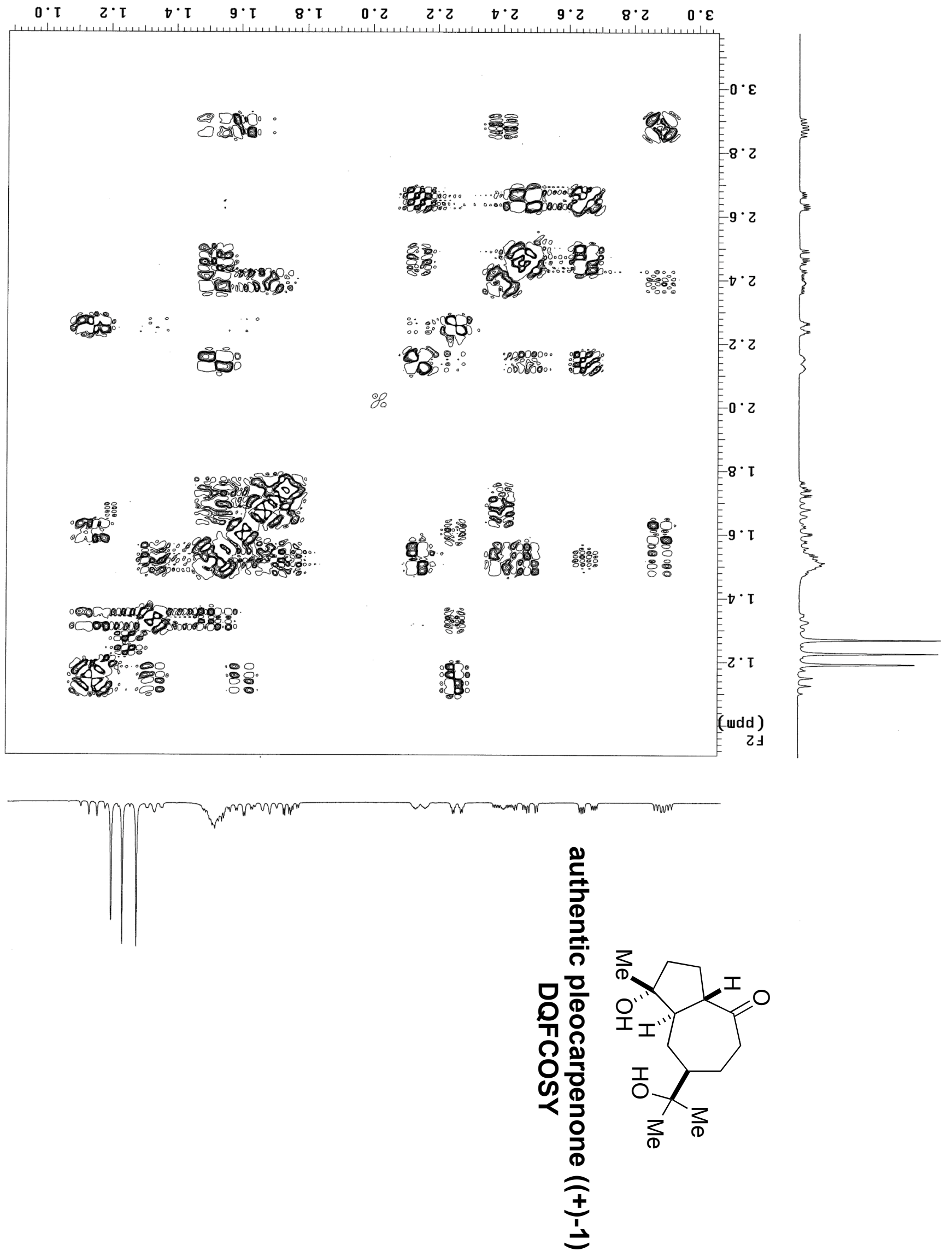
$-S 82-$

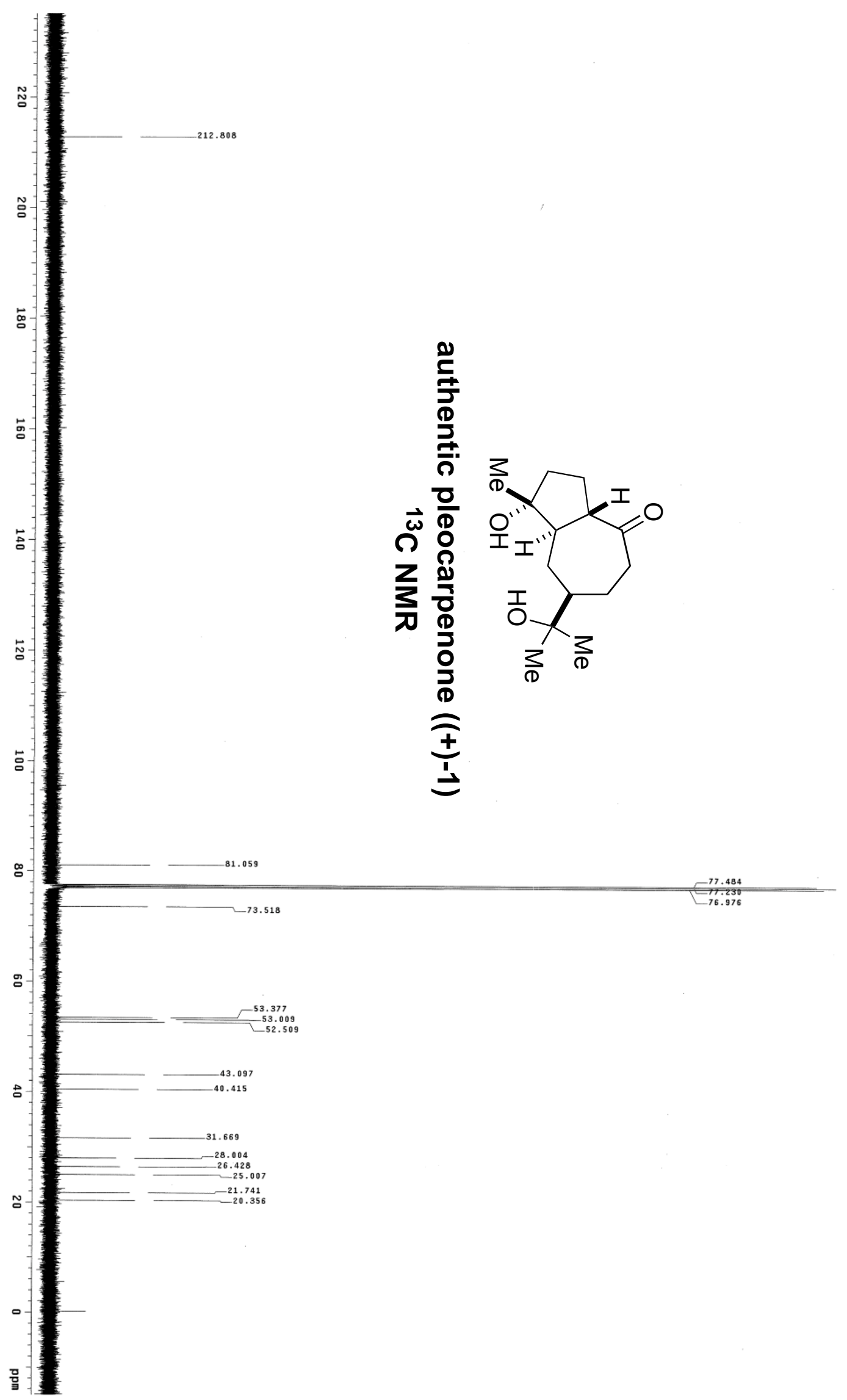


$-S 83-$

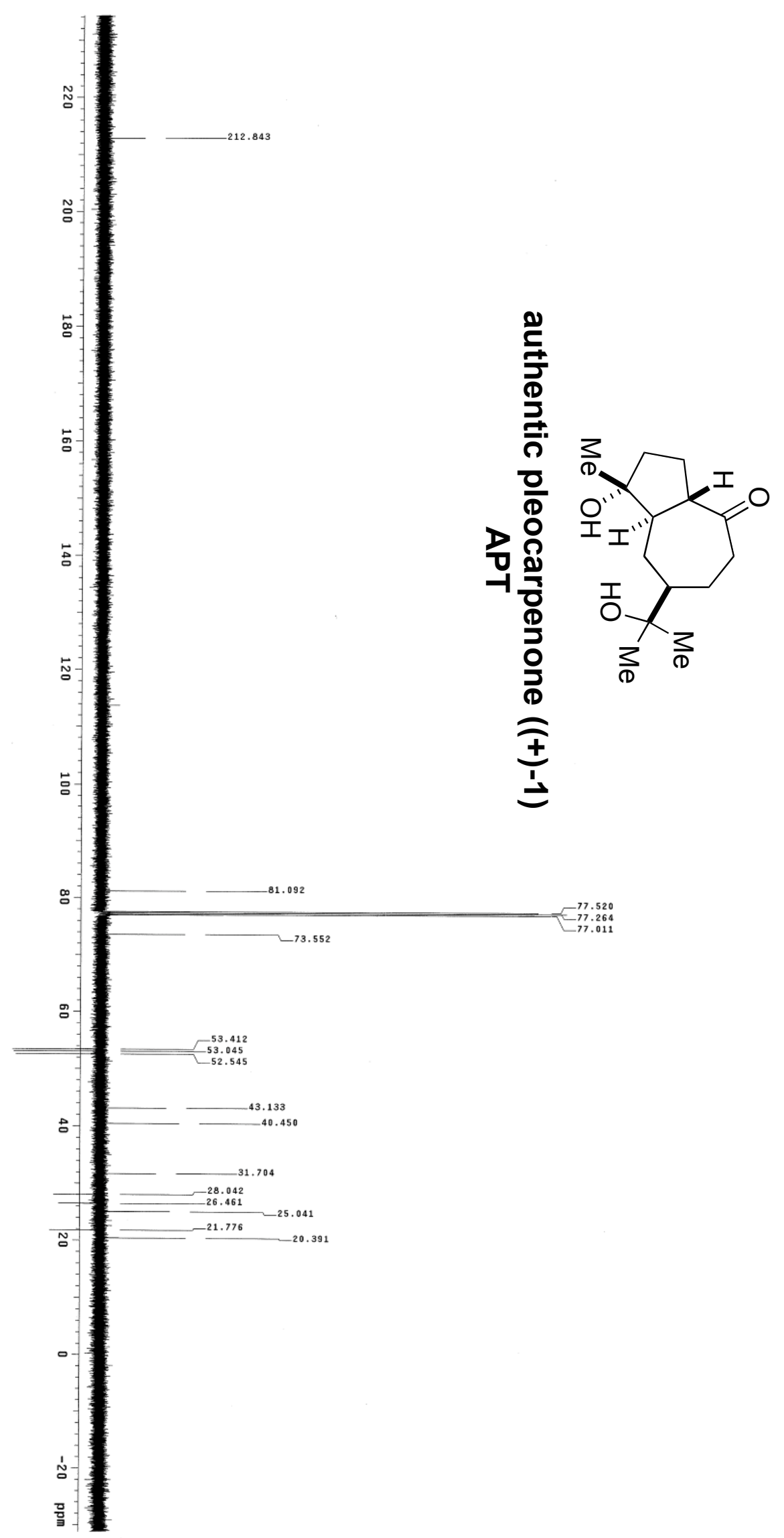


$-S 84-$
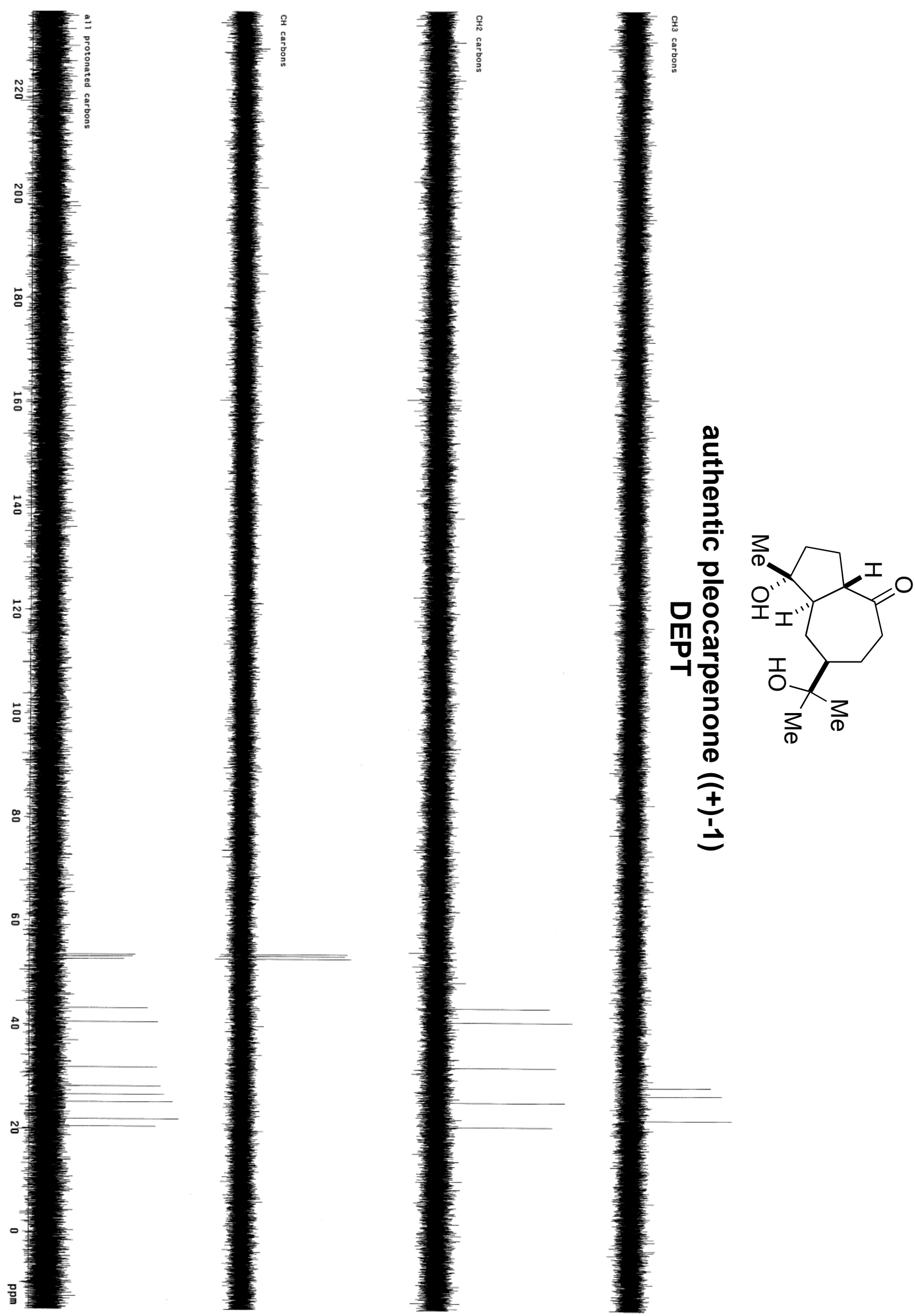


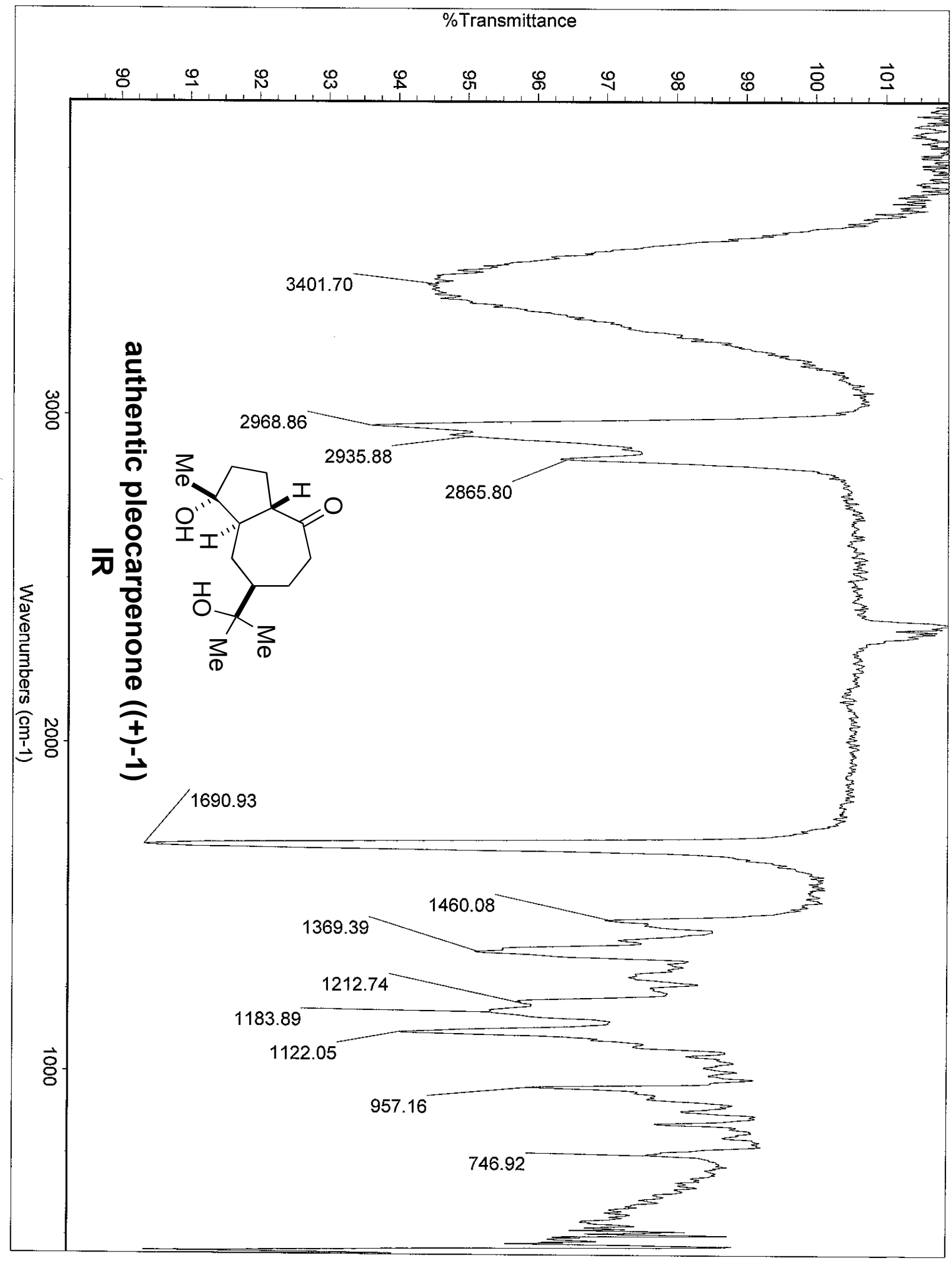

